\title{
"Integration of Smart Antennas with Software Radio"
}

\author{
by \\ “Wei Wang, M.E." \\ A thesis submitted to the \\ Faculty of Graduate Studies and Research \\ in partial fulfillment of the requirements \\ for the degree of \\ "Master of Engineering"

\begin{abstract}
Ottawa-Carleton Institute for Electrical Engineering
Faculty of Engineering

Department of Systems and Computer Engineering

Carleton University
\end{abstract}

“May 28, 2000”
@ copyright
“2000, Wei Wang” 
The undersigned recommend to the Faculty of Graduate Studies and Research acceptance of the thesis

\title{
"Integration of Smart Antennas with Software Radio"
}

\author{
submitted by \\ "Wei Wang, M.E” \\ in partial fulfillment of the requirements \\ for the degree of \\ "Master of Engineering"
}

"Professor S.A. Mahmoud and Professor R.H.M. Hafez", thesis supervisor

"Professor Rafik A. Goubran", Chair, Department of Systems and Computer Engineering

Carleton University

Date “February 28, 2000” 


\section{Abstract}

This thesis investigates some important issues involving the smart antenna array and its integration with software radio technology in TDMA cellular systems.

Third-generation (3G) wireless systems need strategies to further improve performance, increase data rates and at the same time provide flexible and affordable support for multi-services and multi-standards. Software radio technology is promising to provide the required flexibility in radio frequency $(\mathrm{RF})$, intermediate frequency (IF) and baseband signal processing stages. The smart antenna is one of the attractive advanced processing techniques used to greatly improve the system performance. With smart antennas, the capacity can be enhanced by making use of spatial processing, exploiting the spatial directivity of the smart antenna and reducing co-channel interference. This paper address two main points: (i) the capacity gain analysis of the smart antenna in GSM-like TDMA systems, (ii) the software radio architecture design for the TDMA system base station with a smart antenna. The multiple beam smart antenna, also known as switched beam antenna, is used in our analysis. Technologies such as frequency hopping $(\mathrm{FH})$, perfect power control (PC) and discontinuous transmission (DTX) are considered in our study. The performance is analyzed and compared with the sectorization-only application. Analytical results are given and can be easily extended and applied to any other TDMA systems such as IS-136 or next generation systems such as UMTS. One software radio architecture for a base station with smart antenna is proposed and analyzed. In this architecture, the smart antenna algorithms can be dynamically reconfigured according to different environment requirements and the baseband processing can also be dynamically reconfigured according to different standard requirements. In this way, the need for flexibility is satisfied. 


\section{Acknowledgments}

I am sincerely grateful to my thesis supervisors, Professor Samy Mahmoud and Professor R.H.M. Hafez for their guidance, encouragement, motivation and financial support. I am also grateful to Ph.D. student Mohamed Ahmed and Dr. Ian Marsland, for devoting substantial time and energy in discussion on thesis-related research issues and for contributing invaluable ideas.

Thanks to my dear wife, my parents and all family members for their emotional support and encouragement. There are no words to describe what you mean to me. All my accomplishments, past, present, and future, are a direct result of your guidance, your support and your love. 


\section{List of Contents}

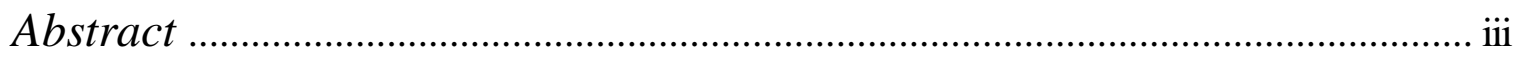

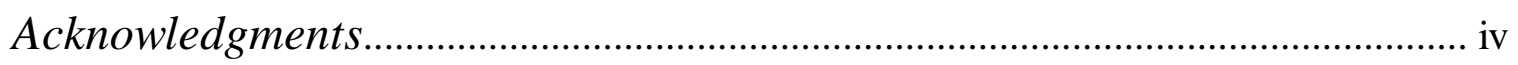

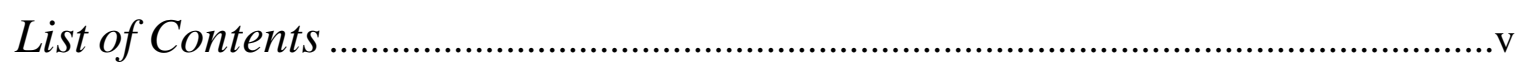

List of Figures ...................................................................................................... vii

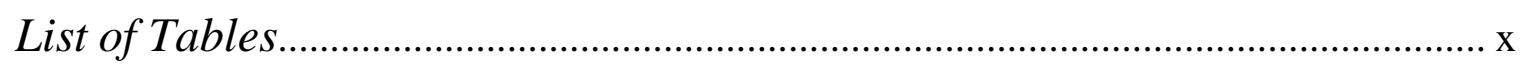

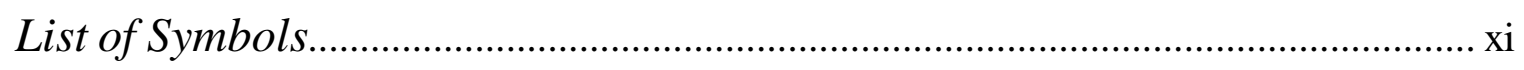

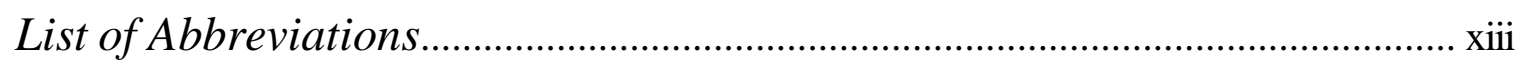

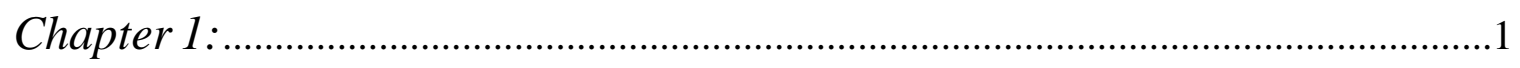

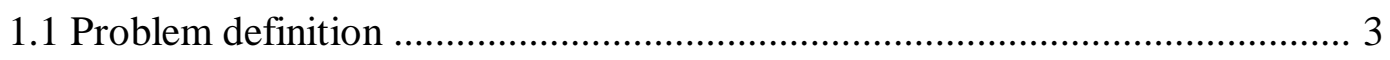

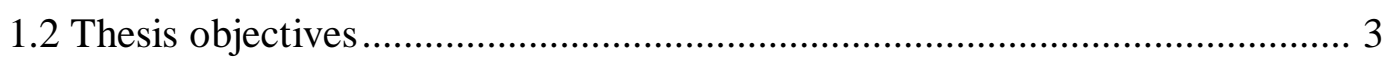

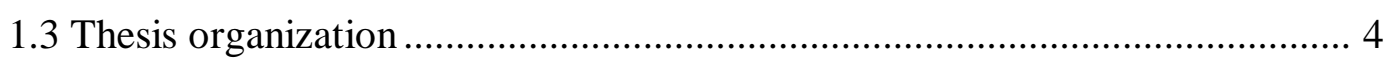

Chapter 2:

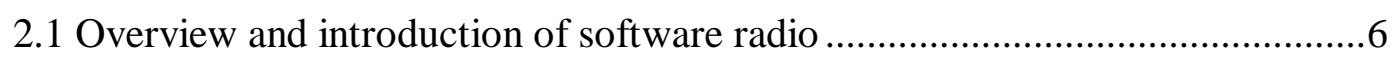

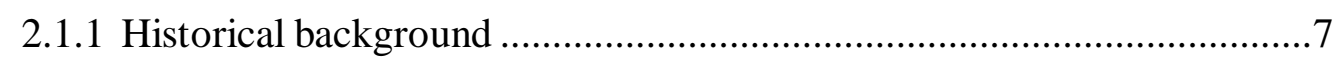

2.1.2 Concept of the ideal software radio.....................................................

2.1.3 Benefits of SWR for multi-standard systems ……...............................

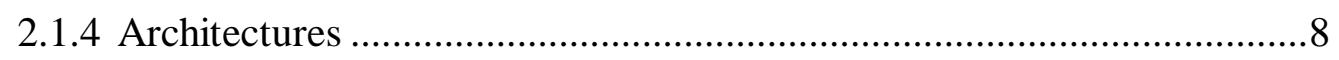

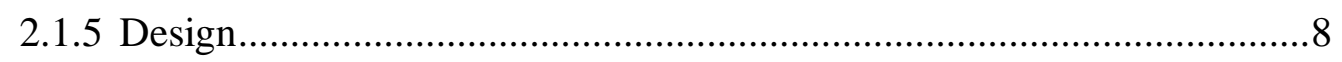

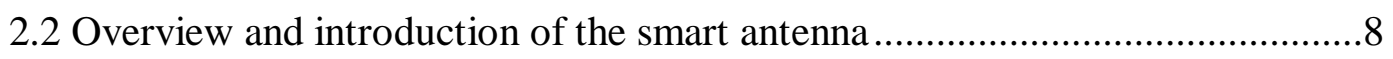

2.2.1 Challenges in wireless communication systems .....................................

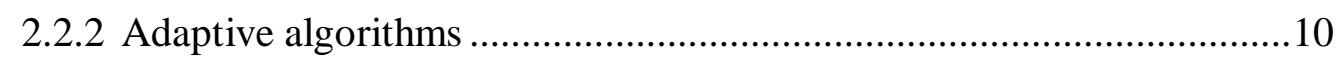

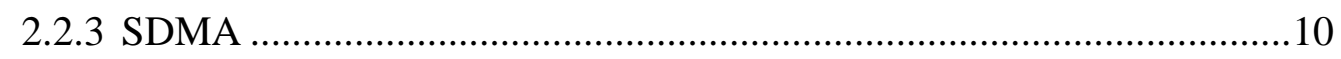

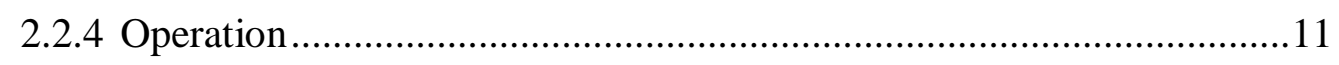

2.3 Synergies between software radio base stations and smart antennas ..............11 


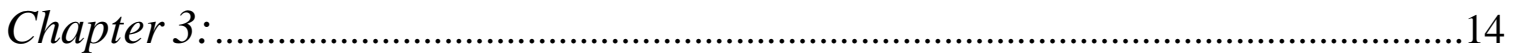

3.1 Introduction

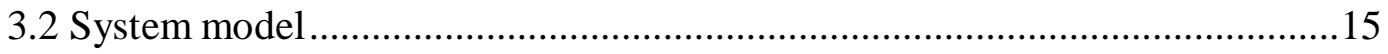

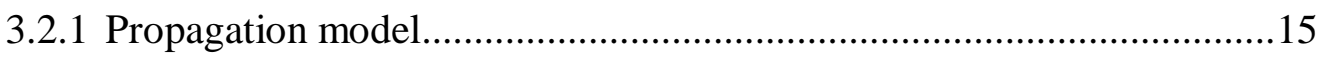

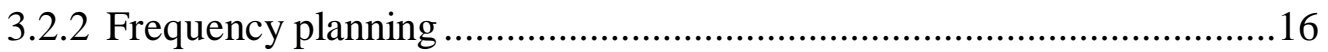

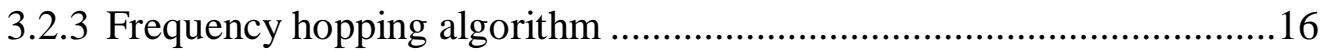

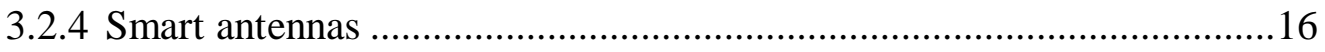

3.3 Analysis and signal-to-interference ratio calculations for the down-link ........18

3.3.1 Switched beam antenna with no power control ....................................23

3.3.2 Multibeam antenna with perfect power control ......................................27

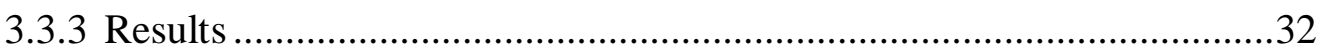

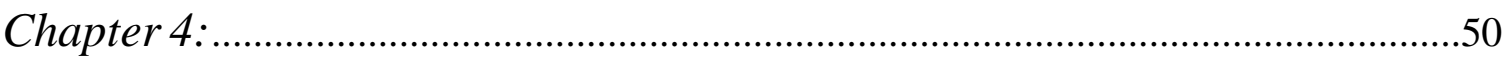

4.1 Baseband processing function review for GSM and IS-54 ............................50

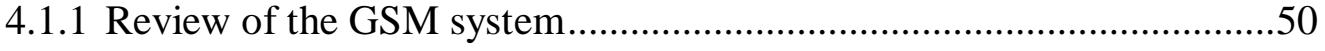

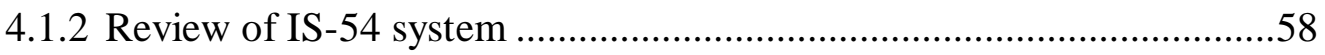

4.2 Baseband software radio architecture for TDMA base station.........................60

4.2.1 Common modulator structure..............................................................60

4.2.1.1 Pi/4-DQPSK modulation scheme …………...........................60

4.2.1.2 Linear approximation of GMSK modulation scheme...............61

4.2.2 One possible common demodulator architecture ……………...............65

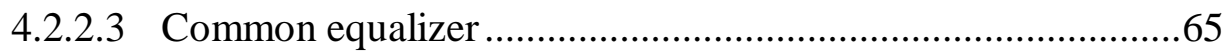

4.3 Parameterized structure for channel coding/interleaving ……………….........68

4.4 SWR architecture for the baseband processing of main function.....................71

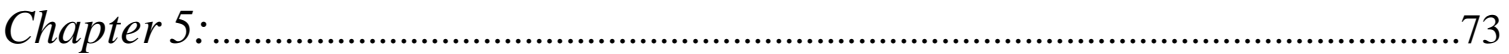

5.1 Smart antenna and beamforming algorithms review .....................................73

5.1.1 Adaptive beam smart antenna ............................................................

5.1.2 Spatial/temporal adaptive beamforming methods ..................................75

5.1.2.1 Space diversity combining ........................................................

5.1.2.2 Space-time diversity combining ………………......................

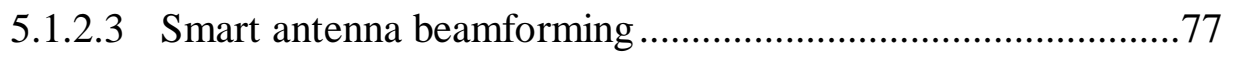

5.2 Beamforming of smart antennas in GSM …………....................................

5.3 Base station software radio architecture evolution .........................................8

5.4 Functional block diagram of the SWR base station with smart antennas........82 


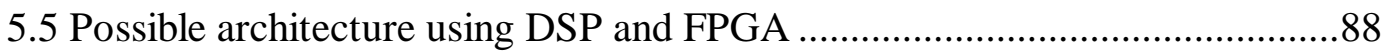

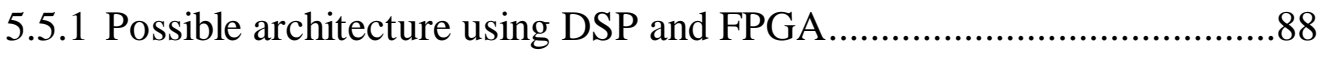

5.5.2 Advantages and limitations ..............................................................91

5.6 Channel assignment algorithm in GSM system with smart antenna ...............91

Chapter 6:

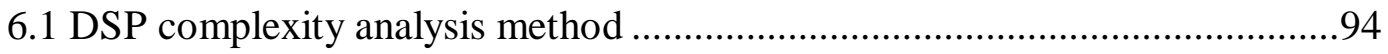

6.2 Partitioning of the channel processing stream ...............................................95

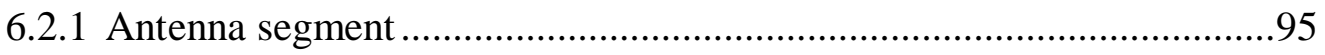

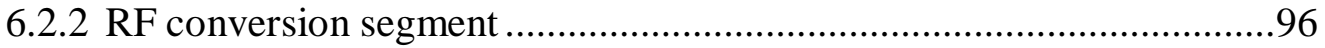

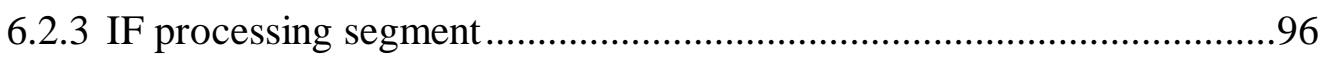

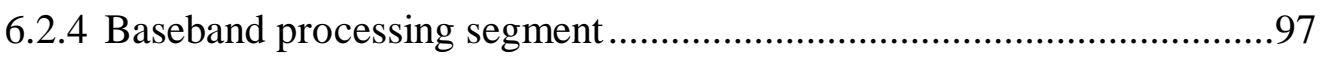

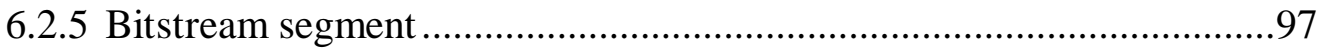

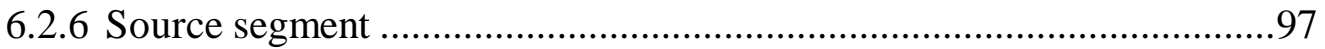

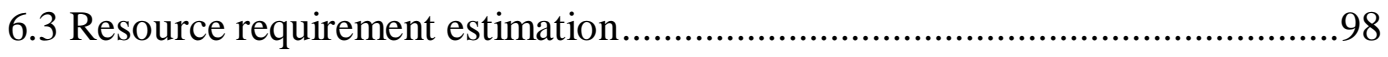

6.3.1 Estimate demand in the context of the canonical data flow ....................98

6.4 Estimating total resource requirements......................................................99

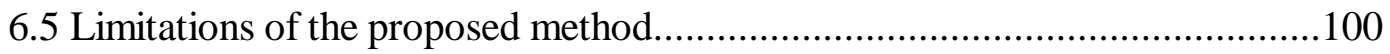

Chapter 7:

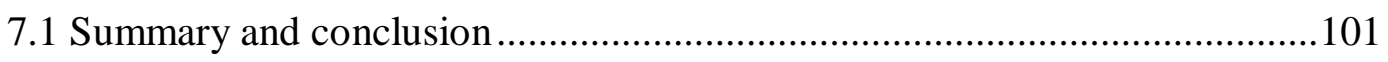

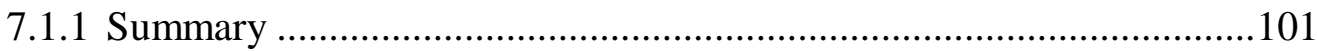

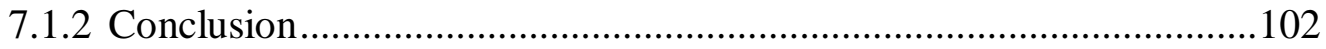

7.2 Recommendations for future research .......................................................103

References: 


\section{List of Figures}

Figure 2.1: Array response of the "smart antenna" .12

Figure 3.1: Flow chart of analysis procedures 19

Figure 3.2: Downlink co-channel interference from the first tier interferers and 5 second tier interferers. .20

Figure 3.3: Down link co-channel interference with frequency hopping. .21

Figure 3.4: Downlink co-channel interference from interference cell $j$ without power control .22

Figure 3.5: Downlink co-channel interference from interference cell $\mathrm{j}$ with perfect power control.. .28

Figure 3.6: CDF of CIR for a cell at 20\% Load Factor ...............................................

Figure 3.7: CDF of CIR for a cell at 40\% Load Factor ...............................................36

Figure 3.8: CDF of CIR for a cell at 60\% Load Factor ..................................................37

Figure 3.9: $\quad$ CDF of CIR for 3-sector antenna without switch beam (no power control)

Figure 3.10: $\mathrm{CDF}$ of CIR for 3-sector antenna with switch beam (no power control) ..39

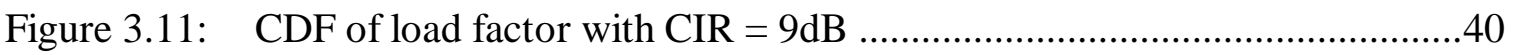

Figure 3.12: CDF of CIR for a cell at 20\% Load Factor ...............................................41

Figure 3.13: CDF of CIR for a cell at 20\% Load Factor ................................................

Figure 3.14: CDF of CIR for a cell at 40\% Load Factor ...............................................43

Figure 3.15: CDF of CIR for a cell at 40\% Load Factor ...............................................4

Figure 3.16: CDF of CIR for a cell at 60\% Load Factor .............................................45

Figure 3.17: CDF of CIR for a cell at 60\% Load Factor .............................................46

Figure 3.18: $\quad$ CDF of CIR for 3 sector antenna without switch beam under power control

Figure 3.19: $\quad$ CDF of CIR for 3 sector antenna with switch beam under power control 48 Figure 3.20: Outage probability as function of load factor with $\mathrm{CIF}=9 \mathrm{~dB}$

Figure 4.1: A block diagram of transmitter and receiver (channel decoding) for a GSM base station.

Figure 4.2: $\quad$ Physical structure of hyperframe, superframe, multiframe, frame, and time

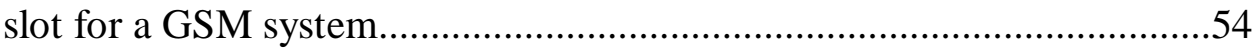

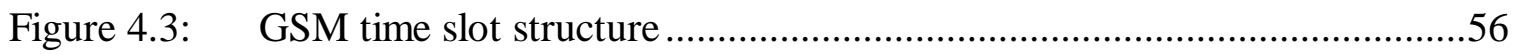

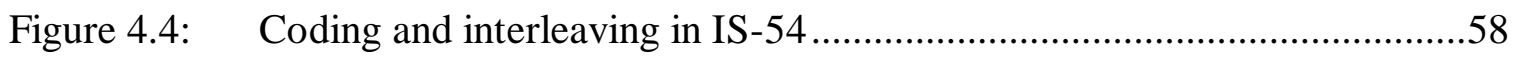




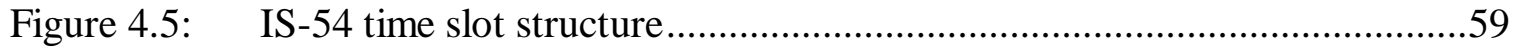

Figure 4.6: One Common I/Q-modulator with different system parameters ...............64

Figure 4.7: One common receiver structure for GSM and IS-54 …..........................67

Figure 4.8: Parameterized structure for Convolutional Channel Coding .....................69

Figure 4.9: Parameterized structure for interleaving ...............................................

Figure 4.10: Parameterized architecture for baseband processing ................................71

Figure 4.11: Parameterized functional structure for baseband processing with smart an-

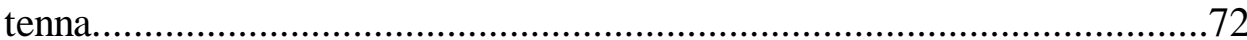

Figure 5.1: An adaptive array system with space diversity combiner..........................74

Figure 5.2: Possible software radio architecture evolution for base station...................81

Figure 5.3: Functional block diagram of the software radio for a multimode base station

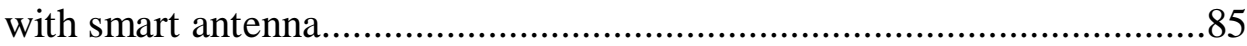

Figure 5.4: Diagram of beamformer for each channel ..............................................

Figure 5.5: Diagram of possible weights generator for the beam former ....................87

Figure 5.6: General functional architecture for software radio base station with smart

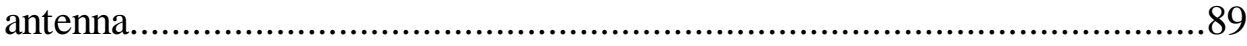

Figure 5.7: Functional architecture for software radio base station with smart antenna

Figure 5.8: Flow chart of the channel assignment algorithm with smart antenna........93

Figure 6.1: The canonical software radio function architecture with smart antenna ap-

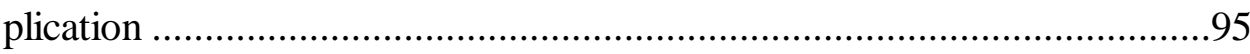




\section{List of Tables}

Table 3.1: $\quad$ Outage Probability $(\mathrm{CDF}(\mathrm{CIR}=9 \mathrm{~dB}))$ at different loading factor values.

Table 3.2: Maximum loading factor (percentage) with various outage probability requirements ...................................................................................... 33

Table 4.1: $\quad$ Parameters relating to the proposed common viterbi equalizer.................. 66

Table 4.2: $\quad$ The main channel coding parameter for GSM and IS-54 ......................68

Table 4.3: $\quad$ The main interleaving parameter for GSM and IS-54 .........................68

Table 5.1: $\quad$ Smart antenna processing algorithms ................................................ 78

Table 5.2: $\quad$ Smart antenna beamforming algorithms ........................................... 79

Table 5.3: $\quad$ Issues related to smart antenna integration ............................................79

Table 6.1: $\quad$ Critical parameters for demand estimation ........................................99

Table 6.2: Approximation processing demand estimation: GSM with smart anten-

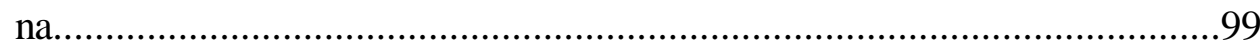




\section{List of Symbols}

$\vec{a}\left(\theta_{j n}\right) \quad$ Array response to the multipath signal arrive from direction $\theta_{j n}$

$A_{\text {cell }}$ Cell area

$A_{j}$

Bernoulli random variable representing voice activity of jth interference MS

BT Time bandwidth product (filter bandwidth times bit period)

$C \quad$ Carrier power

$\mathrm{CH}_{\text {cell }} \quad$ Number of channels per cell

$C_{k} \quad$ A serial amplitude modulated pulse [61]

$d \quad$ Distance between BS and MS

$D_{j} \quad$ Distance from BS in cell 0 to interference BS in cell $\mathrm{j}$

$E_{\text {user }} \quad$ Average traffic in Erlangs per user

$f_{\text {carrier }} \quad$ Carrier frequency

$f_{s} \quad$ Sampling frequency

F $\quad$ Fading parameter

$g(\theta) \quad$ Directive gain of the smart antenna

$g(t) \quad$ Pulse former, a square root raised filter with roll off factor $\alpha$

$G_{j} \quad$ Link gain between the jth transmitter and BS with smart antenna

$h \quad$ Modulation index

$h_{\text {Gauss }}(t) \quad$ Gaussian impulse with time bandwidth product BT

$I_{j} \quad$ Interference power from interference cell $\mathrm{j}$

$I_{n} \quad$ Complex symbol in $\mathrm{p}_{\mathrm{i}} / 4-\mathrm{DQPSK}$ modulation

$l_{j}\left(r_{0}, \theta_{0}\right) \quad$ Distance from MS in cell 0 to interference BS in cell $\mathrm{j}$

$K \quad$ Number of co-channel transmitter

L Length of FIR filter

$M \quad$ Element number of smart antenna array

$m W \quad$ miliwatts

$\mathrm{n} \quad$ Path loss exponent

$N \quad$ Maximum possible multipath

$N_{0} \quad$ Thermal noise power 


\begin{tabular}{|c|c|}
\hline$N_{b}$ & Beam number \\
\hline$N_{\text {int }}$ & Number of co-channel interference cell \\
\hline$P_{a}$ & Available power from the source \\
\hline$P_{r}$ & Received power \\
\hline$P_{t}$ & Transmit power \\
\hline$P_{\text {out }}$ & Outage probability \\
\hline$r_{0}$ & Distance from MS to BS in cell 0 \\
\hline$r_{j}$ & Distance from interference MS to BS in cell $\mathrm{j}$ \\
\hline$R$ & Cell radium \\
\hline$R_{b}$ & Bit rate \\
\hline$R_{G S M}$ & Bit rate for GSM system \\
\hline$R_{T D M A}$ & Bit rate for TDMA system \\
\hline$S N R_{r e q}$ & Required signal-to-noise ratio \\
\hline$s_{j}(k)$ & Transmit signal from each of K co-channel transmitter \\
\hline$S$ & Constant received power at MS if power control applied \\
\hline$\alpha_{j n}$ & Nth path fading coefficient for jth user \\
\hline$\gamma$ & CINR threshold \\
\hline$\lambda$ & Normal random variable corresponding to shadowing \\
\hline$\lambda_{j}$ & Shadowing parameter of path from jth interferer BS to MS in cell 0 \\
\hline$\lambda_{j}^{\prime}$ & Shadowing parameter of path from 0 th $\mathrm{BS}$ to $\mathrm{MS}$ in cell 0 \\
\hline$\lambda_{j j}$ & Shadowing parameter of path from $\mathrm{jth} B S$ to MS in cell $\mathrm{j}$ \\
\hline$\theta_{0}$ & Angle of MS in Cell 0 \\
\hline$\theta_{b 0}$ & Angle connected from BS in cell 0 to MS \& interfer. BS in cell $\mathrm{j}$ \\
\hline$\theta_{0 j}$ & Center angle of the corresponding beam containing MS in cell $\mathrm{j}$ \\
\hline$\theta_{j 0}$ & Angle of connection from BS in cell $\mathrm{j}$ to MS in cell 0 ; \\
\hline$\theta_{j j}$ & Angle of MS in interference cell $\mathrm{j}$ \\
\hline$\theta_{b j}$ & Center angle of the corresponding beam containing MS in cell $\mathrm{j}$ \\
\hline$\sigma$ & Standard deviation of Log-normal distribution \\
\hline$\tau_{\max }$ & Maximum path delay \\
\hline$\vec{x}(k)$ & Total received multipath signal vector from K cochannel transmit- \\
\hline
\end{tabular}


$\vec{\omega}_{i} \quad$ Weighting vector of smart antenna

\section{List of Abbreviations}

3G Third Generation

A/D Analog-to-Digital

ACI Adjacent Channel Interference

ADC Analog-to-Digital Converter

AGC Automatic Gain Control

AMPS Advanced Mobile Phone System

API Application Programmer's Interface

ASIC Application-Specific Integrated Circuit

BCCH Broadcast Channel

BF Baseband Frequency

BPF Band Pass Filter

BS Base Station

BW Bandwidth

$\mathrm{CCCH}$ Common Control Channel

CCI Co-Channel Interference

CDF Cumulative Density Function

CDMA Code Division Multiple Access

CH1 Channel 1

$\mathrm{CH} 2$ Channel 2

C/I Carrier-to-Interferer Ratio

CIR Carrier-to-Interferer Ratio

CINR Carrier to Noise and Interference Ration

CMA Constant Modulus Algorithm

C/N Carrier-to-Interference

CRC Cyclic Redundancy Check

$\mathrm{dB} \quad$ Decibels

D/A Digital-to-Analog

DAC Digital-to-Analog Converter

D-AMPS Digital-Advanced Mobile Phone System

DCS-1800 North American upband GSM standard

DCO Digitally Controlled Oscillator

Demod. Demodulator

DECT Digital European Cordless Telephone 
DFT Discrete Fourier Transform

DOA Direction of Arrival

DOB DOA based beamforming

DQPSK Differentially Encoded Quaternary-Phase-Shift-Keying

DSP Digital Signal Processing

DTX Discontinuous Transmission

DTXF Discontinuous Transmission Factor

FDMA Frequency Division Multiple Access

FH Frequency Hopping

FR Full Rate

FPGA Field-Programmable Gate Array

GHz Giga Herz

GMSK Gaussian Minimum Shift Keyed

GPS Global Positioning System

GSM Groupe Speciale Mobile

Gen. Generator

IF Intermediate Frequency

IM Intermodulation

IS-54 American TDMA cellular radio interface standard

IS-95 Wireless CDMA radio interface standard

ISI Inter-Symbol Interference

ITU International Telecommunication Union

KB Kilobytes

$\mathrm{kHz} \quad$ Kilo Herz

LF Loading Factor

LMS Least Means Square

LOS Line of Sight

LPF Low Pass Filter

M Element Number of Smart Antenna

Meg Megabytes

MEM Maximum Entropy Method

MB Megabytes

$\mathrm{MHz} \quad$ Mega Herz

MLSE Maximum Likelihood Sequence Estimation

MMSE Minimum Mean Square Error

MOPS Millions of Operations Per Second

MS Mobile Station

MSPS Mega Samples Per Second 


\begin{tabular}{ll} 
MUSIC & Multiple Signal Classification \\
MVDR & Minimum Variance Distortionless Response \\
NF & Noise Figure \\
NRZ & None Return Zero \\
OS & Operating System \\
PA & Power Amplifier \\
PC & Power Control \\
PCS & Personal Communication System \\
PCS-1900 & European upband GSM standard \\
PLL & Phase Locked Loop \\
QAM & Quadrature Amplitude Modulator \\
rms & root mean square \\
RACH & Random Access Channel \\
REPLTP & Regular Pulse Excitation with Long Term Prediction \\
RF & Radio Frequency \\
RLS & Recursive least-square \\
Rx & Receiver \\
sb & Switched Beam \\
SDCCH & Stand-alone Dedicated Control Channel \\
SACCH & Slow Associated Control Channel \\
SDK & Software Development Kit \\
SDMA & Space Division Multiple Access \\
SFDR & Spurious-Free Dynamic Range \\
S/N & Signal-to-Noise \\
SNR & Signal-to-Noise Ratio \\
SIR & Signal-to-Interference Ratio \\
SQNR & Signal-to-Quantization Noise Ratio \\
SSBF & Signal Structure Based Beamforming \\
SWR & Software Radio \\
TCH & Traffic Channel \\
TDMA & Time Division Multiple Access \\
Tx & Transmitter \\
TRB & Temporal Reference Beamforming \\
UMTS & Universal Mobile Telecommunications System \\
W-CDMA & Wideband CDMA \\
WPA & Wideband Power Amplifier \\
\hline SDA &
\end{tabular}




\section{Chapter 1}

\section{Introduction}

Recent research interests in the field of personal mobile radio communications have been moving to the next generation cellular system to meet the following two main growing demands: (i) higher capacity, quality and variable transmission speed of multimedia information [11]-[16], (ii) higher flexibility support for multi-services and multi-standards.

The smart antenna and software radio technology are key solutions to satisfy these demands. The smart antenna is used to greatly enhance system capacity and performance by reducing co-channel interference, exploiting the spatial directivity of the antenna and making use of spatial processing. Software radio will provide the required flexibility in radio frequency (RF), intermediate frequency (IF) and baseband signal processing stages of the system.

Before the smart antenna system, several traditional methods were used to enhance the coverage, quality and capacity of the cellular network. Examples are cell splitting and sectorization.

Cell splitting, a method to split a cell into smaller cells, is efficient, but time-consuming and expensive. It requires building new cellular base stations, which is difficult in many high traffic density areas, where cell sizes are already about as small as can be accommodated.

Sectorization, a method to split one cell into several sectors, is another choice and is widely used to increase capacity. Unfortunately, the trunking efficiency degrades as a 
trade-off and in many cellular markets, sectorization has already been utilized to its practical limit.

The smart antenna is an economical approach. It can be used in the existing cell sites and eliminates the cost of deploying new sites. At the base station, an adaptive control algorithm is used to keep a narrow beam pointed in the direction of each subscriber served by the cell. The effective gain and carrier-to-interference ratio can be dramatically improved and hence the system capacity is increased. The application of smart antennas in wireless mobile communication systems has been studied thoroughly in [15]-[23].

Software radio architecture was originally developed for military applications. Its initial concept came from the idea to implement the fundamental processes, modulation, equalization and demodulation in software [1]-[7]. It has become more and more attractive because:

Software radio has been seen as a potential solution to support a multi-standard cellular system. Operation of multiple standards simultaneously on a single base station would allow a flexible network evolution under operator control, thereby maximizing network capacity and operator flexibility.

It also provides the flexibility for some advanced services integration in the existing base stations. One good example is the smart antenna. Software radio architecture makes it possible to integrate it into the existing base stations efficiently.

In addition, it is now becoming economically viable in commercial products because of the rapid advance of DSP and FPGA technology.

In this thesis, we will study the software radio and its integration with an adaptive antenna application in a base station. Capacity enhancement using smart antenna will be examined and the software radio architecture of the base station will be given to provide the flexibility for smart antenna integration and multi-standard support. 


\subsection{Problem definition}

Current personal mobile telecommunication networks have entered a new era. Demands are increased for systems supporting multi-standard and offering higher capacity and higher quality for multimedia traffic.

The evolution and proliferation of wireless standards introduce the risk of incompatibilities between systems and increase substantially the manufacturing and operational costs.

There are several challenging problems, such as how to design a common platform to support the multi-standards network and how to implement value-added service on the existing cellular network efficiently and economically.

Software-defined radio is needed to overcome these risks by allowing a greater range of services and applications to be supported and introducing a high degree of adaptability and flexibility in the configuration of the wireless network. The smart antenna's implementation in software radio architectures might serve as a good example.

Using software radio techniques, a common hardware platform may provide all of the current base station functions and yet retain enough flexibility to meet future requirements through alternative software application programs.

\subsection{Thesis objectives}

In this thesis, we will review the existing TDMA network protocols (GSM, IS-54), analyze the capacity enhancement in TDMA cellular systems (GSM as an example) with smart antenna application in the base station. In addition, we propose a baseband base station software radio architecture, which can perform all the baseband functions of the base station in a common hardware platform and support several air standards simultaneously 
and on which a smart antenna may mount (this permits easy re-configurability of the base station radio interface). Finally, we investigate one possible method for complexity analysis of ideal software radio architecture implementation.

The objectives of this thesis are to:

- Investigate the concepts for software radio and smart antennas and the potential application synergy in future;

- Analyze the downlink capacity enhancement using multibeam smart antennas in the base station of a TDMA system such as GSM;

- Propose a parameterized baseband receiver design method for TDMA multistandard software radio base station receiver architecture;

- Propose a software radio base station receiver architecture with smart antenna application;

\subsection{Thesis organization}

This thesis is organized as follows:

Chapter 1 introduces the research background of the two key solutions, the smart antenna and software radio.

Chapter 2 provides a review of the concepts and brief survey of related work in smart antennas and software radio.

In Chapter 3, downlink capacity is examined and analyzed for a frequency hopping TDMA system (GSM as an example) with multibeam smart antenna application. 
Chapter 4 presents a parameterized design method for TDMA multi-standard software radio base station receiver architecture.

In Chapter 5, a software radio base station architecture with smart antenna application is presented and analyzed. We will see that the smart antenna can be implemented in the base station, on top of the baseband architecture proposed in Chapter 4.

Chapter 6 describes one possible methodology for complexity analysis of software radio architecture.

The thesis summary, conclusion and suggestions for further research are included in Chapter 7. 


\section{Software Radio and Smart Antenna}

\subsection{Overview and introduction of software radio}

During the past five years, the software radio has emerged from military research to become a cornerstone of next generation strategies for affordable high-performance regional and global communications.

The increasing demands for new features and applications, such as smart antenna system, over mobile radio multimedia communication systems will require a major shift from existing network-centered architectures towards flexible, user-defined wireless communication services. Furthermore, the existence of many non-compatible air interface standards in second-generation wireless systems and the move to a new standard for nextgeneration systems, such as UMTS, will require the development of a common platform to support inter-working among these air interface standards. Such a platform must permit easy modifications, in order to allow for customizing of services and applications by individual subscribers with different applications and quality of service requirements.

A possible common platform in the base station of a cellular system will, for example, be able to perform the following function: recognize and communicate with user radio terminals that employ different air interface standards such as GSM, IS-54 and future UMTS. 


\subsubsection{Historical background}

The application of software radio techniques was conceived in the 1970s for military communications [4]-[13]. The first few generations of software radios were developed to provide enhanced fidelity and flexibility unavailable from analog receivers. It could meet changing needs quickly and inexpensively with software upgrades. This flexibility to adapt quickly to difficult problems makes the software radio attractive in commercial telecommunications applications, where infrastructure equipment might otherwise quickly become obsolete due to cumulative growth in markets, rapidly evolving air standards, and requirements for new services.

\subsubsection{Concept of the ideal software radio}

A software radio is a radio whose channel modulation waveforms are defined in software [4]. Ideal software radios employ a combination of techniques that include multiband antennas and RF conversion, wideband analog to digital converter (ADC) and digital to analog converter (DAC), and the implementation of IF, baseband and bitstream processing functions in general-purpose programmable processors. The resulting softwaredefined radio, or "software radio", in part extends the evolution of programmable hardware, increasing flexibility via increased programmability. In part, it represents an ideal that simplifies and illuminates tradeoffs in radio architectures that seek to balance standards compatibility, technology insertion and the compelling economics of today's highly competitive marketplaces. 


\subsubsection{Benefits of SWR for multi-standard systems}

Software radio architectures increase the programmability, simplify hardware component trade-offs and provide new ways of managing the complexity of rapidly emerging standards.

Although the changing standards in part improve the system performance, they also impose costs that create consumer market resistance and dislocation, which in turn, require huge marketing expenditures and economic incentives to entice users and to make the new systems viable. Therefore, software radios are needed as a good solution to this problem raised by the changing of standards.

\subsubsection{Architectures}

Partitioning of functions and components is needed for a specific architecture such that functions are assigned to components and interfaces between components correspond to interfaces between functions. Details will be discussed in Chapter 6 .

\subsubsection{Design}

Ideal software radio design starts with the antenna. The designs for beamforming, diversity and sectorization are fundamental to achieving the improved service quality. The design for the baseband processing is essential to support multi-standards using software radio approaches.

\subsection{Overview and introduction of the smart antenna}

Increased user capacity and expanded geographic coverage demands are the two strong economic motivations regarded by service providers as the impetus for the plan to 
evaluate the ability of the smart antenna to increase CIR for existing TDMA cellular systems, such as GSM and IS-54. Increases of user capacity in existing systems, made by the introduction of various value-added features, such as smart antenna system, and software radio, will provide a timely and economical solution.

\subsubsection{Challenges in wireless communication systems}

We have several challenges to providing efficient and reliable wireless communication. Especially, signal distortion is a serious concern. The wireless channel is plagued by time-varying multipath propagation, caused from reflections and diffractions at surrounding obstacles. The multipath propagation creates signal fading. Flat fading occurs when the time delay spread of multipath is negligible. When the time delay spread is in excess of 0.1 of a symbol period, signal dispersion or inter-symbol interference (ISI) appears as frequency-selective fading [5-7]. In addition to fading distortions, there are system and environmental noise accompanied by adjacent and co-channel interference (ACI and $\mathrm{CCI}$ ), that arise from frequency adjacency and frequency reuse respectively, in wireless cellular systems [8].

There have already been many methods developed to combat signal distortion.

The traditional time domain equalizer is useful for short time delay signals. However, when the delay time is large, the complexity of the equalization system increases.

The smart antenna array, on the other hand, is defined as a group of spatially distributed antennas and proposed as a means to achieve the required longer range and higher spectral efficiency in cellular systems [15-18]. The output of the array antenna is obtained from combining properly each element antenna output. By this operation, it is possible to extract the desired signal from all received signals, even if the same frequency band is occupied by all signals. 
The smart antenna array reduces the interference by using the antenna directivity, improves the carrier-to-interference ratio (C/I) in both uplink and downlink, allows more efficient frequency reuse and the addition of channels until the $\mathrm{C} / \mathrm{I}$ reaches the minimum acceptable level, and provides more capacity. As a result, the whole system capacity can be increased and quality can be improved.

There are two types of smart antenna system: one is the fixed beam and the other is adaptive beam, where a separate beam is formed for each of the users sharing the same time slot and frequency. In a fixed beam system, the handoff from one beam to another happens frequently, while in an adaptive beam smart antenna system, each beam tracks its user within the coverage of the cell.

Much research for spatial and temporal signal processing, using an adaptive antenna, has been pursued in recent years[15]-[18].

\subsubsection{Adaptive algorithms}

There are many adaptive schemes used in the smart antenna to obtain the optimal weights. They can be classified into two groups. One is proposed from a viewpoint of extending techniques of a digital filter, deriving optimal antenna weights adaptively in a time domain such as Least Means Square (LMS), Constant Modulus Algorithm (CMA)[23] etc. The other one is based on Direction of Arrival (DOA) estimation, and is proposed from the viewpoint of spectral analysis in a space domain, such as Discrete Fourier Transforms (DFT)[6], Maximum Entropy Method (MEM)[7] and Multiple Signal Classification (MUSIC)[8]. 


\subsubsection{SDMA}

Space division multiple access (SDMA), is a new concept of access scheme, comparable with FDMA, TDMA and CDMA, and can be combined with them for more user capacity [20]. Using SDMA, users in different angular positions can be served on the same channel, provided the required angular separation between them is satisfied.

One approach in SDMA is the switched multibeam system, in which multiple beams are used to provide the entire coverage of each sector of a base station. The beam with the strongest signal power for the desired user is selected to serve the user. We will use this switched multibeam system in the analysis in Chapter Three.

\subsubsection{Operation}

The operation of the smart antenna is illustrated in Figure 2.1. From the first graph, we find one line-of-sight (LOS) path. The corresponding array response shows a single beam toward the mobile terminal. The second graph introduces two multipaths in addition to the LOS path. The array response is now formed toward the three components with different gain and phase shift and coherently combines the two multipath components with the LOS path. In the third graph, two sources of interference are present with three multipaths. The array response shows corresponding nulls to interference and main gain towards the three multipaths and the LOS path. 


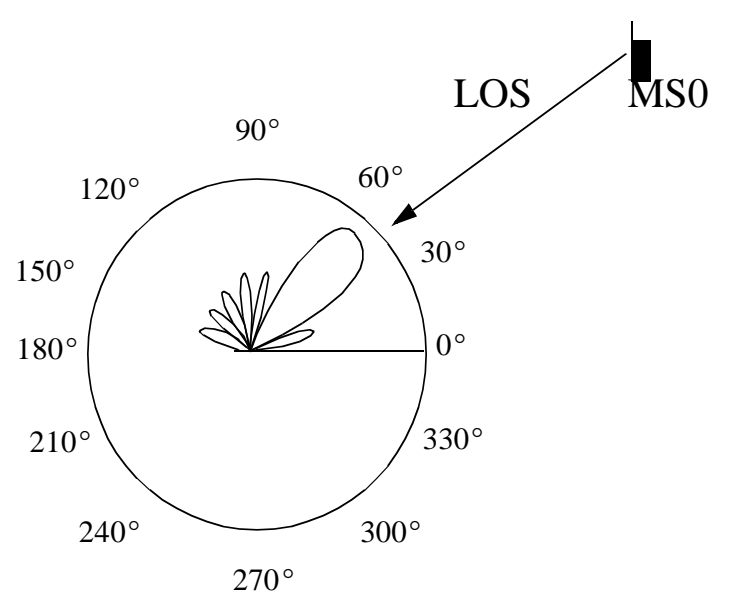

a) array response with no multipath

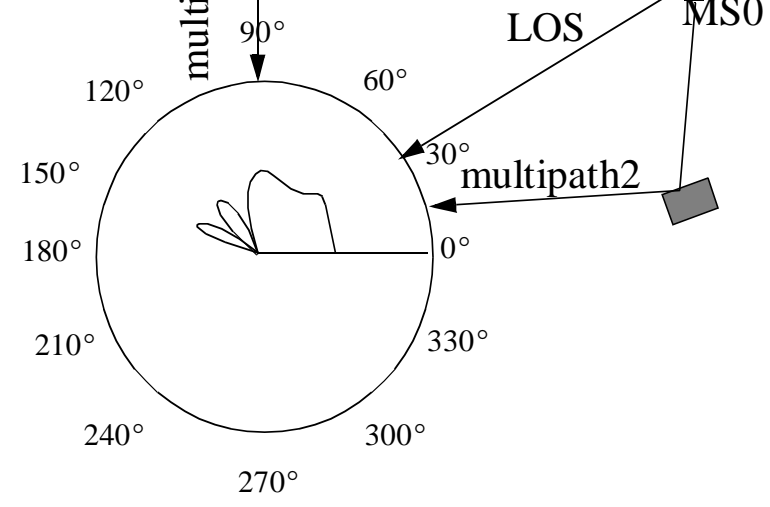

b) array response for multipath

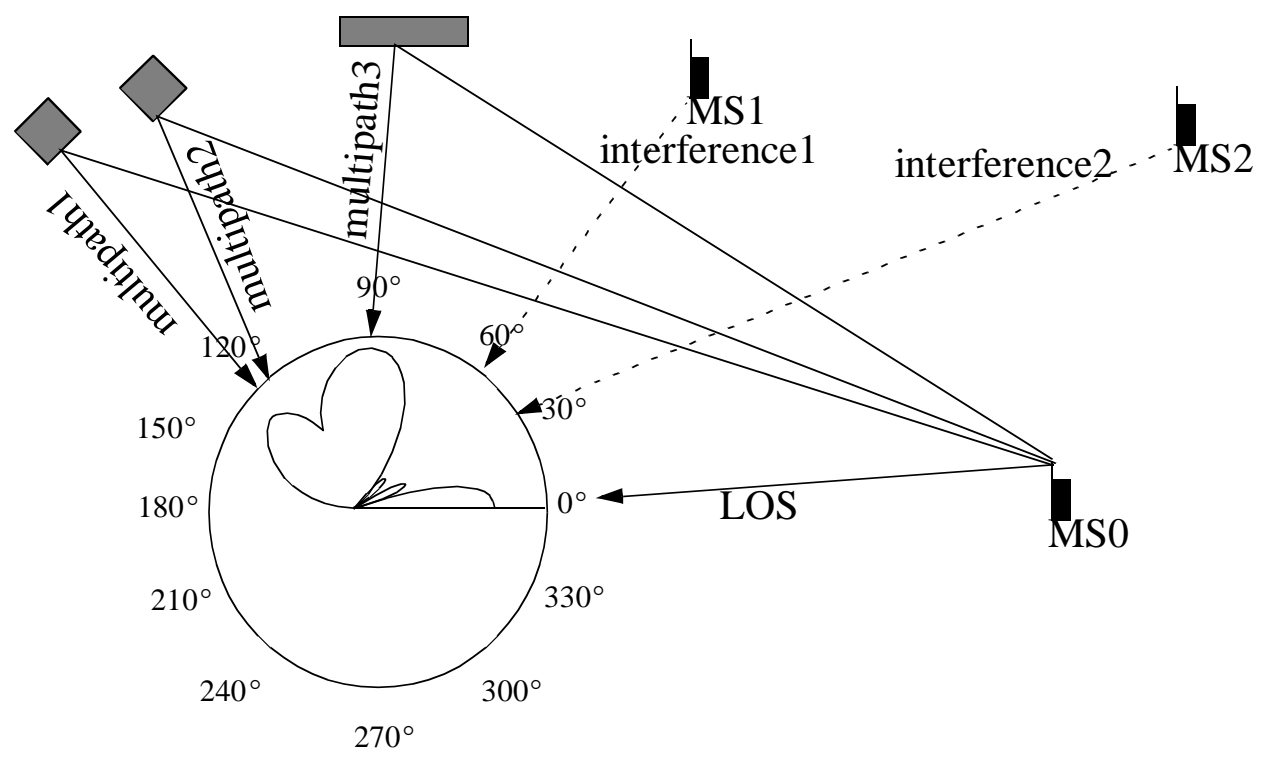

c) array response for combined multipath and interference

Figure 2.1: Array response of the "smart antenna" 


\subsection{Synergies between software radio base stations and smart antennas}

The recognition of architectural synergies between software radio base stations and the adaptive processing needed for smart antennas is increasing. Such synergy can be realized through the solution, where smart antenna is implemented into the base station with a software radio architecture.

The integrated approach enables the processing engine to implement not only air interface demodulation, but also beam steering, advanced signal extraction and interference mitigation concepts, potentially offering substantial system performance enhancements and implementation simplifications.

In this architecture, the sophisticated signal processing algorithms are implemented in DSP software, which can be easily reconfigured to adapt to new wireless environments and new performance requirements. This can be then exploited to achieve more coverage, more capacity, higher quality of service, and higher data rates. The detailed analysis of the down-link performance of smart antennas in the base station is given in Chapter Three.

The software radio architecture provides the flexibility to the implementation with low life-cycle and low cost. And smart antenna, as a typical advanced service to be added into the existing system, serves as a good example to illustrate the concept of software radio. 
Chapter 3

\section{Downlink capacity enhancement using a multibeam smart antenna}

\subsection{Introduction}

There are many techniques that enhance the available capacity (defined as the maximum number of users per $\mathrm{km}^{2}$ that can be served simultaneously), such as cell splitting, hierarchical structure (cell underlayer and overlayer), dynamic channel allocation, and smart antennas. The latter is the most promising due to its large capacity gain and its economical efficiency.

Smart antennas have been one of the hot research topics recently. Their implementation techniques (especially in combination with other techniques, such as power control (PC), DTX and FH), their impact on the capacity for various wireless standards in different environments, and the feasibility of the SDMA as a promising technique for increasing the capacity $\mathrm{N}$-fold( $\mathrm{N}>2)$, have been addressed in [18]-[23].

A smart antenna can be achieved using different implementation techniques, namely switched beam, adaptive beam forming, null forming and optimum combining.

Most of the work reported in the literature is mainly based on software simulation rather than analytical methods, which might need simplifications and/or approximations. However, analytical methods are important and have many advantages due to the short time for getting results compared to simulation methods, and the ability to prove and verify the simulation results. 
In this chapter ${ }^{1}$, we will present (at the system level) a theoretical analysis (down link) for a GSM-like TDMA cellular system and examine the capacity gain with a smart antenna (switched beam).

\subsection{System Model}

\subsubsection{Propagation model}

A wireless channel is normally characterized by path loss, shadowing and fading.

The path loss is an exponential function of the distance between the mobile station (MS) and base station (BS). The path loss exponent $n$ is environment-dependent and is usually taken between 3 to 4 in urban areas. Here, we assume $n$ to be 3.5.

There are two types of fading. Slow fading (namely shadowing) is modeled by the Log-normal distribution with standard deviation $\sigma$ ranges from 6 to $8 \mathrm{~dB}$, varying with environment (in this chapter, we take $6 \mathrm{~dB}$ ). Fast multipath fading, on the other hand, is best modeled by the Raleigh distribution.

The propagation model is given in Equation 3.1 where $P_{t}$ is the transmitted power, $d$ is the distance between the BS and MS, $\lambda$ is a normal random variable corresponding to the shadowing, $F$ is a random variable corresponding to fast multiple fadings, and $g(\theta)$ is the directive gain of the smart antenna (here we assume $g(\theta)=\operatorname{sinc}^{2}(1.65 \theta)$ ).

$$
P_{r}=P_{t} d^{-n} 10^{\frac{\lambda}{10}} F g(\theta)
$$

\footnotetext{
1 The research in this Chapter was done with the assistance of Ph.D student Mohamed Ahmed. He helped derive the related equations (eq 3.11, 3.21), and provided the tool for numerical analysis.
} 
Here we ignore the effect of fast fading, since the techniques such as frequency hopping, equalization and interleaving may greatly reduce it. We assume that the exponential path loss and log-normal shadowing are sufficient to model the wireless channel.

\subsubsection{Frequency planning}

Here we assume a tight 1/3 frequency reuse factor in our study model [3]. This tight frequency plan can only be deployed if $\mathrm{FH}$ is used combined with load control to limit the interference under a certain threshold. The loading factor can be controlled using either a call admission policy or by limiting the number of transceivers at the base station to a smaller value than the available channels.

\subsubsection{Frequency hopping algorithm}

FH is adopted in GSM TDMA systems to improve the performance due to the interference diversity and frequency diversity effect. The co-channel interferers are changed at each time slot, where different sets of interferers with different positions and different shadowing parameters are associated. Therefore an interference diversity is attained. Since the frequency of each mobile station (MS) hops to another value at each time slot, this mitigates the fading and introduces a sort of spectrum spreading.

\subsubsection{Smart antennas}

Smart antennas serve as a spatial filter to reduce interference and can be implemented via various techniques with different levels of complexity and capacity enhancement. Normally, there are four basic structures, namely the switched beam antennas, adaptive beam forming, null forming and optimum combining [18]-[21]. 
- Switched beam antennas

The whole cell may be covered by M beams. The mobile station (MS) is connected to the beam which may provide the best quality in terms of the received power or received signal-interference-ratio (SIR). The MS needs to switch from one beam to another if it crosses the boundary between two beams. The switched beam antenna is considered the simplest due to its non-complex hardware and non-sophisticated weight computation.

- Adaptive beamforming

The arriving direction of the mobile station is estimated and the weights are adjusted to direct the beam to the mobile station. A group of users may be covered by one beam if they are close enough and use different channels for communication. In fact, the algorithms of the direction estimation and weight adjustment are sophisticated and complex and special requirements must be met by the hardware, addressing flexibility and speed.

- Null forming

Interference can be reduced by directing the nulls of the antenna pattern towards the co-channel interferers. Null forming can be implemented in both the uplink and downlink. It is possible to partially cancel M-1 interferers if using an M-element antenna array [19-20]. However, null forming is ineffective for mobile channels due to the multipath nature. If FH is employed, the interferers are changed at each time frame. So it is not practical to use null forming if FH is employed [19-20].

- Optimal combining

In optimal combining, it is not necessary to know the direction of information for a mobile user. Only the desired signal form, or a correlated reference signal, is sufficient for estimation. The weights of the $\mathrm{M}$ elements are adjusted so that all the interferers signals 
which are not correlated with the reference signal will be canceled and as a result the SIR is maximized.

Null forming and optimal combining are not suitable for frequency hopping due to the change of the interferer position and transmitted power in every frame. This requires the positioning of the null towards the interferers, or adjustment of the weight to maximize the CINR must be done within each frame time. Such an implementation is impractical.

In this chapter, we use the switched beam antenna for our study due to its simplicity.

\subsection{Analysis and signal-to-interference ratio calculations for the down-link}

Now we start to calculate the SIR values for TDMA down-links. In our calculation, the switched beams, frequency hopping and DTX are used in the sectorized cell structure.

In order to determine the capacity of the frequency hopping TDMA system which is interference limited, the maximum load (utilization) factor (Erlang/Channel) is found provided that the Cumulative Density Function (CDF) of the CINR (outage probability) is less than the limit, namely a threshold value. In GSM systems, it is required to have the $\mathrm{CDF}(\mathrm{CINR}=9 \mathrm{~dB})=2 \%$. The analysis procedure is shown in Figure 3.1. 


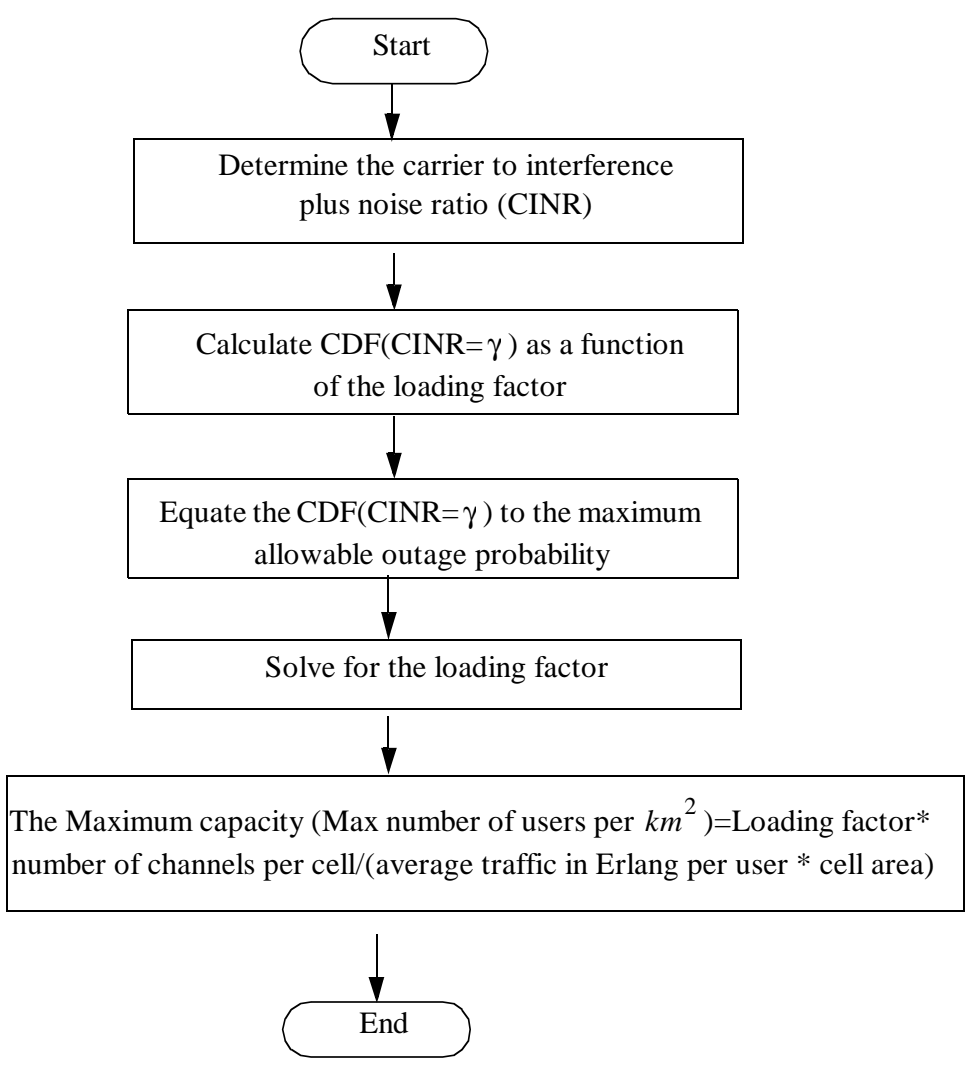

Figure 3.1: Flow chart of analysis procedures 


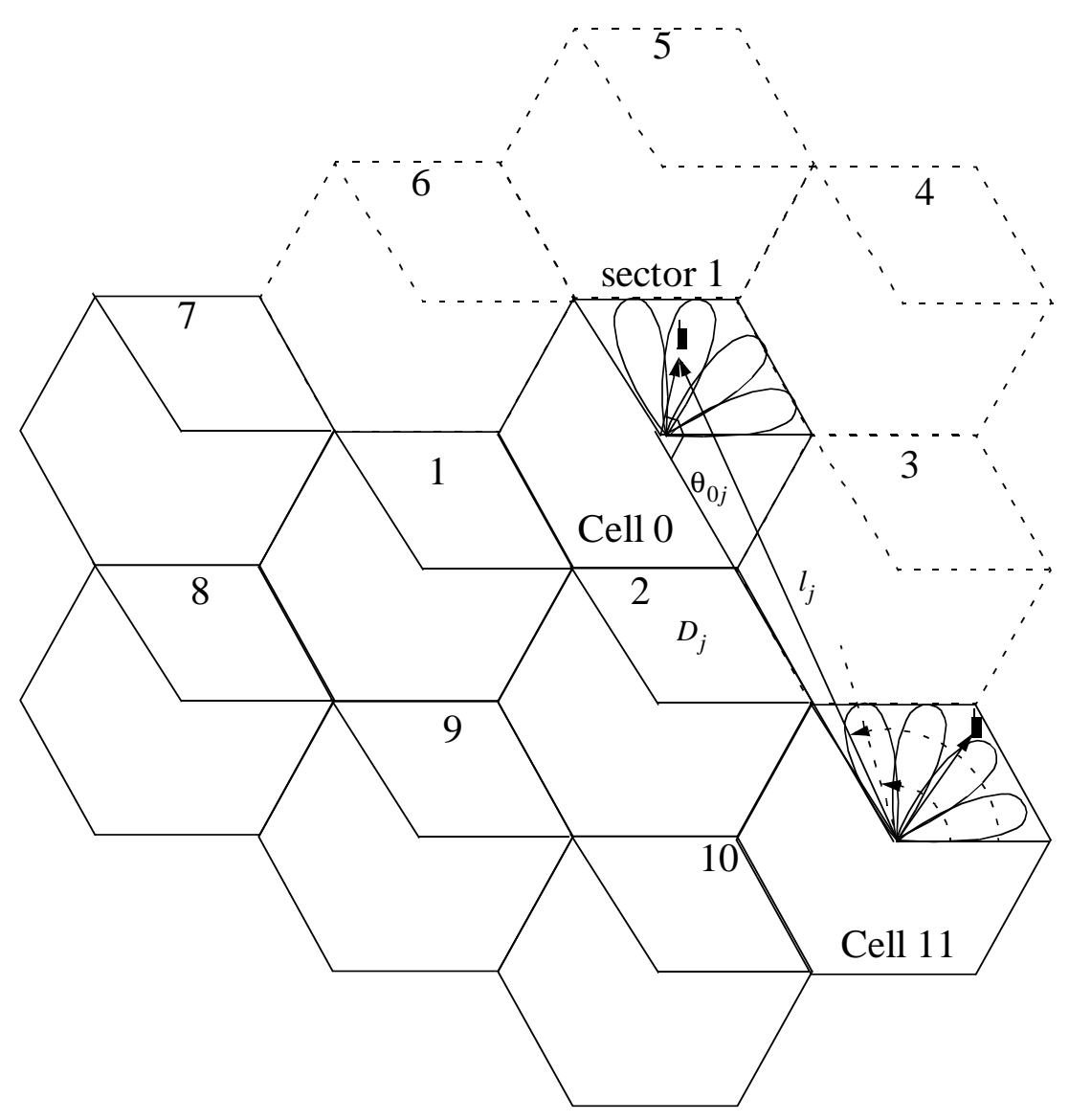

Figure 3.2: Downlink co-channel interference from the first tier interferers and 5 second tier interferers 


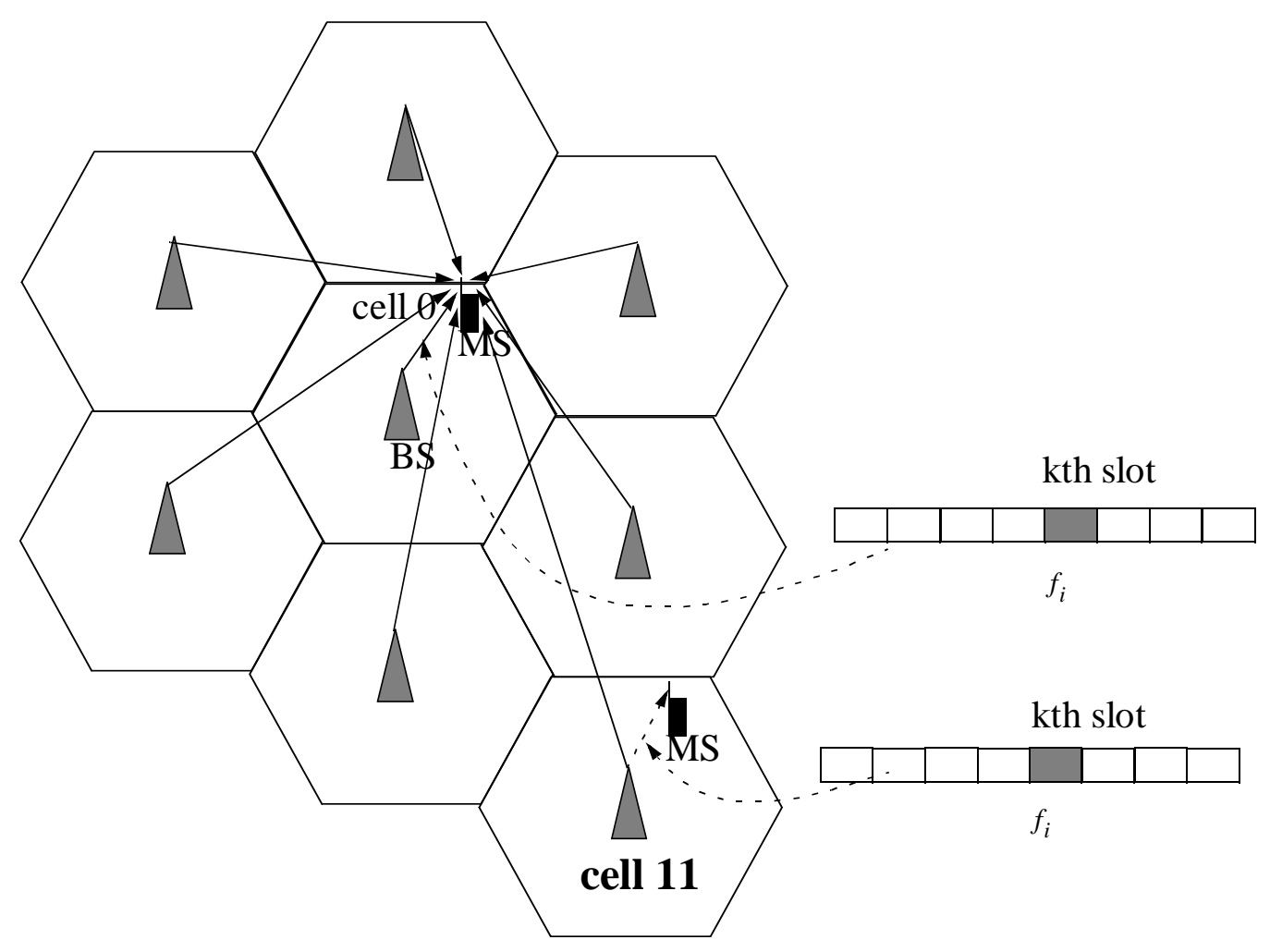

Figure 3.3: Down link co-channel interference with frequency hopping 


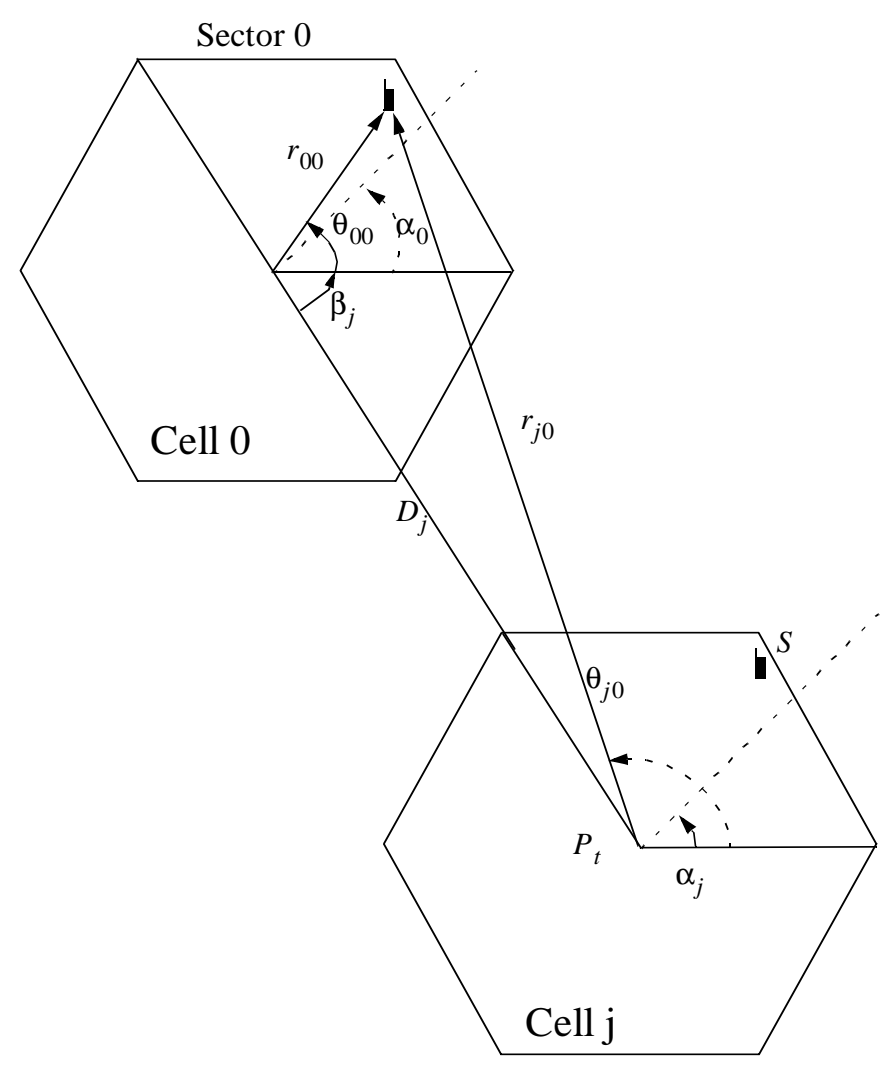

$r_{00}$ : distance from MS to BS in cell 0;

$\theta_{00}:$ angle of MS in cell 0 ;

$\alpha_{0}$ : center angle of the corresponding beam containing MS in cell 0 ;

$\alpha_{j}$ : center angle of the corresponding beam containing MS in cell $\mathrm{j}$;

$D_{j}$ : distance from BS in cell 0 to interference BS in cell $\mathrm{j}$;

$\beta_{j} \quad$ : angle connected from BS in cell 0 to MS in cell 0 and interference BS in cell $\mathrm{j}$;

$\theta_{j 0}$ : angle of connection from BS in cell $\mathrm{j}$ to MS in cell 0 ;

$r_{j 0}:$ distance from MS in cell 0 to interference BS in cell $\mathrm{j}$;

\section{Figure 3.4: Downlink co-channel interference from} interference cell $\mathbf{j}$ without power control 
Figure 3.2 illustrates the downlink interference to a MS in sector 1 in cell 0 from the 11 co-channel interfering cells. The interference from the six first tier BS's (in cells 16) and five second tier BSs (in cells 7-11) is taken into consideration. BS's facing sector 1 (with solid line cell plot) are introducing higher interference than other BS's (with dashed line cell plot), since the former introduce interference from the main lobe while the latter introduce interference from the side lobe.

Figure 3.3 shows the downlink co-channel interference when frequency hopping is used. Consider a mobile that receives in the kth slot on carrier $f_{i}$ in the cell 0. A co-channel interfering BS in the 11th cell creates an interference with this mobile when it communicates to the mobile using the same kth slot of carrier $f_{i}$ in its own cell.

Figure 3.4. and Figure 3.5 illustrates the downlink interference to the mobile station (MS) in cell 0 from one of the interferers cell $\mathrm{j}$ with more details. $P_{j}$ is the transmitted power from the jth interferer BS and is assumed to be a constant $P_{t}$ if no power control is applied (Figure 3.4). It varies as a random variable when perfect power control is assumed (Figure 3.5).

\subsubsection{Switched beam antenna with no power control}

Here we assume the transmitted power from each base station $P_{j}$ is constant, represented by $P_{t}$. The interference power at the MS in cell 0 from the $\mathrm{j}^{\text {th }}$ cell $\left(I_{j}\right)$ is determined from (3.1). Using the problem geometry described in Figure 3.4, thus $I_{j}$ is expressed as:

$$
I_{j}=A_{j} P_{t} r_{j 0}^{-n} 10^{\lambda_{j 0} / 10} g\left(\theta_{j 0}-\alpha_{j}\right)
$$

$j$ is the co-channel interferers index $\left(j=1,2, \ldots, \mathrm{N}_{\text {int }}\right)$; 
$A_{j}$ is a Bernoulli random variable representing the activity of the $\mathrm{j}^{\text {th }}$ interference mobile station. $A_{j}=1$ with probability $\mathrm{q}$ that is equal to the product of the loading factor (LF) with the discontinuous transmission factor (DTXF) [2];

$r_{j 0}$ is the distance between the desired MS and the jth interferer BS;

$\lambda_{j 0}$ is the shadowing parameter of the jth interferer;

$n$ is the path loss exponent;

$g\left(\theta_{j 0}-\alpha_{j}\right)$ is the antenna gain as a function of the interferer angle $\theta_{j 0}$ and beam angle $\alpha_{j}$ defined as $g\left(\theta_{j 0}-\alpha_{j}\right)=\sin c^{2}\left(1.65\left(\theta_{j 0}-\alpha_{j}\right)\right) \cdot[2]$

Because of the interference averaging property of the frequency hopping, we are interested in the mean value of interference power rather than the instantaneous value. The mean value of the interference power from the $\mathrm{j}^{\text {th }}$ cell is expressed as:

$$
\bar{I}_{j}=E\left[I_{j}\right]=E\left(\left[A_{j}\right]\right) P_{t} r_{j 0}^{-n} E\left[10^{\lambda_{j 0} / 10}\right] \sum_{n=1}^{N_{b}} P_{r}\left\{\alpha_{j}=\phi_{n}\right\} g\left(\theta_{j 0}-\phi_{n}\right)
$$

where $P_{r}\left\{\alpha_{j}=\phi_{n}\right\}$ is the probability that the beam angle for the MS in cell $\mathrm{j}$ is equal to $\phi_{n}$, for $\phi_{n} \in \Phi$, where $\Phi$ is the set of possible beam angles. If we assume they are equally distributed, then $P_{r}\left\{\alpha_{j}=\phi_{n}\right\}=\frac{1}{N_{b}}$, where $N_{b}$ is the number of beams. E[] is the mean value of the random variable. Thus it is straightforward to show that $E\left[10^{\lambda_{j} / 10}\right]$ is equal to $\exp \left(\frac{(\alpha \sigma)^{2}}{2}\right)$ where $\alpha=\ln (10) / 10 . \mathrm{E}\left[\mathrm{A}_{\mathrm{j}}\right]$ equals a constant, represented by $q$. 
Rewriting $\bar{I}_{j}$ :

$$
\bar{I}_{j}=q P_{t} r_{j j}^{-n} \exp \left(\frac{(\sigma \alpha)^{2}}{2}\right) \frac{1}{N_{b}} \sum_{n=1}^{N_{b}} g\left(\theta_{j 0}-\phi_{n}\right)
$$

As shown in Figure 3.4, $r_{j 0}$ are functions of $r_{00}, \theta_{00}, D_{j}$ and $\beta_{j}$ :

$$
r_{j 0}=\sqrt{r_{00}^{2}+D_{j}^{2}-2 r_{00} \cdot D_{j} \cdot \cos \left(\beta_{j}+\theta_{00}\right)}
$$

Therefore the total interference $I_{t o t}$ can be expressed as:

$$
I_{t o t}\left(r_{00}, \theta_{00}\right)=\sum_{j=1}^{N_{\text {int }}} \bar{I}_{j}
$$

where $r_{00}$ is the distance between the MS and the desired BS and $\theta_{00}$ is the angle of the MS at the beam with central angle $\alpha_{0}$. The received carrier power at the MS in cell 0 from its $\mathrm{BS}$ is presented by $\mathrm{C}$ and given as:

$$
C=P_{t} r_{00}^{-n} 10^{\frac{\lambda_{00}}{10}} g\left(\theta_{00}-\alpha_{0}\right)
$$

The outage probability $\left(P_{\text {out }}\right)$ is defined as:

$$
P_{\text {out }}\left(r_{00}, \theta_{00}\right)=P(\operatorname{CINR}<\gamma)=\operatorname{CDF}(\operatorname{CINR}=\gamma)=P\left(\left(\frac{C}{N_{0}+I_{\text {tot }}}\right)<\gamma\right)
$$


By substituting for $\mathrm{C}$ from (3.6) the outage probability is expressed as:

$\left.P_{\text {out }}\left(r_{00}, \theta_{00}\right)=P\left(\left(\frac{P_{t} r_{00}^{-n} 10^{\frac{\lambda_{00}}{10}} g\left(\theta_{00}, \alpha_{0}\right)}{N_{0}+I_{\text {tot }}}\right)<\gamma\right)=P\left(10^{\frac{\lambda_{00}}{10}}<\frac{\gamma\left(I_{t o t}\left(r_{00}, \theta_{00}\right)+N_{0}\right)}{P_{t} r_{00}^{-n} g\left(\theta_{00}, \alpha_{0}\right)}\right) 3.8\right)$

$P_{\text {out }}$ is a function of MS position $P_{\text {out }}\left(r_{00}, \theta_{00}\right)$. Using the total probability law, integrated

over the whole area of the sector, the outage probability is given by:

$$
\begin{aligned}
P_{\text {out }} & =\iint_{0}^{\frac{2 \pi}{3} R} P_{0 u t}\left(r_{00}, \theta_{00}\right) f\left(r_{00}, \theta_{00}\right) r_{00} d r_{00} d \theta_{00} \\
P_{\text {out }}= & \int_{0}^{\frac{2 \pi}{3} R} \int_{0}^{R}\left(10^{\frac{\lambda_{00}}{10}}<\frac{\gamma\left(I_{\text {tot }}\left(r_{00}, \theta_{00}\right)\right)}{p_{t} r_{00}^{-n} g\left(\theta_{00}, \alpha_{0}\right)} \mid\left(r_{00}, \theta_{00}\right)\right) f\left(r_{00}, \theta_{00}\right) r_{00} d r_{00} d \theta_{00}
\end{aligned}
$$

where the noise effect is ignored and:

$$
f\left(r_{00}, \theta_{00}\right)=\frac{3}{\pi R^{2}}
$$

Since $\lambda_{00}$ is normally distributed, it is straightforward to show that:

$$
P\left(10^{\frac{\lambda_{00}}{10}}<\frac{\gamma\left(I_{t o t}\left(r_{00}, \theta_{00}\right)\right)}{p_{t} r_{00}^{-n} g\left(\theta_{00}, \alpha_{0}\right)} \mid\left(r_{00}, \theta_{00}\right)\right)=1-Q\left(\frac{\beta}{\sigma}\right)
$$

where:

$$
\beta=10 \log \left(\frac{\gamma\left(I_{t o t}\left(r_{00}, \theta_{00}\right)\right)}{p_{t} r_{00}^{-n} g\left(\theta_{00}, \alpha_{0}\right)}\right)
$$


Therefore the outage probability is given by:

$$
P_{\text {out }}=\int_{0}^{\frac{2 \pi}{3}} \int_{0}^{R} P\left(\left(\frac{C}{I_{\text {tot }}}\right)<\gamma \mid\left(r_{00}, \theta_{00}\right)\right) f\left(r_{00}, \theta_{00}\right) r_{00} d r_{00} d \theta_{00}=\int_{0}^{\frac{2 \pi}{3}} \int_{0}^{R}\left(1-Q\left(\frac{\beta}{\sigma}\right)\right) f\left(r_{00}, \theta_{00}\right) r_{00} d r_{00} d \theta_{00}
$$

The outage probability is determined from (3.10) by solving the double integration numerically at different loading values to find the maximum loading factor and then the system capacity as presented in the next section.

\subsubsection{Multibeams antenna with perfect power control}

With perfect power control, each mobile in the $\mathrm{j}^{\text {th }}$ interfering cell receives a constant power (S) from its BS irrespective of its location. To keep the received power at each MS constant, each BS adjusts its transmitted power at BS according to each MS's location within its cell. The transmitted power is a function of $r_{j j}$ and $\theta_{j j}$ (shown in Figure 3.4), expressed as:

$$
P_{t}\left(r_{j j}, \theta_{j j}\right)=S r_{j j}^{n} 10{ }^{-\frac{\lambda_{j i}}{10}}\left[g\left(\theta_{j j}-\alpha_{j}\right)\right]^{-1}
$$

where $\lambda_{j j}$ is the shadowing factor of the downlink path. From (3.2), the downlink interference from cell $\mathrm{j}$ is: 


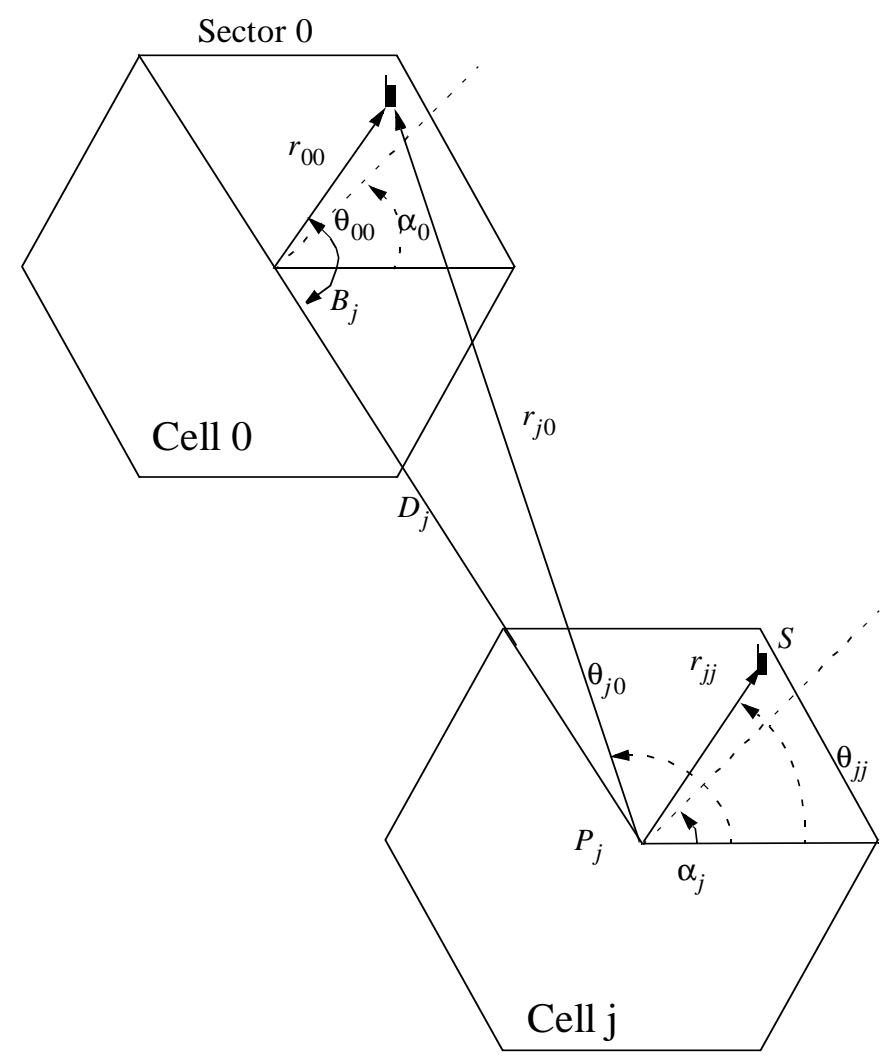

$r_{00}:$ distance from MS to BS in cell 0 ;

$\theta_{00}:$ angle of MS in cell 0 ;

$\alpha_{0}$ : center angle of the corresponding beam containing MS in cell 0 ;

$r_{j j}:$ distance from interference MS to BS in cell $\mathrm{j}$;

$\theta_{j j} \quad$ : angle of MS in interference cell $\mathrm{j}$;

$\alpha_{j}$ : center angle of the corresponding beam containing MS in cell $\mathrm{j}$;

$D_{j}$ : distance from BS in cell 0 to interference BS in cell $\mathrm{j}$;

$B_{j} \quad$ : angle connected from BS in cell 0 to MS in cell 0 and interference BS in cell $\mathrm{j}$;

$\theta_{j 0}:$ angle of connection from BS in cell $\mathrm{j}$ to MS in cell 0 ;

$r_{j 0}:$ distance from MS in cell 0 to interference BS in cell $\mathrm{j}$;

Figure 3.5: Downlink co-channel interference from interference cell $\mathbf{j}$ with perfect power control 


$$
I_{j}\left(r_{j j}, \theta_{j j}\right)=A_{j} S\left(\frac{r_{j j}}{r_{j 0}}\right)^{n} 10 \frac{\lambda_{j 0}-\lambda_{i j}}{10} \frac{g\left(\theta_{j 0}, \alpha_{j}\right)}{g\left(\theta_{j j}, \alpha_{j}\right)}
$$

and the mean value of the interference is the integration in sector area $a$ :

$$
\begin{aligned}
& \bar{I}_{j}\left(r_{00}, \theta_{00}\right)=E\left[\iint_{a} I_{j}\left(r_{j j}, \theta_{j j}\right) f(a) d a\right] \\
& \bar{I}_{j}\left(r_{00}, \theta_{00}\right)=S E\left[A_{j}\right] E\left[10^{\frac{\lambda_{j}-\lambda_{j j}}{10}}\right] \iint_{a} \frac{r_{j j}^{n}}{r_{j 0}^{n}} \frac{g\left(\theta_{j 0}, \alpha_{j}\right)}{g\left(\theta_{j j}, \alpha_{j}\right)} f(a) d a
\end{aligned}
$$

i.e.:

$$
\bar{I}_{j}\left(r_{00}, \theta_{00}\right)=\frac{3 S r_{j 0}^{-n}}{\pi R^{2}} E\left[A_{j}\right] E\left[10^{\frac{\lambda_{j}-\lambda_{j j}}{10}}\right] \int_{0}^{\frac{2 \pi}{3}} \int_{0}^{R} r_{j j}^{n} \frac{g\left(\theta_{j 0}, \alpha_{j}\right)}{g\left(\theta_{j j}, \alpha_{j}\right)} r_{j j} d r_{j j} d \theta_{j j}
$$

which can be simplified as:

$$
\bar{I}_{j}\left(r_{00}, \theta_{00}\right)=\frac{3 S q r_{j 0}^{-n}}{\pi R^{2}} E\left[10^{\frac{\lambda_{j}-\lambda_{j j}}{10}}\right] h_{j}
$$

where

$$
\begin{gathered}
h_{j}=\int_{0}^{\frac{2 \pi}{3}} \int_{0}^{R} r_{j j}^{n} \frac{g\left(\theta_{j 0}, \alpha_{j}\right)}{g\left(\theta_{j j}, \alpha_{j}\right)} r_{j j} d r_{j j} d \theta_{j j} \\
E\left[10^{\frac{\lambda_{j}-\lambda_{i j}}{10}}\right]=\exp \left((\alpha \sigma)^{2}\right)
\end{gathered}
$$


$h_{j}$ is written as:

$$
h_{j}=\left.\frac{r_{j j}^{n+2}}{n+2}\right|_{0} ^{R} \int_{0}^{\frac{2 \pi}{3}} \frac{g\left(\theta_{j 0}, \alpha_{j}\right)}{g\left(\theta_{j j}, \alpha_{j}\right)} d \theta_{j j}=\frac{R^{n+2}}{n+2} \int_{0}^{\frac{2 \pi}{3}} \frac{\sin c^{2} 1.65\left(\alpha_{j}-\theta_{j 0}\right)}{\sin c^{2} 1.65\left(\alpha_{j}-\theta_{j j}\right)} d \theta_{j j}
$$

simplifying (3.16):

$$
h_{j}=\frac{R^{n+2}}{n+2} Y_{j}\left(\theta_{00}, r_{00}\right)
$$

where

$$
Y_{j}\left(\theta_{00}, r_{00}\right)=\int_{0}^{\frac{2 \pi}{3}} \frac{\sin c^{2} \alpha\left(\alpha_{j}-\theta_{j 0}\right)}{\sin c^{2} \alpha\left(\alpha_{j}-\theta_{j j}\right)} d \theta_{j j}
$$

So the total interference $I_{\text {tot }}$ from the $N_{\text {int }}$ interferers is evaluated by:

$$
I_{t o t}\left(r_{00}, \theta_{00}\right)=\sum_{j=1}^{N_{i n t}} \bar{I}_{j}\left(r_{00}, \theta_{00}\right)
$$

Substituting by (3.15) and (3.16) to (3.17):

$$
I_{t o t}\left(r_{00}, \theta_{00}\right)=\frac{R_{j}^{n}}{n+2} \frac{3 S}{\pi} q \exp \left((\alpha \sigma)^{2}\right) \sum_{j=1}^{N_{i n t}} r_{j 0}^{-n}\left(\theta_{00}, r_{00}\right) Y_{j}\left(\theta_{00}, r_{00}\right)
$$

Since perfect downlink power control is assumed, the received carrier power at the MS from the desired BS is a constant: $C=S$ and as a result the outage probability is given by: 


$$
P_{\text {out }}=P\left(\left(\frac{S}{I_{\text {tot }}}\right)<\gamma\right)=P\left(I_{\text {tot }}\left(r_{00}, \theta_{00}\right)>\left(\frac{S}{\gamma}\right)\right)
$$

Using the total probability law, the final outage probability is given by:

$$
\begin{aligned}
& \frac{2 \pi}{3} R \\
& P_{\text {out }}=\int_{0} \int_{0} P\left(I_{\text {tot }}\left(r_{00}, \theta_{00}\right)>\frac{S}{\gamma} \mid\left(r_{00}, \theta_{00}\right)\right) f\left(r_{00}, \theta_{00}\right) r_{00} d r_{00} d \theta_{00} \\
& \frac{2 \pi}{3} R \\
& P_{\text {out }}=\int_{0} \int_{0} u\left(I_{\text {tot }}\left(r_{00}, \theta_{00}\right)-\frac{S}{\gamma}\right) f\left(r_{00}, \theta_{00}\right) r_{00} d r_{00} d \theta_{00}
\end{aligned}
$$

where $\mathrm{u}(\mathrm{x})$ is the unit step function defined as $\left\{\begin{array}{l}1(X>0) \\ 0(X<0)\end{array}\right.$

By computing the above integrals and summation numerically, we can solve for the maximum loading factor that satisfies the outage probability condition. By determining the maximum loading factor $\left(L F_{\text {max }}\right)$, the system capacity, defined as the number of users per unit area, can be evaluated by:

$$
\text { Capacity }=\frac{L F_{\text {max }} C H_{\text {cell }}}{E_{\text {user }} A_{\text {cell }}}
$$

where $\mathrm{CH}_{\text {cell }}$ is the number of channels per cell, $E_{\text {user }}$ is the average traffic in Erlangs per user, and $A_{\text {cell }}$ is the cell area. 


\subsubsection{Results}

Now let us take a numerical example to illustrate the methodology of determining the system capacity using the analysis presented above.

The parameters listed below are used in the numerical analysis and they are chosen to be as close as possible to the actual parameters of the GSM wireless networks, to have realistic results:

- Cell radius $=2 \mathrm{~km}$

- Number of sectors $=3$

- Number of beams per sector $\left(\mathrm{N}_{\mathrm{b}}\right)=4$

- Number of co-channel interferers $=11$ ( 6 from the first tier +5 from the second tier)

- Discontinuous Transmission Factor $(\mathrm{DTXF})=0.5$

- $\mathrm{Ch}_{\text {cell }}=54(7$ Carriers: $1 \mathrm{BCCH}+6 \mathrm{FH}$ carriers $)$

- $\mathrm{Erl}_{\mathrm{user}}=0.02 \mathrm{Erl} / \mathrm{user}$ 
Table 3.1: Outage Probability $(\operatorname{CDF}(\mathrm{CIR}=9 \mathrm{~dB}))$ at different loading factor values

\begin{tabular}{|l|l|r|r|r|r|r|l|}
\hline \multirow{2}{*}{$\begin{array}{l}\text { Antenna } \\
\text { Type }\end{array}$} & \multicolumn{5}{|c|}{ CDF $(\mathrm{CIR}=9 \mathrm{~dB})$} \\
\cline { 3 - 8 } & $\mathrm{LF}=10 \%$ & $\mathrm{LF}=20 \%$ & $\mathrm{LF}=30 \%$ & $\mathrm{LF}=40 \%$ & $\mathrm{LF}=50 \%$ & $\mathrm{LF}=60 \%$ \\
\hline \hline \multirow{2}{*}{ no PC } & 3 sectors & $2.9 \times 10^{-02}$ & $5.7 \times 10^{-02}$ & $8.1 \times 10^{-02}$ & $1.0 \times 10^{-01}$ & $1.2 \times 10^{-01}$ & $1.3 \times 10^{-01}$ \\
\cline { 2 - 8 } & Multiple Beam & $7.5 \times 10^{-03}$ & $2.0 \times 10^{-02}$ & $3.3 \times 10^{-02}$ & $4.5 \times 10^{-02}$ & $5.6 \times 10^{-02}$ & $6.7 \times 10^{-02}$ \\
\hline \multirow{3}{*}{ with PC } & 3 sectors & $1 \times 10^{-05}$ & $6.0 \times 10^{-02}$ & $1.3 \times 10^{-01}$ & $2.4 \times 10^{-01}$ & $4.1 \times 10^{-01}$ & $6.3 \times 10^{-01}$ \\
\cline { 2 - 8 } & Multiple Beam & $1 \times 10^{-05}$ & $1 \times 10^{-05}$ & $1 \times 10^{-05}$ & $1 \times 10^{-05}$ & $1 \times 10^{-05}$ & $2.0 \times 10^{-02}$ \\
\hline
\end{tabular}

Table 3.2: Maximum loading factor (percentage) with various outage probability requirements

\begin{tabular}{|l|c|c|c|c|c|c|}
\hline \multirow{3}{*}{ Antenna type } & \multicolumn{5}{|c|}{ Maximum Loading Factor $\left(\mathrm{LF}_{\max }\right)$} \\
\cline { 2 - 7 } & \multicolumn{2}{|c|}{$\mathrm{p}_{\text {out }}=2 \%$} & \multicolumn{2}{|c|}{$\mathrm{p}_{\text {out }}=4 \%$} & \multicolumn{2}{c|}{$\mathrm{p}_{\text {out }}=10 \%$} \\
\cline { 2 - 7 } & with PC & No PC & with PC & No PC & with PC & No PC \\
\hline \hline 3 sectors & $18 \%$ & $7 \%$ & $19 \%$ & $15 \%$ & $26 \%$ & $40 \%$ \\
\hline Multiple Beam & $60 \%$ & $20 \%$ & $70 \%$ & $36 \%$ & $100 \%$ & $90 \%$ \\
\hline
\end{tabular}

The outage probability is evaluated by simulation and analytically using (3.11) and (3.21) at different loading values.

Figure 3.6 to Figure 3.8 show the $\mathrm{CDF}(\mathrm{CIR})$ with Multiple Beams (MB) or sectorization(SC) only without Power Control (PC) at 20\%, 40\% and 60\% loading factor respectively. From these figures, we may see the great performance improvement using multiple beams instead of sectorization only.

Figure 3.9 and Figure 3.10 show the $\mathrm{CDF}(\mathrm{CIR})$ at various loading factors with multiple beams or sectorization base station respectively. As the cell loading rises, the CDF increases at certain CIR.

Figure 3.11, shows that with a certain outage probability, cell loading is higher with switched beam than the sectorization only. 
Figure 3.12 to Figure 3.17 show the $\mathrm{CDF}(\mathrm{CIR})$ with multiple beams or sectorization only with and without Power Control (PC) at 20\%, 40\% and 60\% loading factors respectively. Both linear and logarithmic presentation format are used. From those figures, we may see that system performance can be greatly enhanced by using multiple beam combined with power control.

Using Figure 3.18 and Figure 3.19, the outage probability $(\mathrm{P}(\mathrm{CIR}<9 \mathrm{~dB})=\mathrm{CDF}(\mathrm{CIR}=9 \mathrm{~dB}))$ is determined for these different cases. The outage probability is plotted versus the loading factor in Figure 3.20. The two figures show that significant enhancement in the performance can be achieved by employing multiple beam antennas and power control. It can be shown that the outage probability can be reduced by $40-65 \%$ without PC, while the reduction is more than $95 \%$ with PC, particularly at low and medium loading factors.

Table 3.1 lists the outage probability for the different cases mentioned above and at loading values of $10-60 \%$. It is apparent that the outage probability is highly reduced by using multiple beam antennas, especially when it is combined with power control, e.g. with $\mathrm{MB}, \mathrm{P}_{\text {out }}$ is reduced from $1.2 \times 10^{-1}$ to $6.7 \times 10^{-2}$ without PC, and to $2.0 \times 10^{-2}$ with PC.

Table 3.2 lists the maximum loading factor at different maximum outage probability requirements $\left(\mathrm{p}_{\text {out }}\right)$. For example, when $\mathrm{p}_{\text {out }}=2 \%$ the maximum loading factor (with no PC) is increased from $7 \%$ to $20 \%$ (which is almost threefold) by using MB without PC and by employing PC without MB it can be increased to $18 \%$, which is more than 2.5 times the original loading factor. By combining both techniques, the maximum loading factor jumps to $60 \%$, which is more than an eighthfold gain. This high gain is achieved due to the multiplicative effect of both techniques MB and PC. However, the capacity gain might be less in reality because of the difficulty of having such perfect power control.The gain due to PC is inversely proportional to the loading factor and it is shown that it can degrade the performance, especially without MB, e.g. the maximum loading is reduced. 
Figure 3.6: CDF of CIR for a cell at 20\% Load Factor 
Figure 3.7: CDF of CIR for a cell at 40\% Load Factor 
Figure 3.8: CDF of CIR for a cell at $60 \%$ Load Factor 
Figure 3.9: CDF of CIR for 3-sector antenna without switched beam (no power control) 
Figure 3.10: CDF of CIR for 3-sector antenna with switched beam (no power control) 
Figure 3.11: CDF of load factor with CIR = 9dB 
Figure 3.12: CDF of CIR for a cell at $20 \% \mathrm{Load}$ Factor 
Figure 3.13: CDF of CIR for a cell at 20\% Load Factor 
Figure 3.14: CDF of CIR for a cell at $40 \%$ Load Factor 
Figure 3.15: CDF of CIR for a cell at $40 \%$ Load Factor 
Figure 3.17: CDF of CIR for a cell at $60 \% \mathrm{Load}$ Factor 
Figure 3.18: CDF of CIR for 3 sector antenna without switched beam under power control 
Figure 3.19: CDF of CIR for 3 sector antenna with switched beam under power control 
Figure 3.20: Outage probability as function of load factor with $\mathrm{CIF}=9 \mathrm{~dB}$ 
Chapter 4

\title{
SWR Architecture for Base Station in TDMA system
}

\begin{abstract}
In both GSM and IS-54 TDMA systems, a lot of functions like channel coding, modulation and equalization, are used in a similar way. This makes possible a parameterized software implementation. It enables the air interface to be changed easily by only exchanging a set of parameters for the reconfiguration, rather than downloading the whole software of a system.
\end{abstract}

This chapter describes the parameterized software implementation of the baseband processing functions in GSM and IS-54 systems. The parameterized method is applied to several main functions of the two systems: channel coding, interleaving and modulation. The baseband architecture for the common modulator and the common equalizer is proposed.

\subsection{Baseband processing function review for GSM and IS-54}

\subsubsection{Review of the GSM system}

The GSM system is widely implemented in Europe, and also in U.S. and Canadian PCS systems, in the $1800 \mathrm{MHz}$ band. GSM is more efficient in spectrum utilization than AMPS analog cellular, using $25 \mathrm{kHz}$ per user versus $30 \mathrm{kHz}$ per user for the latter. 
Each of the frequency channels is segmented into 8 slots of length $0.577 \mathrm{~ms}$ (120/ $26 * 8 \mathrm{~ms})$. The 8 time slots makes up a TDMA frame of length $4.615 \mathrm{~ms}$ (120/26 ms). The recurrence of one particular time slot every 4.615 ms makes up one basic channel.

Here in the GSM system, we consider only the Traffic Channel/Full-Rate Speech (TCH/FR) used to carry speech at $13 \mathrm{kbps}$.

Figure 4.1 gives the block diagram of a transmitter and receiver (channel decoding) for a GSM base station.

The physical structure of hyperframe, superframe, multiframe, frame, and time slot for a GSM system is given in Figure 4.2.

A more detailed time slot structure for the GSM system is given in Figure 4.3.

TCHs for the uplink and downlink are separated in time by 3 burst periods, so that the mobile does not have to transmit and receive simultaneously. TCHs are defined using a 26-frame multiframe (i.e a group of 26 TDMA frames). The length of a 26-frame multiframe is $120 \mathrm{~ms}$, which is how the length of a burst period is defined $(120 \mathrm{~ms} / 26$ frames/8 burst periods per frame). Out of the 26 frames, 24 are used for traffic, one is used for the Slow Associated Control Channel (SACCH) and one is currently unused. 

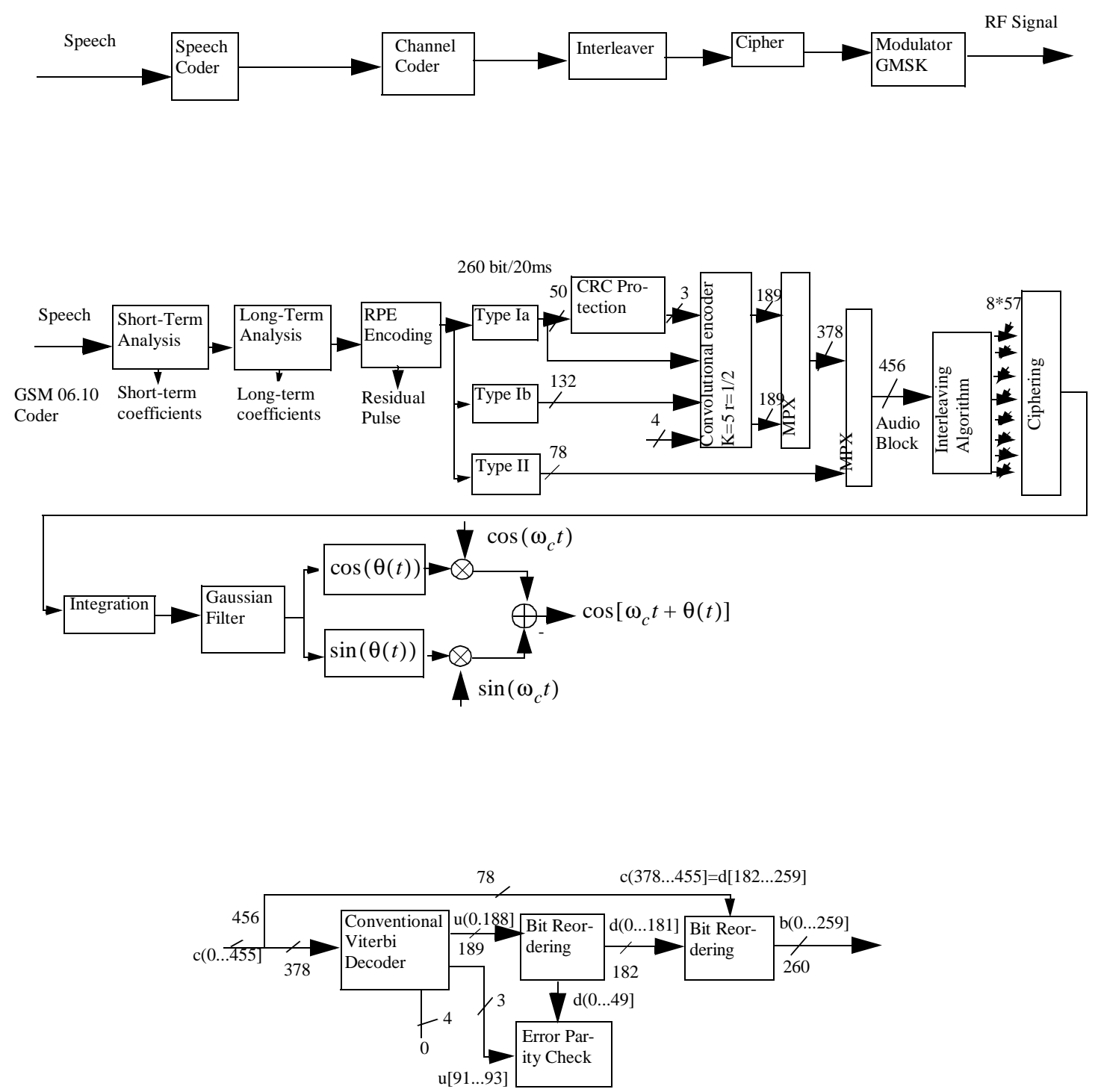

Figure 4.1: A block diagram of transmitter and receiver (channel decoding) for a GSM base station 


\section{- The GSM speech coding}

The full rate speech codec in GSM is described as Regular Pulse Excitation with Long Term Prediction (GSM 06.10 RPE-LTP). Basically, the encoder divides the speech into short-term predictable parts, long-term predictable parts and the remaining residual pulse. Then, it encodes that pulse and parameters for the two predictors. The decoder reconstructs the speech by passing the residual pulse first through the long-term prediction filter, and then through the short-term predictor.

\section{- The GSM channel coding}

Channel coding introduces redundancy into the data flow in order to allow the detection or even the correction of bit errors introduced during the transmission.

The speech coding algorithm produces a speech block of 260 bits every 20 ms (i.e. bit rate $13 \mathrm{kbit} / \mathrm{s})$. In the decoder, these speech blocks are decoded and converted to 13 bit uniformly coded speech samples. The 260 bits of the speech block are classified into two groups. The 78 Class II bits are considered of less importance and are unprotected. The 182 Class I bits are split into 50 Class Ia bits and 132 Class Ib bits. Class Ia bits are first protected by 3 parity bits for error detection. Class Ib bits are then added together with 4 tail bits before applying the convolutional code with rate $\mathrm{r}=1 / 2$ and constraint length $\mathrm{K}=5$. The resulting 378 bits are then added to the 78 unprotected Class II bits resulting in a complete coded speech frame of 456 bits. 


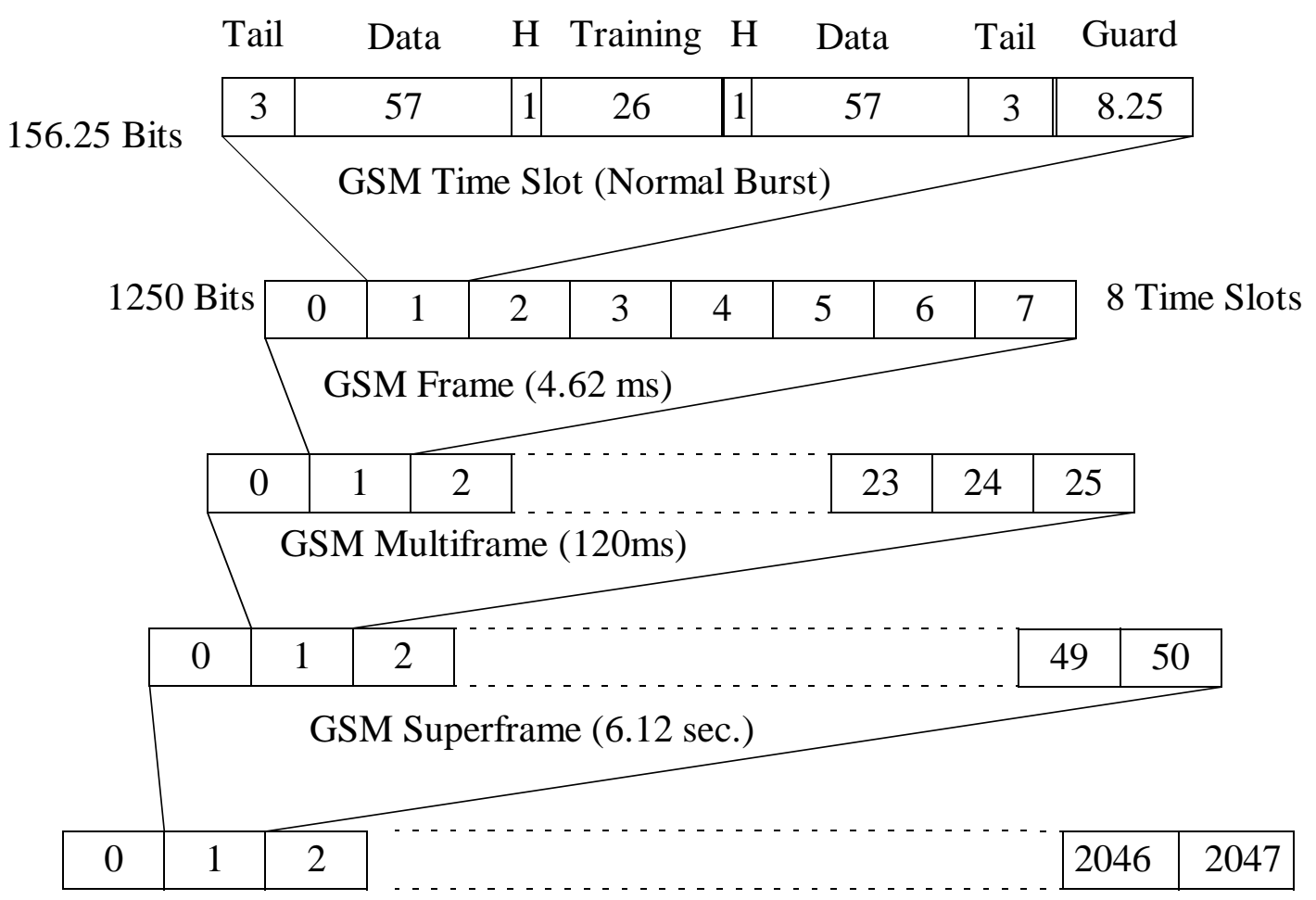

GSM Hyperframe (3.48h)

Figure 4.2: Physical structure of hyperframe, superframe, multiframe, frame, and time slot for a GSM system 
- Error detecting codes

The GSM standard uses a 3-bit error redundancy code to enable assessment of the correctness of the bits which are more sensitive to errors in the speech frame (the category Ia 50-bits). The polynomial representing the detection code for category Ia bits is $G(X)=X^{3}+X+1$.

At the receiving side, the same operation is done and if the remainder differs, an error is detected and the audio frame is eventually discarded.

- Convolutional coding/decoding

Convolutional coding consists in transmitting the result of convolutions of the source sequence according to different convolution formulas. The GSM convolutional code involves in adding 4 bits (set to " 0 ") to the initial 185 bit sequence and then applying two different convolutions: polynomials are respectively $\mathrm{G} 1(\mathrm{X})=\mathrm{X}^{4}+\mathrm{X}^{3}+1$ and $\mathrm{G} 2(\mathrm{X})=$ $\mathrm{X}^{4}+\mathrm{X}^{3}+\mathrm{X}+1$. The final result is composed of two 189 bit sequences.

Convolutional decoding can be performed using a Viterbi algorithm [46]. One important parameter for this algorithm is the constraint length $\mathrm{K}$, which is the encoder memory length. A Viterbi decoder in steady-state operation then keeps $2^{K-1}$ paths. The details are given in [46]. 


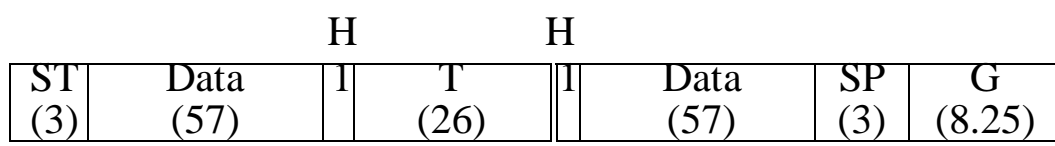

Normal Burst

\begin{tabular}{|c|c|c|c|}
\hline ST & Fixed Bits & SP & G \\
$(3)$ & $(142)$ & $(3)$ & $(8.25)$ \\
\hline
\end{tabular}

Frequency Correction Burst

\begin{tabular}{|c|c|c|c|c|c|}
\hline ST & Data & T & Data & SP & G \\
$(3)$ & $(39)$ & $(64)$ & $(39)$ & $(3)$ & $(8.25)$ \\
\hline
\end{tabular}

Synchronization Burst

\begin{tabular}{|c|c|c|c|c|c|}
\hline ST & Data & T & Data & SP & G \\
$(3)$ & $(58)$ & $(26)$ & $(58)$ & $(3)$ & $(8.25)$ \\
\hline
\end{tabular}

Dummy Burst

\begin{tabular}{|l|c|c|c|c|}
\hline ST & Synch Seq & Data & SP & G \\
$(3)$ & $(41)$ & $(36)$ & $(3)$ & $(68.25)$ \\
\hline
\end{tabular}

$\underline{\text { Access Burst }}$

ST: Start Bits

SP: Stop Bits

$\mathrm{H}$ : Stealing Bits

$\mathrm{T}$ : Training Bits

G: Guard Time

Figure 4.3: GSM time slot structure 
The GSM convolutional coding rate per data flow is 378 bits each $20 \mathrm{~ms}$, i.e.: 18.9 $\mathrm{kb} / \mathrm{s}$. However, before this signal is modulated, the 78 unprotected Class II bits are added. Thus, the GSM bit rate per flow is 456 bits each $20 \mathrm{~ms}$ i.e. $22.8 \mathrm{~kb} / \mathrm{s}$.

\section{- Interleaving/de-interleaving}

Interleaving is meant to de-correlate the relative positions of the bits respectively in the code words and in the modulated radio bursts. The aim of the interleaving algorithm is to avoid the risk of losing consecutive data bits. GSM blocks of full rate speech are interleaved on 8 bursts: the 456 bits of one block are split into 8 bursts in sub-blocks of 57 bits each. A sub-block is defined as either the odd- or the even-numbered bits of the coded data within one burst. Each sub-block of 57 bits is carried by a different burst and in a different TDMA frame. Hence, a burst contains the contribution of two successive speech blocks, A and B. In order to destroy the proximity relations between successive bits, bits of block A use the even position inside the burst and bits of block B, the odd position.

De-interleaving consists in performing the reverse operation. The major drawback of interleaving is the corresponding delay: transmission time from the first burst to the last one in a block is equal to 8 TDMA frames (i.e. about $37 \mathrm{~ms}$ ).

\section{- Ciphering/deciphering}

A protection has been introduced in GSM by means of transmission ciphering. The ciphering method does not depend on the type of data to be transmitted (speech, user data or signaling) but is only applied to normal bursts.

Ciphering is achieved by performing an "exclusive or" operation between a pseudo-random bit sequence and the 114 useful bits of a normal burst (i.e. all information bits except the 2 stealing flags). The pseudo-random sequence is derived from the burst number and a key session established previously through signaling means. Deciphering follows exactly the same operation. 


\subsubsection{Review of IS-54 system}

IS-54, also known as the D-AMPS standard, provides three TDMA voice channels in the space of one $30 \mathrm{kHz}$ analog channel. IS-54 is used as the analysis example. Figure 4.4 gives the coding and interleaving functional block diagram in IS-54.

The time slot structure for IS-54 is given in Figure 4.5.

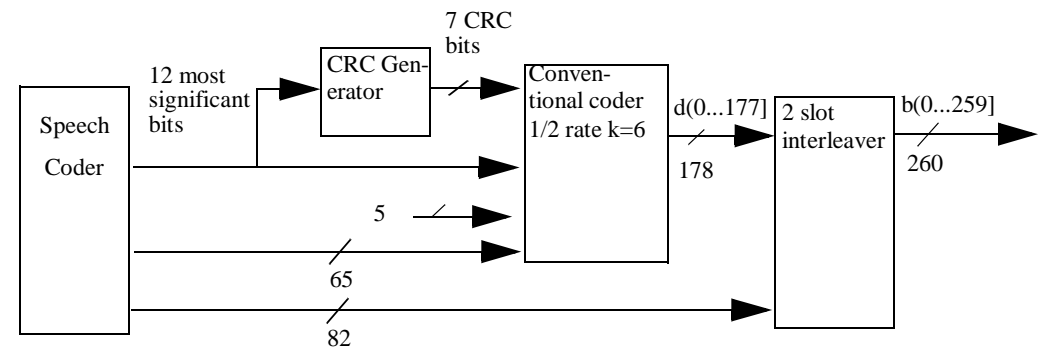

Figure 4.4: Coding and interleaving in IS-54 
$6.67 \mathrm{msec}$

\begin{tabular}{|c|c|c|c|c|c|c|c|}
\hline G & R & Data & Sync & Data & SACCH & CDVCC & Data \\
$(6)$ & $(6)$ & $(16)$ & $(28)$ & $(122)$ & $(12)$ & $(12)$ & $(122)$ \\
\hline
\end{tabular}

$\underline{\text { Reverse Link Burst (Mobile station to base station) }}$

\begin{tabular}{|c||c|c|c|c|c|}
\hline $\begin{array}{c}\text { Sync } \\
(28)\end{array}$ & SACCH & Data & CDVCC & Data & RSVD \\
$(12)$ & $(130)$ & $(12)$ & $(130)$ & $(12)$ \\
\hline
\end{tabular}

Forward Link Burst (Base station to mobile station)

Data: User information

R: Ramp Time

G: Guard Time

SACCH: Slow Associated Control Channel

RSVD: Reserved (set to 0)

CDVCC: Coded Digital Verification Color Code

Figure 4.5: IS-54 time slot structure 


\subsection{Baseband software radio architecture for TDMA base station}

\subsubsection{Common modulator structure}

Two modulation schemes and the linear approximation of GMSK [37] are reviewed. A common modulator is proposed based on the approximation.

\subsubsection{Pi/4-DQPSK modulation scheme}

IS-54 use the $\frac{\pi}{4}-D Q P S K$ as its modulation scheme. The complex envelope of a $\frac{\pi}{4}-D Q P S K$ modulated signal is:

$$
\begin{array}{r}
S_{D Q P S K}(t)=\sum_{n=0}^{\infty} l_{n} \cdot g(t-n T) \\
\quad=\sum_{n=0}^{\infty} \exp [j \Phi(n)] \cdot g(t-n T)
\end{array}
$$

where $\mathrm{T}$ is the symbol duration and $l_{n}=\exp [j \Phi(n)]$ are complex symbols, whose phases are given as:

$$
\Phi(n)=\Phi(n-1)+\Delta \Phi(n)
$$

According to the principle of $\frac{\pi}{4}-D Q P S K$, at a time two bits are together assigned to one of four possible differential phases. Thus symbols $l_{n}$ are alternatively out of $\{-1,1,-\mathrm{j}, \mathrm{j}\}$ and $\left\{\frac{1}{\sqrt{2}}(1+j), \frac{1}{\sqrt{2}}(-1+j), \frac{1}{\sqrt{2}}(-1-j), \frac{1}{\sqrt{2}}(1-j)\right\}$. Information is placed into the differential phase of two successive symbols. Pulse former $\mathrm{g}(\mathrm{t})$ is a square root raised filter 
with roll off factor $\alpha=0.35$ for IS-54. In this case, Nyquist criteria are fulfilled and no inter-symbol interference (ISI) is produced by the filtering. The modulation can be realized with a I/Q-Modulator. Though theoretically the bandwidth efficiency is high, the modulation might have high amplitude fluctuation which leads to high requirements for the linearity of the end power amplifier.

\subsubsection{Linear approximation of GMSK modulation scheme}

Now we try to find an approximation of GMSK with a similar implementation structure with $\frac{\pi}{4}-D Q P S K$.

A GSM system using the nonlinear GMSK modulation scheme, is a special kind of a 2-level FSK (with modulation index $\mathrm{h}=0.5$ ) [60]. The complex envelope of a GMSK modulated signal is

$$
s(t)=\exp \left[j 2 \pi h \sum_{n=0}^{\infty} I_{n} \int_{-\infty}^{t} g(\tau-n T) d \tau\right]
$$

with the NRZ stream $I_{n} \in\{-1,1\}$ and the frequency impulse $\mathrm{g}(\mathrm{t})$.

In the GSM system, the bits $b_{i}$ are first differentially encoded

$$
\tilde{b}_{i}=b_{i} \oplus b_{i-1}, b_{-1}=1
$$

where $\oplus$ means modulo-2 addition. After that, the NRZ bitstream is built by transforming $\tilde{b}_{i}=1$ to $I_{i}=-1$ and $\tilde{b}_{i}=0$ to $I_{i}=1$.

To reduce the bandwidth, the following frequency impulse 


$$
g(t)=\frac{1}{2 T} \operatorname{rect}\left(\frac{t}{T}\right) \cdot h_{\text {Gauss }}(t)
$$

is used instead of a rectangular-impulse which is used at MSK. $h_{\text {Gauss }}(t)$ is the known Gaussian impulse with the time bandwidth product BT (filter bandwidth times bit period). The reduction of bandwidth is achieved with the trade of a controlled intersymbol interference (ISI). In GSM, the factor BT was chosen as 0.3, which results in a ISI over about 2 symbols but a small bandwidth. This ISI is equalized together with ISI caused by the mobile channel at the receiver.

The theoretically infinite long Gaussian impulse is realized by FIR-filter with the length cut to LT, where $L \geq 3$. Thus at time $\mathrm{t}=\mathrm{LT}$ the phase reply is

$$
q(t)=\int_{-\infty}^{t} g(\tau) d \tau
$$

With $\mathrm{q}(\mathrm{t})$, the GMSK signal $s(t)$ can be described as follows

$$
s(t)=\exp \left[j 2 \pi h \sum_{n=0}^{\infty} I_{n} q(t-n T)\right]
$$

The nth NRZ-bit $I_{n}$ causes a change of phase about $\pi q(t)$ or $-\pi q(t)$, which is added to the changes of the previous symbols. Here the information lies in the direction of the rotating complex signal vector. In [61], it is shown that $s(t)$ can be built by superposition of $N_{c}=2^{L-1}$ impulse $C_{K}$ :

$$
s(t)=\sum_{n=0}^{\infty} \sum_{K=0}^{N_{C}-1} \exp \left(j 2 \pi h A_{K, n}\right) C_{K}(t-n T)
$$


with

$$
\begin{gathered}
A_{K, n}=\sum_{i=0}^{n} I_{i}-\sum_{l=1}^{L-1} I_{n-l} \cdot \alpha_{K, l} \\
K=\sum_{l=1}^{L-1} 2^{l-1} \alpha_{\dot{K}, l}
\end{gathered}
$$

where $\alpha_{K, l} \in\{0 ; 1\} . \mathrm{C}_{\mathrm{k}}$ are a serial amplitude modulated pulses obtained in [61]. For GMSK with $\mathrm{L}=4$, the exact superposition is made by eight impulses [62].

The approximation of the GMSK signal $s(t)$ is then given as

$$
\begin{aligned}
s(t) & \cong \sum_{n=0}^{\infty} \exp \left[j \pi h \sum_{i=0}^{\infty} I_{i}\right] C_{0}(t-n T) \\
& =\sum_{n=0}^{\infty} z_{n} C_{0}(t-n T)
\end{aligned}
$$

where $z_{n} \in\{-1,1,-j, j\}$. Compared with equation (4.1), the approximated GMSK-signal can be built similarly to the $\frac{\pi}{4}-D Q P S K$ signal. That means $\mathrm{s}(\mathrm{t})$ is formed by impulse symbols $C_{0}(t)$ multiplied by $z_{n}$ which is determined from $I_{i}$ accumulation. Thus this approximated GMSK can be realized with a usual I/Q-modulator for PSK or QAM and is therefore easy to integrate into a software radio architecture.

The approximation of the GMSK will cause a certain performance loss as discussed in [62]. However, the power spectrum density of the approximated GMSK fits the 


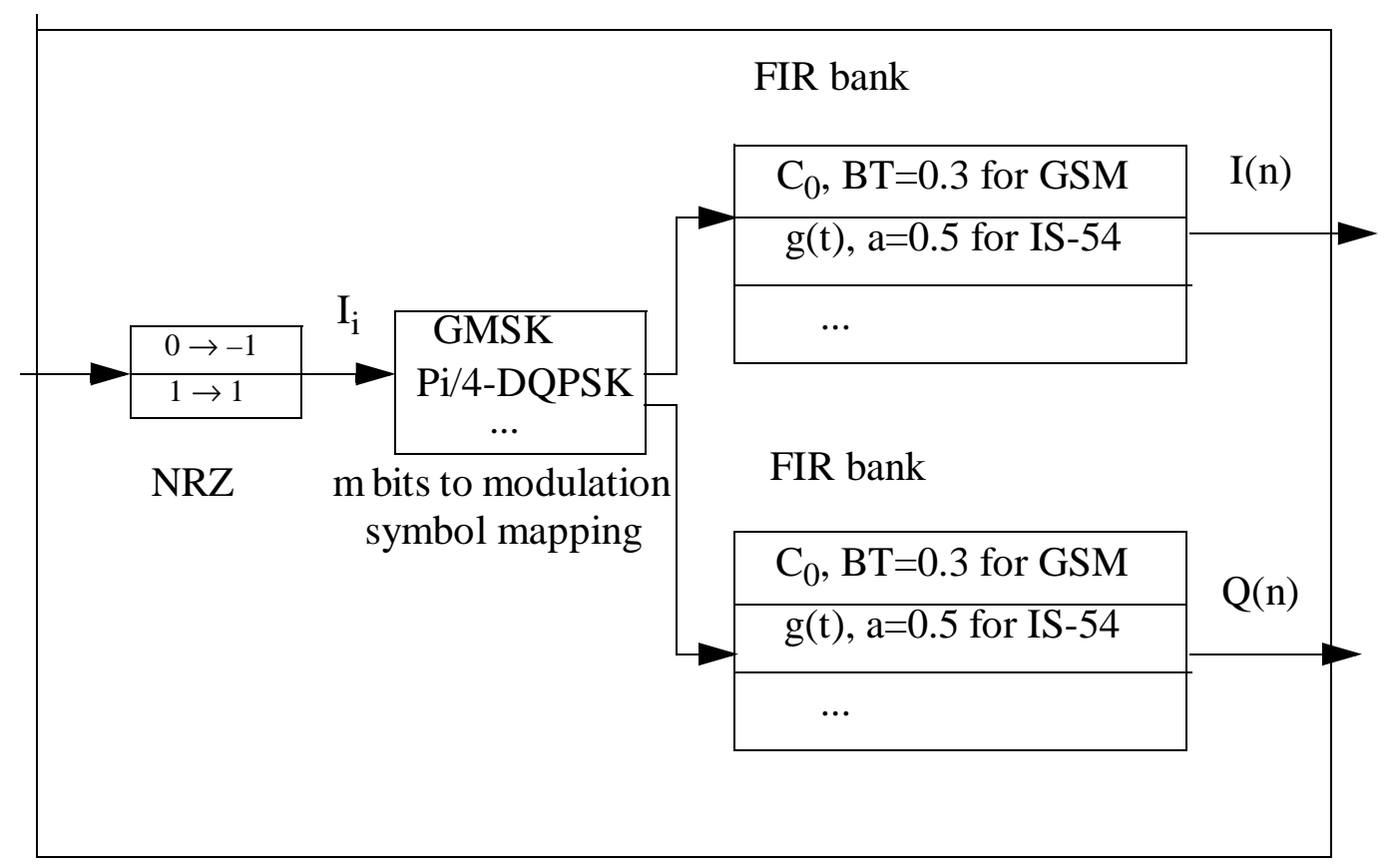

Figure 4.6: One Common I/Q-modulator with different system parameters

actual one very well. This means the linear approximation does not change the signal significantly.

One common modulator structure, Figure 4.6, is proposed for GSM and IS-54. 


\subsubsection{One possible common demodulator architecture}

We found a feasible common baseband transmitter above. How about the receiver?

In mobile communication environments, the propagation channel is frequency selective. Hence, for broadband systems, the intersymbol interference (ISI) occurs during the transmission. The number of overlapped symbols depends on the symbol duration $\mathrm{T}$ of different cellular systems and the maximum path delay $\tau_{\max }$ of the channel assumed.

For a GSM system with symbol duration $T=3.69 \mu s$ (see Figure 4.2), if we assume the maximum path delay is $\tau_{\max }=20 \mu s$ [63], the ISI is about 6 symbols. For IS-54, due to a more efficient speech encoder and the four-level modulation scheme, the symbol duration $\mathrm{T}$ is $41.14 \mu$ s (See Figure 4.5. The sampled signal is sent out in a compressed time frame over ten interleaved time slots). If the same maximum path delay is assumed, a possible ISI occurs between two successive symbols.

Therefore, in the proposed software radio common receiver structure for GSM and IS-54, a general flexible Viterbi-equalizer is needed.

\subsubsection{Common equalizer}

The general Viterbi equalizer require the following main parameters [37]: the discrete channel model with constraint length $\mathrm{L}$, the number of the modulation symbols and the possible transition states, and the number of symbols of one block.

The discrete channel model with constraint length L can be described by a trellis

diagram. The total number of states is $N^{L-1}$, where $\mathrm{N}$ is the possible symbol number at the same time. 
The trellis diagram is different for the various mobile systems. For IS-54 L=2 and Table 4.1: Parameters relating to the proposed common viterbi equalizer

\begin{tabular}{|c|c|c|}
\hline & GSM & IS-54 \\
\hline \hline $\begin{array}{c}\text { constraint length L of correspond- } \\
\text { ing discrete channel model }\end{array}$ & 7 (normally 3, 4) & 2 \\
\hline Modulation scheme & GMSK & $\pi / 4-D Q P S K$ \\
\hline $\begin{array}{c}\text { Number of symbols N at same } \\
\text { time according to different modu- } \\
\text { lation schemes }\end{array}$ & 2 & 4 \\
\hline Total number of states $N^{L}$ & 128 (normally 16, 32) & 4 \\
\hline Channel estimation method & Training sequence & $\begin{array}{c}\text { Synchronization } \\
\text { sequence (26 bits) }\end{array}$ \\
\hline
\end{tabular}

$\mathrm{N}=4$. Thus, the number of states is four. For GSM, the ISI calculation leads to a channel model with constraint length $\mathrm{L}$ of about 7 , so the total states is 128 , which would need too much calculation and in most applications 16 or 32 states are normally used [37].

For the Viterbi algorithm in both GSM and IS-54 systems, MLSE equalization can be applied. For MLSE, the channel impulse response must be estimated. In GSM, the burst length is $0.577 \mathrm{~ms}$ (Figure 4.3); the channel response can be assumed to be stationary during one GSM burst. The channel estimation can be reduced to one per burst. The IS-54 burst (Figure 4.5) is $6.67 \mathrm{~ms}$ long and therefore channel tracking is necessary. A known training or synchronization is used for the channel estimation. In the GSM burst, this sequence is placed in the middle. The estimation is performed by correlation of the received training sequence with the known training sequence.

Based on the above analysis, one common equalizer is proposed in Figure 4.7. Still further study is needed for the more detailed design for each function block. 

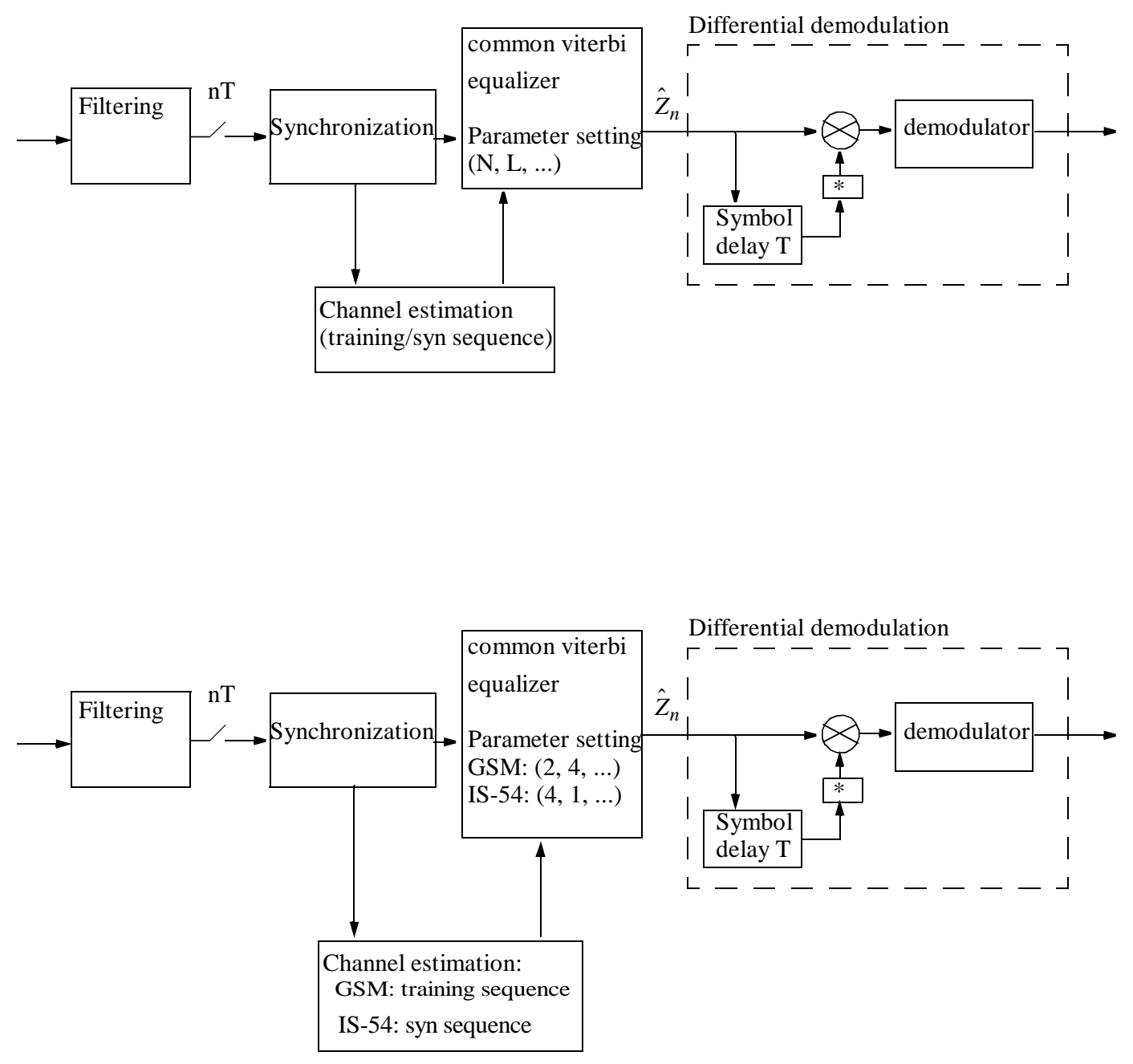

Figure 4.7: One common receiver structure for GSM and IS-54 


\subsection{Parameterized structure for channel coding/interleaving}

Based on the baseband function review, we now can get the parameterized functional block for channel coding and interleaving for both the IS-54 and GSM systems.

The convolutional channel coding can be described by the generator polynomials (in binary representation), the code rate (which is not always equal to the number of generator polynomials), and the constraint length. In a similar way, interleaver can be presented by the two parameters, which describe the interleaving matrix.

Table 4.2 and Table 4.3 gives the main parameter set summary of channel coding and interleaving functional blocks in both GSM and IS-54 systems.

Table 4.2: The main channel coding parameter for GSM and IS-54

\begin{tabular}{|c|c|c|c|}
\hline Channel coding parameter & $\mathrm{R}$ & $\mathrm{K}$ & $\mathrm{G}$ \\
\hline \hline GSM & $1 / 2$ & 5 & $\mathrm{G} 0(\mathrm{X})=\mathrm{X}^{3}+\mathrm{X}+1$ \\
$\mathrm{G} 1(\mathrm{X})=\mathrm{X}^{4}+\mathrm{X}^{3}+1$ \\
$\mathrm{G} 2(\mathrm{X})=\mathrm{X}^{4}+\mathrm{X}^{3}+\mathrm{X}+1$
\end{tabular}

Table 4.3: The main interleaving parameter for GSM and IS-54

\begin{tabular}{|c|c|}
\hline Interleaving parameter & $(\mathrm{X}, \mathrm{Y})$ \\
\hline \hline GSM & $\mathrm{S}:(8,57)$ \\
\hline IS-54 & $\mathrm{S}:(10,50)$ \\
\hline
\end{tabular}


The parameterized functional blocks for channel coding and interleaving are given in Figure 4.8 and Figure 4.9 respectively.

Similar parameterized functions can be formed for the burst builder and multiplexer according to the detailed specifications for the two systems.

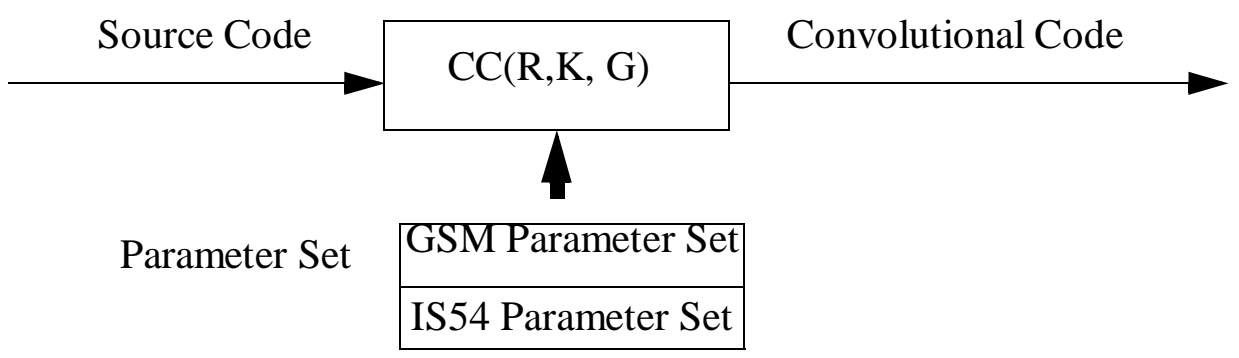

GSM Parameter Set

$\mathrm{R}=1 / 2 ; \mathrm{K}=5$
$\mathrm{G} 0(\mathrm{X})=\mathrm{X}^{3}+\mathrm{X}+1$
$\mathrm{G} 1(\mathrm{X})=\mathrm{X}^{4}+\mathrm{X}^{3}+1$
$\mathrm{G} 2(\mathrm{X})=\mathrm{X}^{4}+\mathrm{X}^{3}+\mathrm{X}+1$

IS54 Parameter Set

$\mathrm{R}=1 / 2 ; \mathrm{K}=6$
$\mathrm{G}=\mathrm{X}^{7}+\mathrm{X}^{5}+\mathrm{X}^{4}+\mathrm{X}^{2}+\mathrm{X}+1$




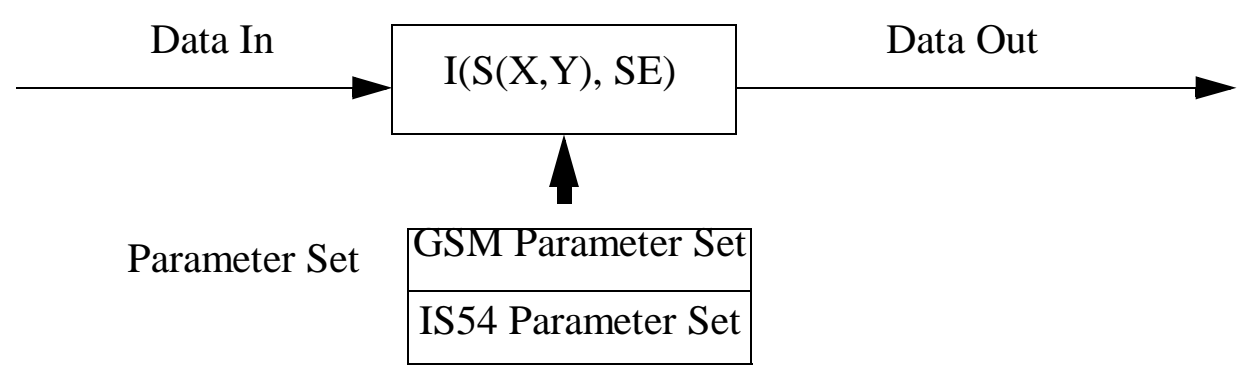

GSM Parameter Set

IS54 Parameter Set

$\mathrm{S}:(8,57)$

$\mathrm{S}:(10,50)$

Figure 4.9: Parameterized structure for interleaving 


\subsection{SWR architecture for the baseband processing of main function}

Based on the above analysis, we may get the parameterized architecture for the

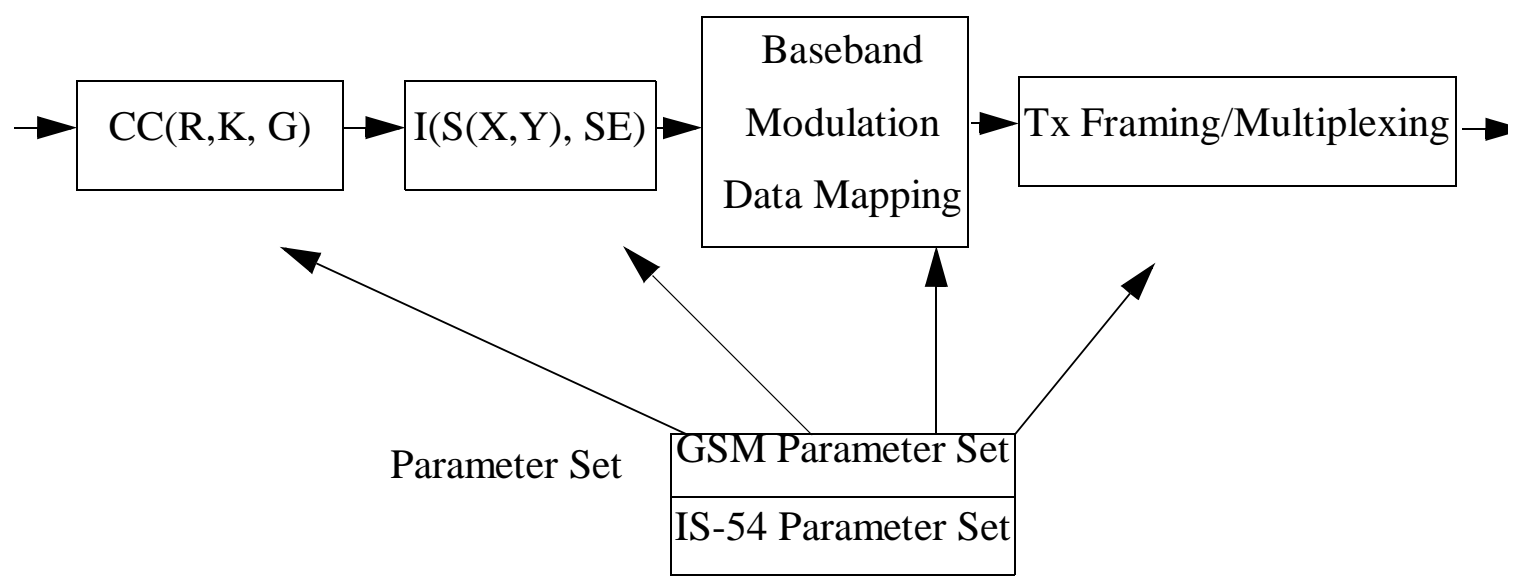

Figure 4.10:Parameterized architecture for baseband processing

base station (see Figure 4.10). Considering the smart antenna application, the integrated solution is given in Figure 4.11.

Figure 4.10 and Figure 4.11 only give a basic structure of the design of baseband processing. Still further study is needed to complete the detailed design of the proposed architecture. Parameterized method should be applied to other part of functions, such as the general burst builder and multiplexer. More considerations should be given to technical issues such as parameter swapping. 


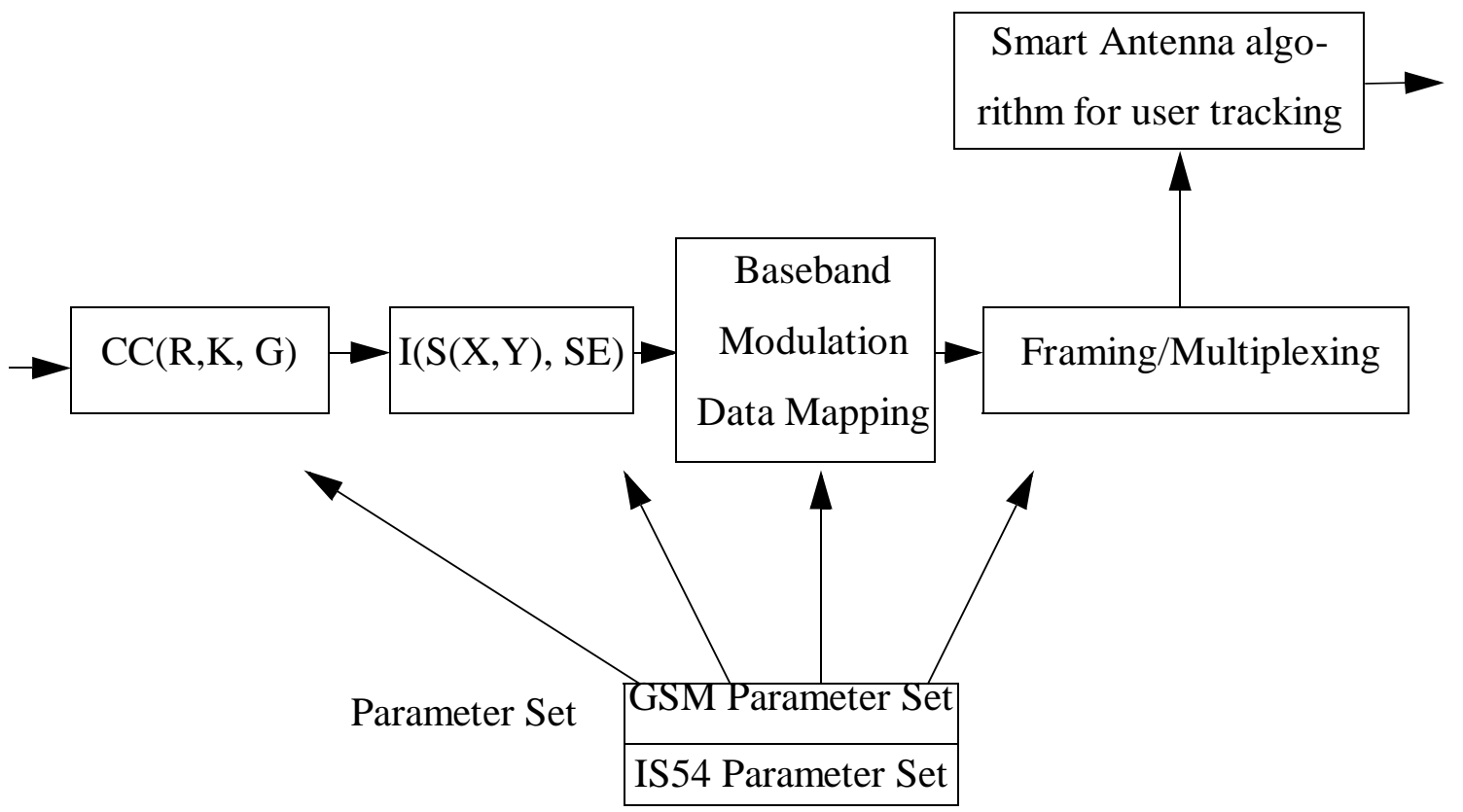

Figure 4.11: Parameterized functional structure for baseband processing with smart antenna 


\section{Chapter 5}

\section{Smart Antenna Integration in SWR Base Station Architecture}

This chapter is organized as follows: adaptive beam smart antennas and beamforming algorithms are first reviewed. The evolution of the software radio architecture for the base station is described. Functional block diagrams of the software radio base station with smart antennas are proposed and described. One possible implementation using DSP and FPGA and related channel assignment algorithms for the proposed software radio architecture are presented. Limitations and further study of the architecture are given. In addition, the beamforming algorithm of the smart antenna and the influence of its integration in GSM are also addressed.

In this Chapter, adaptive beam antennas are used in the analysis.

\subsection{Smart antenna and beamforming algorithms review}

\subsubsection{Adaptive beam smart antenna}

The block diagram of an adaptive array with M elements is depicted in Figure 5.1, where the gain of each antenna element is weighted by complex weights $\omega_{i 1}, \omega_{i 2}, \omega_{i 3}, \ldots$, $\omega_{i M}$. By adaptively adjusting the weights of the antenna array, some appropriate antenna patterns can be formed according to the temporal or spatial signature, such as DOA's of the interference signals or/and statistical nature of desired signals. This adaptive beamforming capability makes it possible for the antenna arrays to maintain a constant gain for 
the signal along the direction of interest, while adjusting the nulls so as to reject the cochannel interference. In this way, the co-channel interference (CCI) is minimized and the CIR for the signal of interest is maximized.

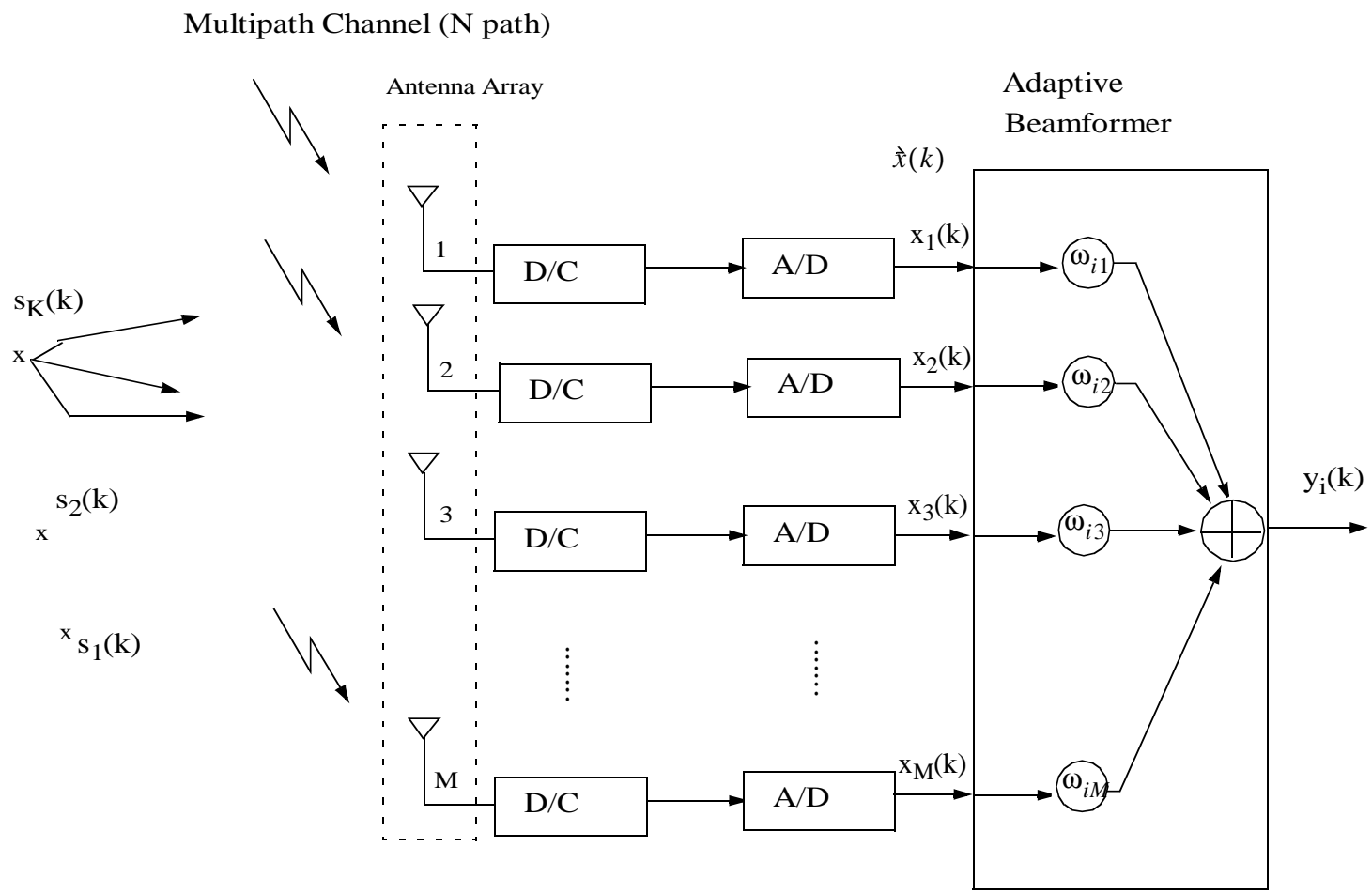

D/C: RF down converter

A/D: A/D converter

$\mathrm{K}$ : Cochannel transmitter number

N: Maximum possible multipath

M: Element number of smart antenna array

Figure 5.1 An adaptive array system with space diversity combiner 


\subsubsection{Spatial/temporal adaptive beamforming methods}

In Figure 5.1, a set of K co-channel transmitters is considered. Each transmits signal denoted by $s_{j}(k)$ with $\mathrm{j}=1,2, \ldots, K$. Assume that the number of multipaths for each transmitted signal is $N$ and each arrives in the base station with different delays $\tau_{n}$. Therefore, the total received signal vector corresponding to the $s_{j}(k)$ is:

$$
\vec{x}(k)=\sum_{j=1}^{K} \sqrt{P_{j} G_{j}} \sum_{n=1}^{N} \alpha_{j n} \vec{a}\left(\theta_{j n}\right) s_{j}\left(t-\tau_{j n}\right)+\vec{n}(k)
$$

where $\theta_{j n}$ is the arrival direction of the nth multipath signal from the jth user, $P_{j}$ is the power of the jth transmitter, $G_{j}$ is the link gain between the jth transmitter and base station, $\alpha_{j n}$ is the nth path fading coefficient for jth user, and $\vec{a}\left(\theta_{j n}\right)$ is the array response to the multipath signal arriving from direction $\theta_{j n}$ with:

$$
\vec{a}\left(\theta_{j n}\right)=\left[a_{1}\left(\theta_{j n}\right), a_{2}\left(\theta_{j n}\right), \ldots, a_{M}\left(\theta_{j n}\right)\right]^{T}
$$

There are two main adaptive beamforming methods: the space diversity combining and the space-time diversity combining.

\subsubsection{Space diversity combining}

Figure 5.1 shows a diagram for a space diversity system, where the received signal at each element is weighted and combined at the output of the beamformer. The beamformer output is a weighted sum of the array elements: 


$$
y_{i}(k)=\vec{\omega}_{i}^{H}{ }_{\vec{x}}(k)
$$

where $\vec{\omega}_{i}$ is the weight and $\vec{X}^{T}(k)$ is the sampled received signal vector.

There are two main algorithm catalogs for space diversity combining:

- Minimum variance distortionless response (MVDR)[57]:

Using MVDR, the weight vector is chosen to maintain a distortionless response in the direction of interest, while placing nulls in the directions of other co-channel interferers. If the array response in the direction of the desired user is known, the beamformer tries to minimize the output power $\mathrm{E}_{\mathrm{i}}$ but maintain a distortionless response in the direction of interest. The average output power is:

$$
E_{i}=E\left[y_{i}(k) y_{i}{ }^{H}(k)\right]={\omega_{i}}^{H} E\left[\vec{x}(k) \vec{x}^{H}(k)\right] \omega_{i}={\omega_{i}}^{H} \Phi \omega_{i}
$$

The optimum weight vector minimizes the interference while maintaining a unity gain in the direction of interest by imposing $\omega_{i}^{H} \vec{a}_{i}\left(\theta_{i i}\right)=1$. The solution to this problem is given in [57].

$$
\hat{\omega}_{i}=\frac{\Phi^{-1} \vec{a}_{i}\left(\theta_{i 1}\right)}{\vec{a}_{i}^{H}\left(\theta_{i 1}\right) \Phi^{-1} \vec{a}_{i}\left(\theta_{i 1}\right)}
$$

- Minimum Mean-Square Error (MMSE)[57]:

For MMSE, the array response is unknown. A training sequence is needed to minimize the difference between the known training sequence $d_{i}$ and the output of the beamformer in the mean square sense. 


$$
\hat{\omega}_{i}=\underset{\omega_{i}}{\arg } E\left[\left|d_{i}-\omega_{i}^{H} \vec{a}_{i}\left(\theta_{i}^{1}\right)\right|^{2}\right]
$$

The solution to this problem is given in [57]

$$
\hat{\omega}_{i}=\Phi^{-1} \vec{p}_{i}
$$

where $\Phi$ is the same as in equation (5.4) and $\vec{p}_{i}$ is the cross correlation between the received vector and the training sequence.

\subsubsection{Space-time diversity combining}

In the space diversity combining system, the interference and multipath signals are rejected by placing nulls at the directions of those signals. In the space-time diversity combiner, the desired signal and its multipath are combined at the combiner output to estimate the desired signal. There are two groups: MMSE space-time combining [20], such as recursive least-square (RLS) [20] and minimum mean-square error (MMSE).

\subsubsection{Smart antenna beamforming}

The beamforming(BF) algorithm can be classified as [58]: spatial structure based beamforming such as direction of arrival (DOA) based beamformer (DOB), training signal based beamformer such as temporal reference BF (TRB), which use adaptive algorithms such as LMS, RLS, and signal structure based BF (SSBF) which exploit temporal and/or spectral properties of the received signals

Table 5.1 gives the comparison of the three.

Table 5.1: Smart antenna processing algorithms

\begin{tabular}{c|l}
\hline \multicolumn{1}{c|}{ Beam former (BF) types } & \multicolumn{1}{c}{ Description } \\
\hline Direction of arrival (DOA) based BF & $\begin{array}{l}\text { - DOA estimation method is needed } \\
\text { - apply when angular spread of the received signals is rel- } \\
\text { atively small }\end{array}$ \\
\hline
\end{tabular}


Table 5.1: Smart antenna processing algorithms

\begin{tabular}{l|l}
\multicolumn{1}{c|}{ Beam former (BF) types } & \multicolumn{1}{c}{ Description } \\
\hline $\begin{array}{l}\text { Time reference (training signal) beamforming } \\
\text { (TRB) based BF }\end{array}$ & $\begin{array}{l}\text { + good fit for rich multipath environment } \\
\text { - reference-signal-based, spectrum efficiency is reduced }\end{array}$ \\
\hline Signal structure based BF(SSB) & + if no reference signal, difficult to obtain \\
\hline
\end{tabular}

\subsection{Beamforming of smart antennas in GSM}

The smart antenna algorithm chosen for GSM is dependent on the environment. In a macrocell with low traffic, there is more noise than co-channel interference (CCI), high user mobility and low angular spread. The preferred algorithm is a direction-of-arrival based-beam-former (DOB), whose performance depends on the ratio of angular spread with the number of antenna elements M. For a microcell with high traffic, there is high CCI, low user mobility, high angular spread and low delay spread, so time-referencebased beamforming (TRB, such as LMS, RLS, DMI etc.) is suitable. The colored training sequence is used for time reference in the GSM system.

The integration of smart antenna technology will influence the first three layers of the protocols reference model in the GSM system. Several main procedures in the GSM system need to be changed for the integration such as: log-in procedure, handover signaling, link quality monitoring, frequency hopping, location updating, and time advancing procedure. In addition, the following issues need further research, such as beams collision avoidance, physical link control algorithms, performance and compatibility, and resources management issues. The influence is summarized in Table 5.3.

There are still many issues, relating to the detailed implementation design of smart antenna algorithm in GSM basestation, that need further study. These issues include: the dynamic range of the receive GSM signals and weight coefficients, the timing of the 
beamforming operation, the sampling rate at which beamforming is performed, which affect the requirement of processing power of hardware such as DSP and/or FPGA.

Table 5.2: Smart antenna beamforming algorithms

\begin{tabular}{l|l}
\hline \multicolumn{1}{c|}{ Cell types } & \multicolumn{1}{c}{ Algorithms suggested } \\
\hline $\begin{array}{l}\text { Macrocell } \\
\text { low traffic } \\
\text { more noise than CCI environment } \\
\text { high user mobility } \\
\text { low angular spread }\end{array}$ & $\begin{array}{l}\text { DOB: performance depends on the ratio } \\
\text { of angular spread with the number of } \\
\text { antenna elements M }\end{array}$ \\
\hline $\begin{array}{l}\text { Microcell } \\
\text { high traffic } \\
\text { high CCI } \\
\text { low user mobility } \\
\text { high angular spread } \\
\text { low delay spread }\end{array}$ & $\begin{array}{l}\text { TRB: (colored training sequence in GSM } \\
\text { is used) }\end{array}$ \\
\hline
\end{tabular}

Table 5.3: Issues related to smart antenna integration

\begin{tabular}{l|l}
\hline $\begin{array}{c}\text { Main procedures } \\
\text { need to be changed }\end{array}$ & Several issues need further research \\
\hline $\begin{array}{l}\text { log-in procedure, } \\
\text { handover signal- } \\
\text { ing, link quality } \\
\text { monitoring, fre- } \\
\text { quency hopping, } \\
\text { location updating, } \\
\text { time advancing } \\
\text { procedure }\end{array}$ & $\begin{array}{l}\text { Beams collision avoidance in SFIR (color codes and intracell handover) } \\
\text { smart antenna (Performance and dynamics of PC and SA tracking algo- } \\
\text { rithms) } \\
\text { Resources management (optimisation of BS assignment, channel alloca- } \\
\text { tion, beamformer and power control algorithms at the up- and down-link) }\end{array}$ \\
\hline
\end{tabular}




\subsection{Base station software radio architecture evolution}

There is a wide gulf between the ideal software radio and the pragmatic reality of commercially available technology today. Figure 5.2 shows one pragmatic approach for the software radio base station evolution.

Phase I architecture implements channel coding, and source coding in software on DSP. It allows reconfiguration of the application layer, such as new service introduction.

The extension of the above is to implement the baseband modem functionality in software shown in phase II, which allows the realization of new and adaptive modulation schemes.

Further extension to implement the intermediate frequency (IF) signal processing in software will allow a base station to adapt to multiple radio interface standards by software reconfigurability. It involves the reconfigurability at the lowest level of the protocol stack.

The software radio architecture proposed here is between Phase I and Phase II. Both the baseband modulation and the multistandard support are considered. 


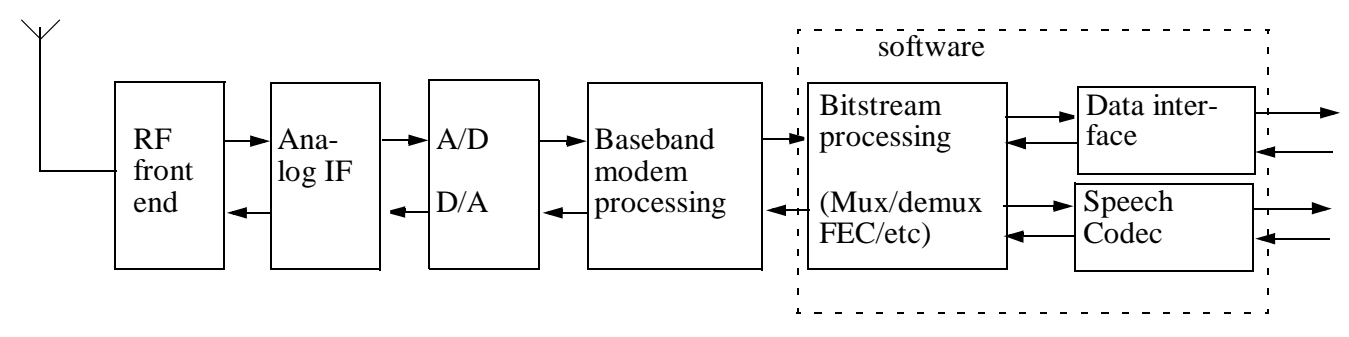

PHASE I

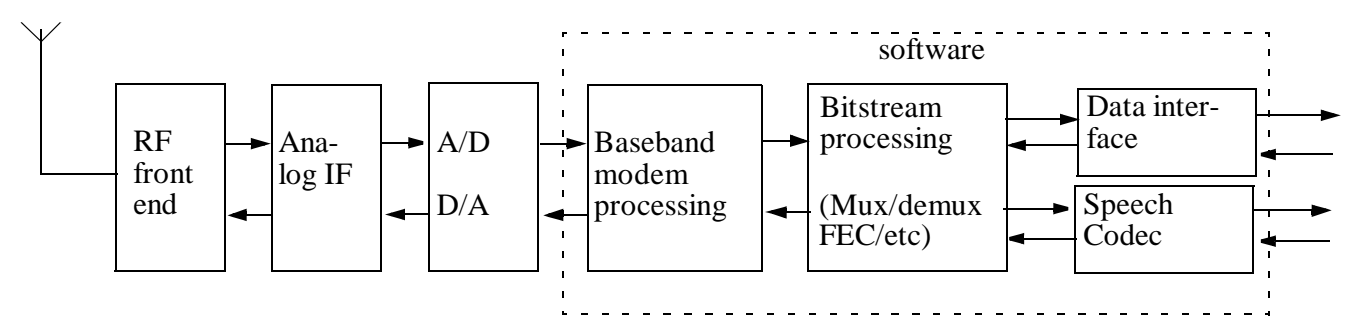

PHASE II

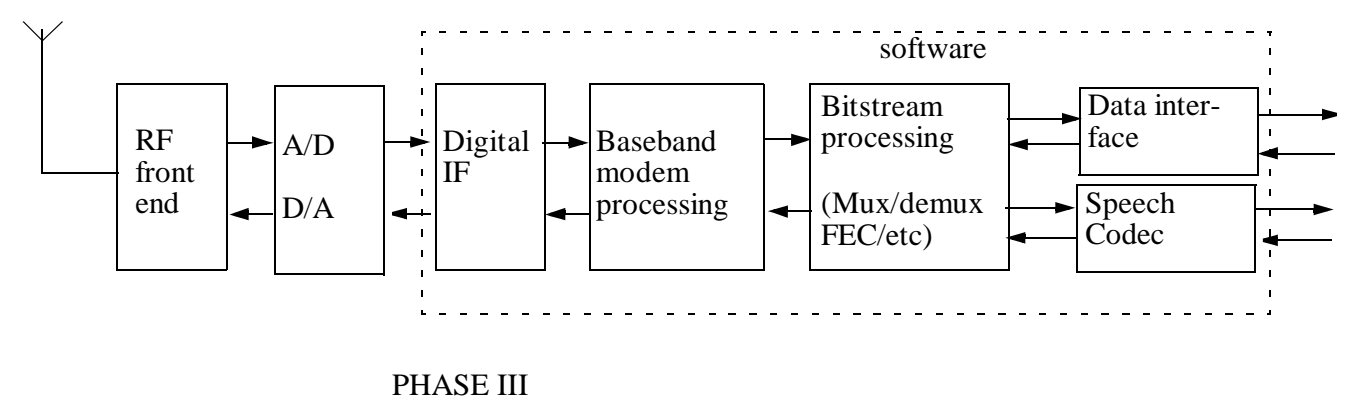

Figure 5.2 Possible software radio architecture evolution for base station 


\subsection{Functional block diagram of the SWR base station with smart antennas}

The software radio base station architecture with smart antenna proposed here will serve as a good example to illustrate the great flexibility of software radio. In this architecture, the smart antenna algorithms can be dynamically reconfigured according to different environment requirements and the baseband processing can be dynamically reconfigured according to different standards requirements.

Figure 5.3 shows the function block diagram of the software radio base station with smart antennas. Each antenna element has its own down converter and ADC. K similar blocks are needed for the subsequent beamforming and demodulation, which are implemented in software.

The beam formers for each channel is illustrated in Figure 5.4. The digital downconverter includes the function of frequency translation and filtering. The received IF signals from each antenna element are translated to a complex baseband signal by the quadrature multiplier. The digitally controlled oscillator (DCO) block generates the quadrature signals for the multipliers. Then the complex baseband signal is low-passed filtered. Finally these signals are adjusted by the weights generated by the smart antenna algorithms in beam former, and combined in the "combiner" block. The output of the demodulator would be the received signal from the desired user.

Here, we assume each channel can be shared by K users. K beams are formed, one for each user. Thus, for the system, shown in Figure 5.2 and Figure 5.4, with L physical channels, KL users can be supported.

The smart antenna algorithm chosen to adjust the weights is dependent on the environment. For GSM system, in macrocell with low traffic, more noise than cochannel interference $(\mathrm{CCI})$, high user mobility and low angular spread, the preferred algorithm is 
Direction of arrival based beam former (DOB), whose performance depends on the ration of angular spread with the number of antenna elements M. For the microcell with high traffic, high CCI, low user mobility, high angular spread and low delay spread, time reference based beamforming is suitable. The software implementation of the beam former provides the reconfiguration flexibility for the algorithms chosen according to the environment requirement.

The baseband processing functions are implemented on a common platform using a parameterized implementation method, which enables the air interface to be changed fast by only exchanging a set of parameters for the reconfiguration, rather than download the whole software of a system. Figure 5.3 illustrates such a common baseband processing platform for GSM and IS-54 TDMA systems. In both of the systems, main functions like channel coding, modulation and equalization, are implemented using a parameterized method on one common transceiver architecture.

The proposed architecture provides the flexibility for smart antenna algorithms to be reconfigured according to different environment requirements and for baseband processing functions to be reconfigured according to different standards requirements.

Figure 5.5 give one example for an adaptive algorithm in the beam-former. Here the interference cancellation algorithm is proposed by IAI Company [59] and implemented by software. The program consists of four parts: (a) Preprocessing (this is not necessary if the desired signal and the jammers are non-coherent); (b) adaptive principal component analysis (PCA) to extract the principal eigenvectors which span the signal subspace; (c) a 1-dimensional search algorithm to locate the directions of arrival of the interference sources; (d) an off-line trained Fuzzy CMAC [59] neural network which learns the nonlinear relationship between the DOAs, hopping frequencies, and the desired weights of the antenna array elements. One advantage of using Fuzzy CMAC is that the geometry of the phased array does not need to be linear, i.e. irregular array patterns can be dealt with. The above beamforming process can be implemented on DSPs. The system makes use of 
two beamformers. The beamformer on the right is connected directly to the elements and is used to derive the array output signal. The beamformer on the left is the adaptive beamformer.

Analysis [59] shows that the proposed method can achieve high interference signal rejection within $0.1 \mathrm{~ms}$ whereas other methods such as LMS, take a long time to reach steady-state. That means the proposed method achieves faster convergence than the LMS method. In addition, the proposed method can meet the requirement of frequency hopping systems for different DOAs. 


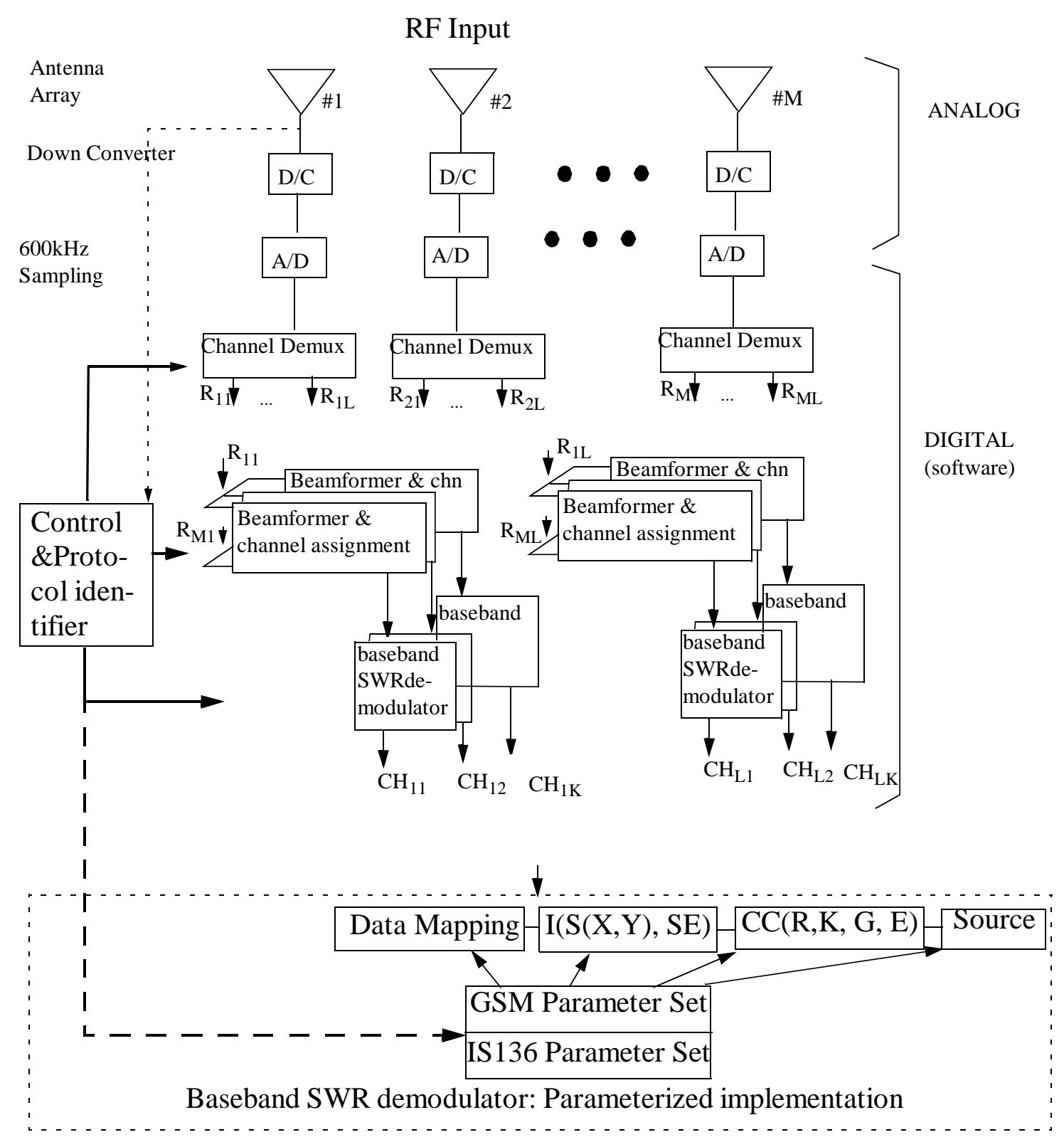

M: element number of smart antenna

$\mathrm{K}$ : user number sharing the same channel

L: Channel number

Figure 5.3 Functional block diagram of the software radio for a multimode base station with smart antenna 
IF signal from each antenna element

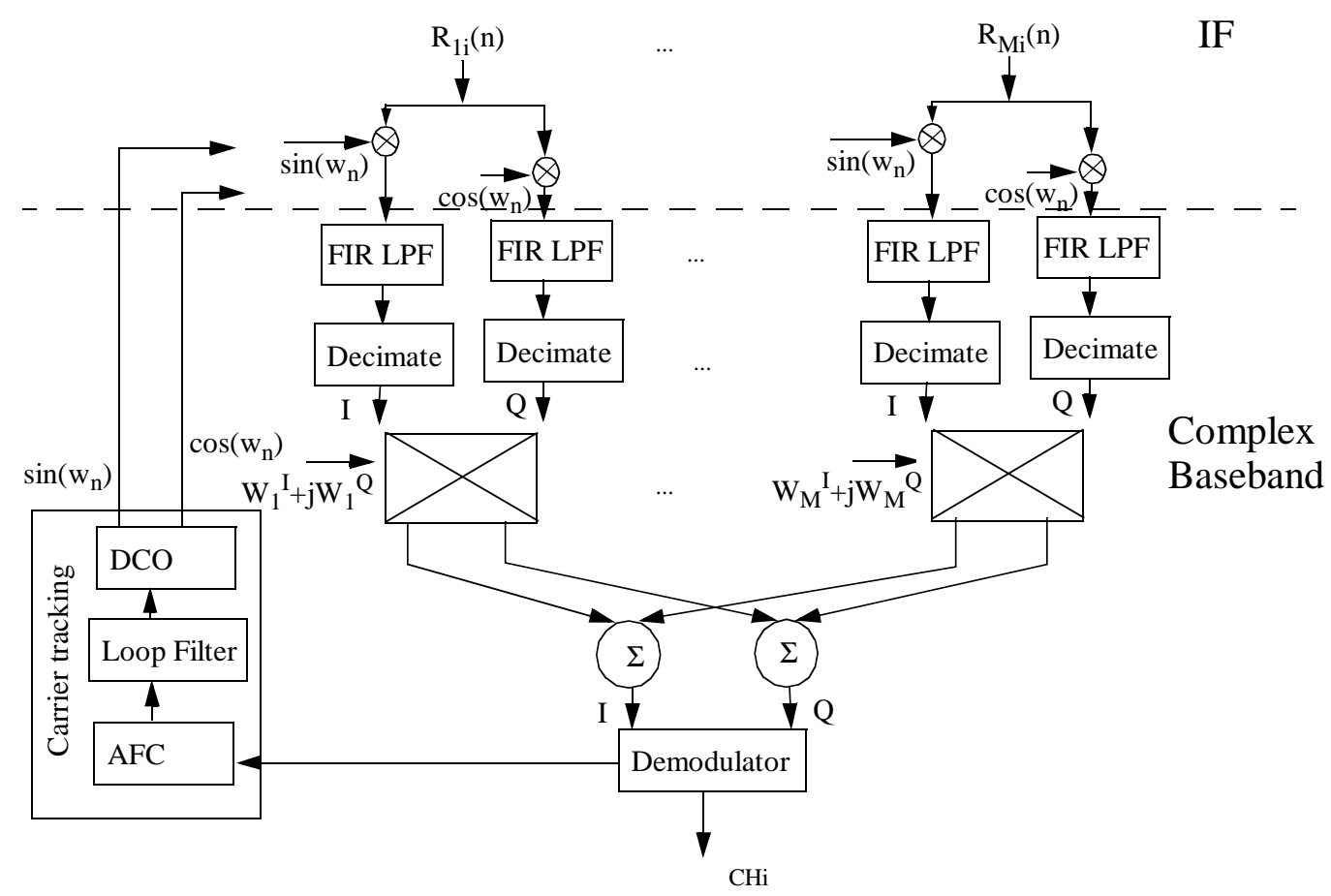

M: element number of smart antenna

$\mathrm{K}$ : user number sharing the same channel

L: Channel number

Figure 5.4 Diagram of beamformer for each channel 


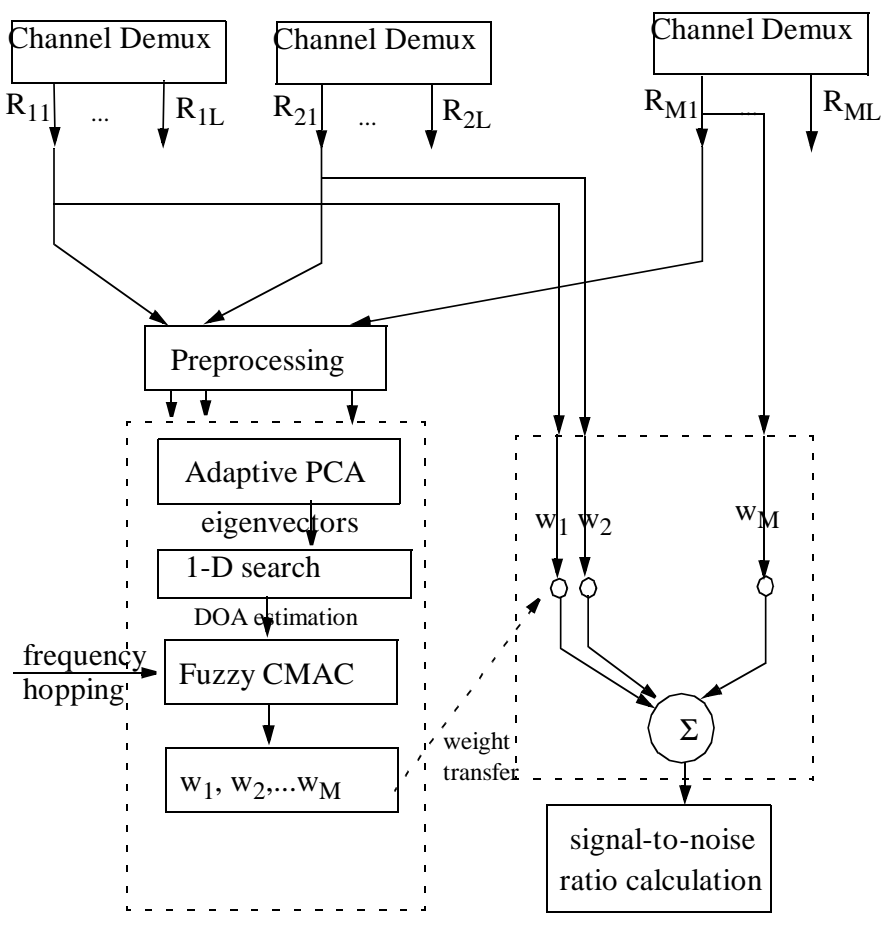

M: element number of smart antenna

$\mathrm{K}$ : user number sharing the same channel

L: Channel number

Figure 5.5 Diagram of possible weights generator for the beam former 


\subsection{Possible architecture using DSP and FPGA}

\subsubsection{Possible architecture using DSP and FPGA}

Figure 5.6 and Figure 5.7 give a feasible architecture for the software radio base station using DSP and FPGA technologies.

Here, GSM and/or IS-136 band are selected out of full RF band by means of analog conversion and IF filtering. The architecture includes: channel processing (IF to baseband translation, low pass filtering), estimation processing (estimation of signal and interference parameters and correlations, beam forming), matched filtering and information bit stream processing.

The main functionality are partitioned into two core technologies (field programmable gate arrays (FPGA) and digital signal processor (DSP) devices) based on processing ability requirements, considering the extension of the architectures to support higher bandwidth.

FPGA can be used to realize a signal processing platform that can run at relatively high clock rate and can be reconfigured completely. So we use FPGA for the channel processing and estimation processing and use DSP for the control and bit stream processing. 


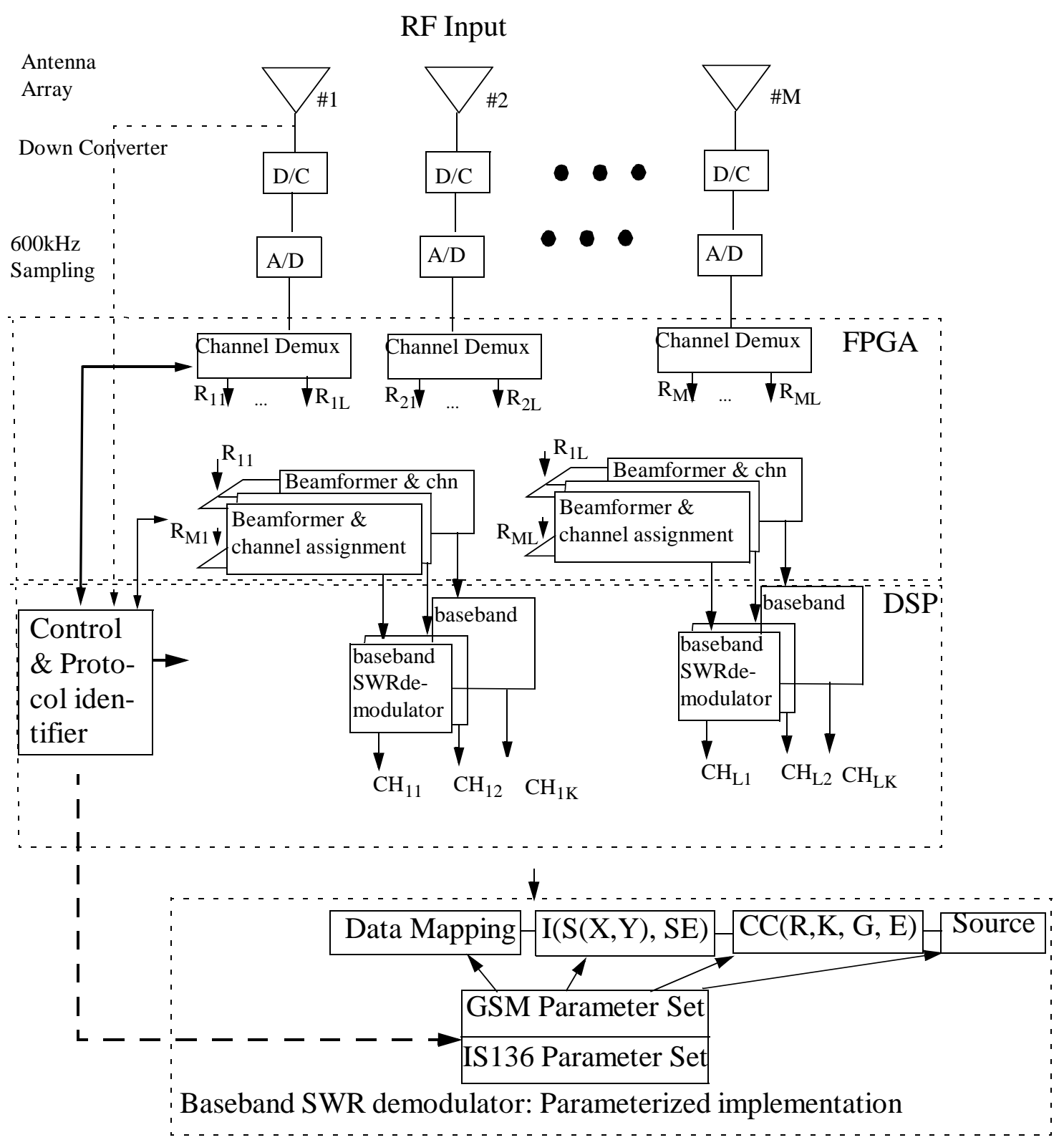

M: element number of smart antenna

$\mathrm{K}$ : user number sharing the same channel

L: Channel number

Figure 5.6 General functional architecture for software radio base station with smart antenna 


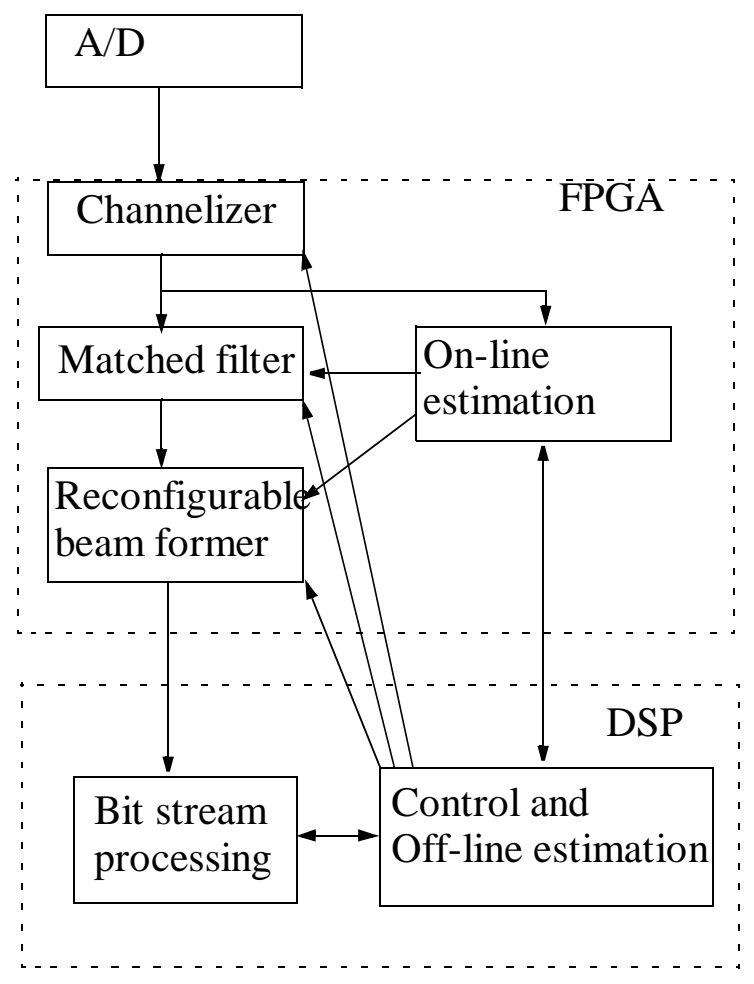

Figure 5.7 Functional architecture for software radio base station with smart antenna 


\subsubsection{Advantages and limitations}

The proposed software radio base station provides the reconfiguration flexibility to support multiple mobile system standards. It also offers substantial system performance and implementation simplification when integrated with smart antenna. However, ideal software radio architecture poses high requirements for the linearity of wideband and dynamic range for A/D convertor. In order to support the wideband receiver, further studies are needed, such as:

- The evolution of RF semiconductor process and A/D, D/A convertor technologies is expected to satisfy the high dynamic range requirements for $\mathrm{A} / \mathrm{D}, \mathrm{D} / \mathrm{A}$ convertor, and the high linearity for wideband RF processing.

- Baseband DSP architecture for IS-136/GSM needs to be extended to support more standards, such as UMTS.

- Further combination with other advanced processing functions is needed. These functions include: recognitions of incoming transmission mode, adaptive interference mitigation, adaptive channel estimation and equalization.

- Simulation is needed for the proposed software radio base station architecture. SPW's signal flow simulator is suggested for the simulation.

\subsection{Channel assignment algorithm in GSM system with smart antenna}

The discussion here is based on the proposed architecture as shown in Figure 5.3 and Figure 5.6, where $\mathrm{M}$ is the number of smart antenna elements, $\mathrm{L}$ is the total number of 
RF frequency channel and $\mathrm{K}$ is the total number of users sharing the same channel. Here, we assume that when a new call (handoff or originating call) arrives, the adaptive array points one beam toward that user, and assigns one channel out of those $\mathrm{L}$ channels to that user. Each channel $C h_{i}$ for $\mathrm{i}=1,2, \ldots, \mathrm{L}$ may be assigned to one of the $\mathrm{K}$ users by $\mathrm{K}$ separate beams, using the $\mathrm{K}$ beamformers in parallel for each channel, as shown in Figure 5.2 and Figure 5.3. If the first beam of all channels is occupied, the new call is assigned to another beam. If there are no multipaths in the system, with $\mathrm{K}$ beamformers for each channel and L RF frequency channels, at most KL users can be accepted into the system. On the other hand, if there are multipath signals with effective $\alpha$ paths per user, then the number of antennas has to increase to null those multipaths if a space-only diversity combining algorithm is applied. In this case, the effective number of usable channels would be $K L / \alpha$. The flowchart of the channel assignment with smart antennas is illustrated in Figure 5.8.

For a newly arrived ith mobile station, assuming that $(\mathrm{i}-1)<\mathrm{K}$ co-channel transmitters have successfully shared the same channel, it shares that channel if $\Gamma_{i} \geq \gamma$, where $\Gamma_{i}$ is the carrier-to-interference ratio and $\gamma$ is a system parameter which is dedicated by the governing standards. For instance, in the IS-54 standard, $\gamma$ is $14 \mathrm{~dB}$ while in GSM, it is 9dB (as we discussed in chapter 3 ). 


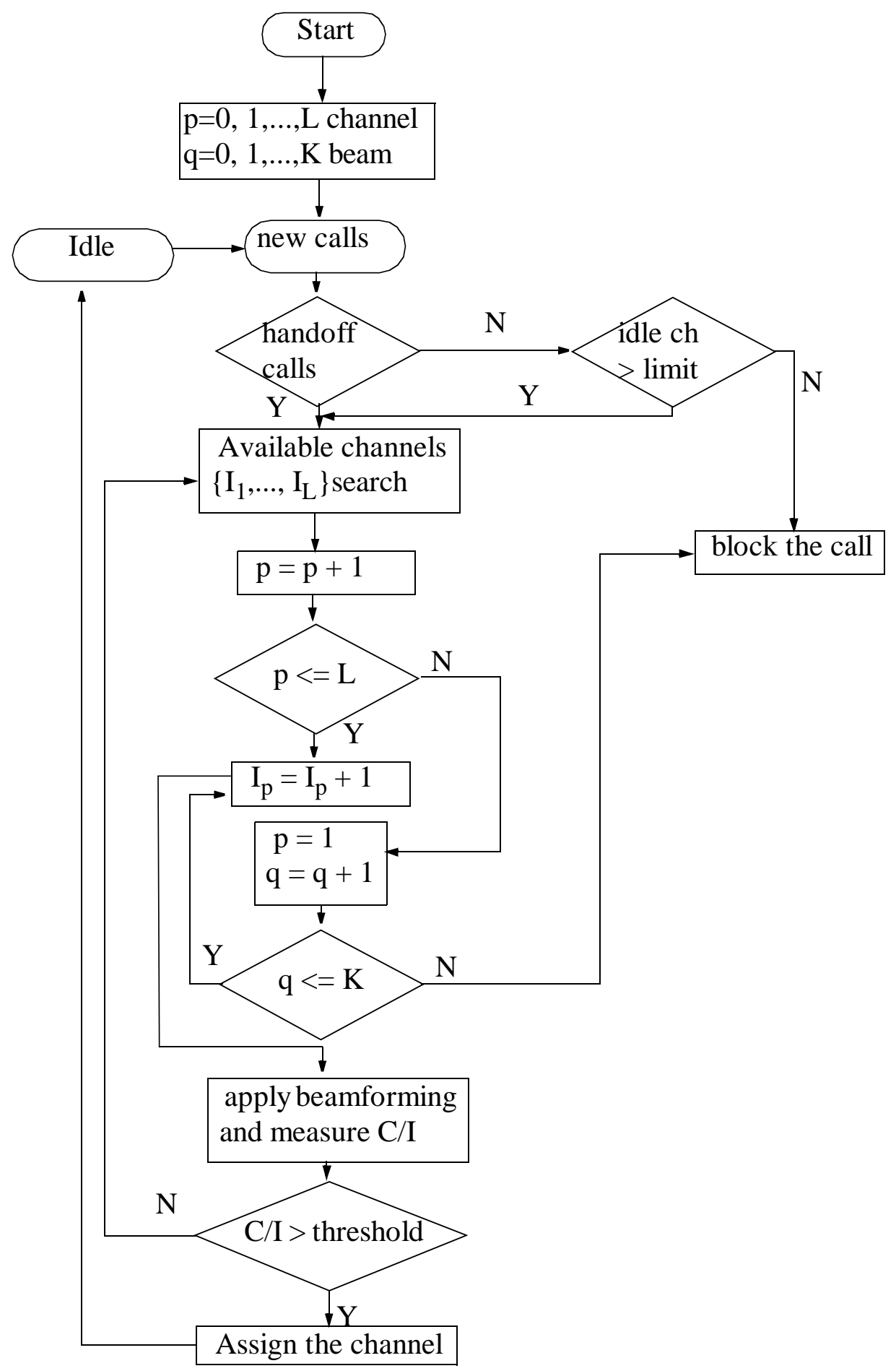

Figure 5.8 Flow chart of the channel assignment algorithm with smart antenna 


\section{Chapter 6}

\section{Methodology for complexity analysis of SWR architecture}

This chapter describes how the complexity of a software defined radio receiver with smart antenna implementation may be estimated in terms of the numbers of operations that a DSP processor must perform.

The resource estimation method in [4] has been extended to GSM system with smart antenna application.

\subsection{DSP complexity analysis method}

System complexity are calculated based on each individual function and its related demand of complex multiplication and additions required at each computation.

The calculation demand in each block can be parameterized in terms of the number of symbols per block, the channel length in chips, and the instantaneous number of users and can be related directly to the number of millions of operations per second (MOPS) needed to implement the complex multiply and addition operations. For the case using adaptive smart antennas, a simple multiplier of the number of antenna elements is proposed for an approximation. 


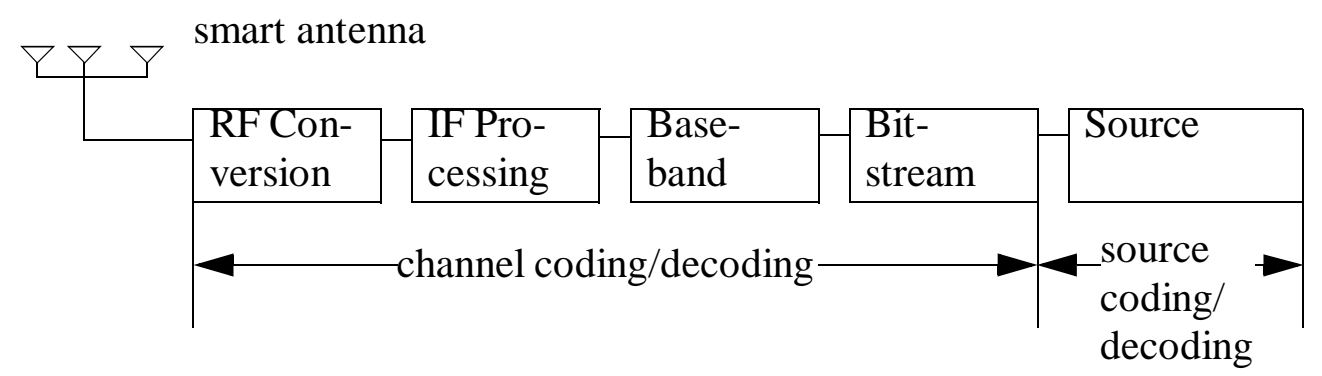

Figure 6.1: The canonical software radio function architecture with smart antenna application

\subsection{Partitioning of the channel processing stream}

The first step of processing complexity calculation is partitioning of the channel processing stream. The canonical software radio architecture partitions are given in Figure 6.1, including segments like antennas, RF conversion, IF processing, baseband processing and bitstream processing.

\subsubsection{Antenna segment}

Here we consider that smart antennas with multiple antenna elements are used in the software radio architecture (see Figure 6.1). Beamforming is used for user tracking and interference reduction.

The number of antenna elements depends on the demand of interference cancellation. Normally, beamforming with $\mathrm{N}$ antenna elements can place $\mathrm{N}-1$ adaptive nulls on 
interferers which are sufficiently separated in azimuth. However, more elements are needed if the multipath effects are considered.

The number of elements will directly affect the calculation demands of the baseband processing and part of the IF processing.

\subsubsection{RF conversion segment}

This segment includes output power generation, preamplification, and RF/IF conversion for suitable A/D/A conversion. In most radio bands, RF conversion will be analog. Certain critical RF problems are exacerbated in the software radio. These include the need for linearity in the amplifier, which is the critical problem in the RF conversion segment. The wider the access bandwidth, the higher requirements of linearity.

\subsubsection{IF processing segment}

The IF processing segment realizes the mapping between modulated baseband and IF signal. IF filtering recovers the medium channels (e.g., a $200 \mathrm{kHz}$ TDMA channel in GSM and/or a 30kHz TDMA channel in IS-54) and converts the signal to baseband. The complexity of this segment depends on the requirements of frequency conversion and filtering.

Typically, a $12.5 \mathrm{MHz}$ mobile cellular band is sampled at $30.72 \mathrm{MHz}$ with $\mathrm{N}$ samples per second. The frequency translation, filtering and decimation normally requires 100 operations per sample[4]. So the total processing demand will be more than 3000 MOPS.

For GSM, the access IF bandwidth $\mathrm{W}_{\mathrm{a}}=25 \mathrm{MHz}$, if the sample rate is 2.5 sample/ $\mathrm{Hz}$ and 200 operations are needed for each sample, the processing for $\mathrm{D}_{\text {if }}=25 \mathrm{MHz} * 2.5$ samples/Hz $* 200$ OPS/sample $=12500$ MOPS. 


\subsubsection{Baseband processing segment}

The baseband segment includes the channel modulation, predistortion for nonlinear channels and trellis coding and soft decision parameter estimation. The complexity of this segment depends on the bandwidth at baseband $\mathrm{W}_{\mathrm{c}}$, the complexity of the channel waveform and the complexity of related processing(e.g., soft decision support). For GSM, $\mathrm{W}_{\mathrm{c}}=200 \mathrm{kHz}$, if 50 operations are needed for each unit frequency per second per user, the processing for baseband $\mathrm{D}_{\mathrm{bb}}=200 \mathrm{kHz} * 50 \mathrm{OPS} / \mathrm{Hz} / \mathrm{User}=10 \mathrm{MOPS} /$ User.

\subsubsection{Bitstream segment}

The bitstream segment mainly includes multiplexing/demultiplexing, forward error control (FEC), including interleaving and convolutional coding, frame alignment, bit-stuffing, and radio link encryption. The complexity of this segment depends on multiplexing, framing, FEC, encryption, and related bit manipulation operations.

Demand increases linearly with the number of simultaneously active subscribers who share a beam channel using a time division scheme. For GSM, bit stream rate $\mathrm{R}_{\mathrm{b}}=$ $128 \mathrm{kbps}$, if 100 operations are needed for each bit per user, the processing for bitstream $\mathrm{D}_{\mathrm{bs}}=128 \mathrm{kbps} * 100 \mathrm{OPS} / \mathrm{bit} / \mathrm{User}=12.8 \mathrm{MOPS} /$ User.

\subsubsection{Source segment}

The source segment contains the source coding scheme. For GSM, it is RPE-LTP.

The signaling control processing is related to each segment. For GSM, 8MOPS processing demand per site is considered in this Chapter [4]. 


\subsection{Resource requirement estimation}

Resources critical to the software radio architecture include I/O bandwidth, memory and processing capacity. Good estimates of the demand for these resources result in a well informed mapping of the above segments to appropriate hardware.

\subsubsection{Estimate demand in the context of the canonical data flow}

Our demand estimation is based on the function partitioning in Figure 6.1.

Main parameters for demand estimation are given in Table 6.1. The demand estimation mainly depends on the signal bandwidths and on the complexity of key operations for IF, baseband, bitstream, and source segment processing. An approximation of the demand estimation is given as follows:

$$
\mathrm{D}=\mathrm{D}_{\mathrm{if}}+\mathrm{N}_{\mathrm{bn}} * \mathrm{~N}_{\mathrm{ts}} *\left(\mathrm{D}_{\mathrm{bb}}+\mathrm{D}_{\mathrm{bs}}+\mathrm{D}_{\mathrm{s}}\right)+\mathrm{D}_{0}
$$

where:

$\mathrm{D}_{\text {if }}$ stands for the IF processing demand which is proportional to the acquisition bandwidth $\left(\mathrm{W}_{\mathrm{a}}\right)$, and to the complexity of the IF band isolation filter.

$\mathrm{N}_{\mathrm{bn}}$ is the number of beams and $\mathrm{N}_{\mathrm{ts}}$ is the number of users to share one beam channel. $\mathrm{N}_{\mathrm{bn}} * \mathrm{~N}_{\mathrm{ts}}$ is then the maximum number of simultaneous subscribers to be served.

$\mathrm{D}_{\mathrm{bb}}$ is the baseband demand, proportional to the bandwidth of a single user channel $\left(\mathrm{W}_{\mathrm{c}}\right)$ and the complexity of demodulation.

$\mathrm{D}_{\mathrm{bs}}$ is the bitstream demand, proportional to the data rate $\left(\mathrm{R}_{\mathrm{b}}\right)$ and the complexity of the forward error control algorithm, bit stuffing/interleaving, signaling and control and miscellaneous operations functions. 
$\mathrm{D}_{\mathrm{s}}$ is the source segment demand.

$\mathrm{D}_{0}$ is the management overhead processing demand.

\subsection{Estimating total resource requirements}

The total resource requirements for GSM are estimated in Table 6.2. This table shows the relationship between the key parameters and approximation of processing demand. This approximation is used for the hardware design when implement the software radio architecture.

In this table, we follow the assumptions used in [4]. We consider 50OPS/Hz for baseband demodulator, 100 MOPS bit per second for bitstream signaling and FEC, 2MOPS per user for source coding and $8 \mathrm{MOPS} /$ site for SS7 signaling. The total DSP MOPS is about 1496 MOPS per cell site, excluding 12500 MOPS for IF processing which can be implemented in dedicated digital hardware separately.

Table 6.1: Critical parameters for demand estimation

\begin{tabular}{|c|c|c|c|c|c|c|c|c|c|}
\hline $\begin{array}{l}\text { Typical } \\
\text { Appli- } \\
\text { cation }\end{array}$ & $\begin{array}{l}\text { RF } \\
\text { fc }\end{array}$ & $\begin{array}{l}\mathrm{Wa} \\
\mathrm{MHz}\end{array}$ & $\begin{array}{l}\mathrm{IF} \\
\mathrm{W}_{\mathrm{c}} \\
\mathrm{Hz}\end{array}$ & $\begin{array}{l}\text { Chan- } \\
\text { nel } \\
\text { code }\end{array}$ & $\begin{array}{l}\text { Base- } \\
\text { band } \mathrm{W}_{\mathrm{i}} \\
\text { kbps }\end{array}$ & $\begin{array}{l}\text { Bitstream } \\
\text { States } \\
\text { FEC }\end{array}$ & $\begin{array}{l}\text { Multi- } \\
\text { plexing }\end{array}$ & Privacy & Source \\
\hline GSM & UHF & 25 & $200 \mathrm{k}$ & GMSK & 270.83 & $\mathrm{CPF}$ & TDM & Encrypt & RPE-LTP \\
\hline
\end{tabular}

Table 6.2: Approximation processing demand estimation: GSM with smart antenna

\begin{tabular}{|l|l|c|l|}
\hline Segment & Parameter & Illustrative value & Demand estimate \\
\hline \multirow{2}{*}{ IF } & $\mathrm{Wa}$ & $\begin{array}{c}25 \mathrm{MHz} \\
(2.5 \text { oversampling })\end{array}$ & \\
\cline { 2 - 3 } & IF Filter & $200 \mathrm{OPS} / \mathrm{Hz}$ & \\
\hline Users & $\mathrm{N}_{\mathrm{bn}}{ }^{*} \mathrm{~N}_{\mathrm{ts}}$ & $60 /$ cell site & \\
\hline \multirow{2}{*}{ Baseband } & Wc & $200 \mathrm{kHz}$ & \multirow{2}{*}{ Dbb $=10 \mathrm{MOPS} / \mathrm{user}$} \\
\cline { 2 - 3 } & Demodulator & $50 \mathrm{OPS} / \mathrm{Hz}$ & \\
\hline
\end{tabular}


Table 6.2: Approximation processing demand estimation: GSM with smart antenna

\begin{tabular}{|l|l|l|l|}
\hline \multirow{2}{*}{ Bitstream } & $\mathrm{Rb}$ & $128 \mathrm{kbps}$ & Dbs $=12.8 \mathrm{MOPS} / \mathrm{user}$ \\
\cline { 2 - 3 } & FEC, Signaling & $100 \mathrm{OPS} / \mathrm{b}$ & \\
\hline Source & RPE-LTP Codec & $2 \mathrm{MOPS} / \mathrm{user}$ & Ds $=2 \mathrm{MOPS} / \mathrm{user}$ \\
\hline Signaling & SS7 & $8 \mathrm{MOPS} /$ site & Do $=8 \mathrm{MOPS} / \mathrm{user}$ \\
\hline SUM & DSP MOPS & & $\begin{array}{l}\text { D }=1496 \mathrm{MOPS} \text { per cell } \\
\text { site, excluding Dif }\end{array}$ \\
\hline
\end{tabular}

\subsection{Limitations of the proposed method}

The proposed method of the complexity analysis are based on ideal assumptions such as the perfect patitioning of the system functions. However, the whole demand calculation is not that simple. On the top of the basic estimate for complex functions, an overhead must be added due to other factors such as non-ideal algorithm partitioning, less than $100 \%$ use of DSP resources and administration and control functions. 
Chapter 7

\section{Conclusions and Recommendations for Future Research}

\subsection{Summary and conclusion}

\subsubsection{Summary}

Chapter 1 introduces the research background of the two key solutions, the smart antenna and software radio, which are used for higher capacity, better quality and more flexibility supported in next-generation wireless systems.

A review of the concepts and brief survey of previous work in smart antennas and software radio are provided in Chapter 2. The synergy of the study of the two key technology applications are addressed.

In Chapter 3, downlink capacity is examined and analyzed for a frequency hopping GSM system with a multibeam smart antenna application in the base station. Comparison is given to the case of sectorization only and no power control. It is shown that a multibeam antenna combined with power control can dramatically enhance the GSM system capacity, particularly at low and medium loading values.

An extension work to the previous research of [24] is given in Chapter 4. Here, the parameterized design method is used and a complete TDMA software radio baseband receiver architecture is proposed to support a multi-standard system. 
In Chapter 5, a software radio base station architecture with smart antenna application is presented and analyzed. We see that the smart antenna can be implemented in the base station on top of the baseband architecture proposed in Chapter 4. Advantages and limitations are addressed.

Chapter 6 describes one possible methodology for complexity analysis of software radio architecture.

The summary, conclusion and suggestions for further research are included in Chapter 7.

\subsubsection{Conclusion}

Smart antennas are investigated and shown to be a powerful tool for increasing wireless network capacity of FH-TDMA cellular systems. A multiple beam antenna combined with power control can dramatically enhance the system capacity particularly at low and medium loading values. This may serve as an upper bound which can be approached in reality as technology for perfect power control is improving.

One software radio base station architecture with smart antenna is proposed and serves as a good example to illustrate the great flexibility provided by the software radio solution. In this architecture, the smart antenna algorithms can be dynamically reconfigured according to different environment requirements and the baseband processing can also be dynamically reconfigured according to different standards requirements.

The whole research work will be presented on ICC2000. 


\subsection{Recommendations for future research}

As one of the further extensions, DSP and FPGA implementation of the proposed software radio architecture will be addressed in more detail for FH-TDMA.

The above research work can be further extended to third generation system such as UMTS, considering the multistandard convergency.

Not only the smart antennas, but also the algorithms to implement adaptive channel allocation, adaptive power control and bandwidth adaptation, can be incorporated efficiently in the existing base station with the flexibility provided by software radio architecture. However, the detailed design for the integration of those algorithms needs more study.

There are still many problems in the development of software radio. The demand of high quality wideband RF access and high performance analog to digital and digital to analog conversion is high. Very linear analog amplifers and mixers must be developed to meet the large dynamic range requirements in multi-band, multimode receivers. Especially when integrated with a smart antenna system, the complexity of the RF stage of software radio is increased and a more intelligent transceiver architecture is needed.

There are still many hardware and software implementation issues which need more study, such as how the complexity of the algorithm operations will affect the hardware implementation efficiency and the time domain or frequency domain of the algorithms will affect the software implementation efficiency.

Also, it is still difficult to find suitable methods to estimate the signal processing requirement and to estimate the processing capacity of the common platform which consists of multiple DSP processors, CPUs, memory modules amd low/high speed busses, if several systems are implemented in one common platform. 


\section{References}

[1] Wei Wang, Mohamed Ahmed,Samy Mahmoud, Roshdy H.M. Hafez, "Downlink Capacity Enhancement in GSM System Using Multiple Beams Smart Antenna and SWR Implementation," accepted by the 10th Annual Virginia Tech Symposium on Wireless Personal Communications and published in the MPRG proceedings and in a book being prepared for Kluwer Academic Press in June 2000.

[2] Mohamed Ahmed, Wei Wang, Samy Mahmoud, "Downlink Capacity Enhancement in GSM Systems with Frequency Hopping and Multiple Beam Smart Antennas," accepted by ICC2000 and publicated in the Proceedings of the conference in June 2000.

[3] Mohamed Ahmed and Samy Mahmoud, "Capacity Analysis of GSM Systems using Frequency Hopping and Smart Antennas," to be Published in IEEE VTC2000, vol.1, pp.355-359, Tokyo, Japan, May 2000.

[4] J. Mitola III, “The Software Radio Architecture," IEEE Communications Magazine, pp.26-38, May 1995.

[5] J. Mitola III, "Software Radio Architecture: A Mathematical Perspective," IEEE JSAC, vol. 17, No. 4, pp.514-538 April 1999.

[6] P. M. Athanas, J. H. Reed, and W. H. Tranter, "A Prototype Software Radio Based on Configurable Computing,” Advancing Microelect, pp.34-39, 1998.

[7] H. Tsurumi and Y. Suzuki, "Broadband RF Stage Architecture for Software-Defined Radio in Handheld Terminal Applications," IEEE Communcation Magazine, pp.9095, Feb. 1999.

[8] P. M. Athanas et al., "A High Capacity Adaptive Wireless Receiver Implemented with a Reconfigurable Computer Architecture," ARPA GloMo Principle Investigators Conference, San Diego, CA, Nov. 1995.

[9] P. Leppanen et al., "Software Radio -- An Alternative for the Future in Wireless Personal and Multimedia Communications," IEEE International Conference Personal Wireless Communcation, Jaipur, India, pp.364-68, Feb. 1999.

[10] S. Srikanteswara et al., "Configurable Computing for Communication Systems," Proceedings of Wireless Communication Conference, IMAPS, pp.180-85, 1998.

[11] R. Bittner, "Wormhole Run-Time Reconfiguration: Conceptualization and VLSI Design of a High Performance Computing System," Ph.D. dissertation, Department of Electrical and Computer Engineering, Virginia Tech, 1997. 
[12] J. Mitola III, "Software Radio Architecture and Technology," Proceedings 1998 International Symposium Adv. Radio Tech., Boulder, CO, Sept. 1998.

[13] J. Pereira, "Beyond Software Radio, Towards Reconfigurability Across the Whole System and Across Networks," Proceedings VTC Fall '99, Amsterdam, vol.5 pp.2815-2818 Sept. 1999.

[14] R. Kohno, "Spatial and Temporal Communication Theory Using Adaptive Antenna Array,” IEEE Personal Communications, pp.28-35, Feb. 1998.

[15] H. Dam et al., "Performance Evaluation of Adaptive Antenna Base Station in a Commercial GSM Network," Proceedings VTC Fall '99, Amsterdam, vol.1 pp.47-51, Sept. 1999.

[16] S. Gordon, M. Feuerstein, and M. Zhao, "Methods for Measuring and Optimizing Capacity in CDMA Networks Using Smart Antennas," Proceedings 9th VA Tech/ MPRG Symposium, Blacksburg, VA.

[17] G. Tsoulos, M. Beach, and S. Swales, "Adaptive Antennas for Third Generation DSCDMA Cellular Systems," in Proceedings 9th ICAP, Eindhoven, the Netherlands, vol.1, April 1995, pp.137-140.

[18] B. Adrian and S. Haggman, "UMTS Radio Network Simulation with Smart Antennas," Proceedings 9th VA Tech/MPRG Symposium., Blacksburg, VA.

[19] LAL C. Godara, “Applications of Antenna Arrays to Mobile Communications, Part I: Performance Improvement, Feasibility, and System Considerations," Proceedings of the IEEE. vol.85, No.7, pp.1029-1030, July 1997

[20] LAL C. Godara, “Applications of Antenna Arrays to Mobile Communications, Part II: Beam-Forming and Direction-of_arrival Considerations," Proceedings of the IEEE. vol.85, No.7, pp.1031-1060, July 1997

[21] G. Xue et al., “Adaptive Multistage Parallel Interference Cancellation for CDMA over Multipath Fading Channels," Proceedings IEEE VTC '99, Houston, TX, pp. 1815-1827, May 1999.

[22] C. Wijting et al., "Groupwise Serial Multi-User Detectors for Multirate DS-CDMA," Proceedings IEEE VTC '99, Houston, TX, pp.836-840, 1999.

[23] A. Paulraj, "Smart Antennas in Wireless Communications," Proceedings 5th Annual Workshop. Smart Antennas in Wireless Mobile Communications, Stanford Univ./ SARG, July 1998.

[24] T. Turletti and D. Tennenhouse, "Complexity of Software GSM Base Station," IEEE Communications Magazine, pp.113-117, Feb. 1999. 
[25] S. Chuprun et al., "High Data Rate OFDM Waveform Capabilities of Emerging Software Defined Radio Platforms," Proceedings IEEE MILCOM '99, Atlantic City, NJ, Nov. 1999.

[26] P. Leppanen, J. Reinila, "Software radio-an alternative for the future in wireless personal and multimedia," Personal Wireless Communication, 1999 IEEE International Conference, pp.364-368, 1999

[27] S. Kourtis, P. McAndrew, P. Tottle, "Software radio 2G \& 3G inner receiver processing," UMTS Terminals and Software Radio (Ref. No. 1999/055), Colloquium, pp. 6/1-6/7, 1999.

[28] Li Weidong, Yao Yan, "Software radio: technology and implementation," International Conference on Communication Technology Proceedings, ICCT '98, vol.1, pp. $5,1998$.

[29] W. Tuttlebee, "Software radio-impacts and implications," Spread Spectrum Techniques and Applications, Proceedings., IEEE 5th International Symposium, pp.541545 , vol.2, 1998.

[30] K.C. Zangi, R.D. Koilpillai, "Software radio issues in cellular base stations," Selected Areas in Communications, IEEE Journal vol.17, no.4, pp.561-573, April 1999.

[31] W.H.W. Tuttlebee, "Software radio technology: a European perspective," IEEE Communications Magazine, vol. 37, no. 2, pp.118-123, Feb. 1999.

[32] J. Mitola, "The software radio architecture," IEEE Communications Magazine, vol. 33, no. 5, pp.26-38, May 1995.

[33] C. Noblet, A.H. Aghvami, "Assessing the over-the-air software download for reconfigurable terminal," Personal Communications in the 21st Century (II) (Ref. No.1998/242), IEE Colloquium, pp.6/1-6/6, 1998.

[34] Ogose, S. "Application of software radio to the third generation mobile telecommunications," Vehicular Technology Conference IEEE 49th, vol.2, pp.1212-1216, 1999.

[35] L.III Mitola, "Technical challenges in the globalization of software radio," IEEE Communications Magazine, vol.37, no.2, pp.84-89, Feb. 1999.

[36] J. Kennedy, M.C. Sullivan, "Direction finding and smart antennas using software radio architectures," IEEE Communications Magazine, vol.33, no.5, pp.62-68, May 1995.

[37] A.Wiesler, R.Machauer, F. Jondral, "Comparison of GMSK and linear approximated GMSK for use in software radio," Spread Spectrum Techniques and Applications, 1998. Proceedings., 1998 IEEE 5th International Symposium, vol.2, pp.557560,1998 . 
[38] G.C. Ahlquist, M. Rice, B. Nelson, "Error control coding in software radios: an FPGA approach," IEEE Personal Communications vol.6, no.4, pp.35-39, Aug. 1999.

[39] W.H.W. Tuttlebee, "Software-defined radio: facets of a developing technology," IEEE Personal Communications, vol.6, no.2, pp.38-44, Aug. 1999.

[40] M. Cummings, S. Haruyama, "FPGA in the software radio," IEEE Communications Magazine, vol.37, no.2, pp.108-112, Feb. 1999.

[41] H.R. Karimi, B. Friedrichs, "Wideband digital receivers for multi-standard software radios," Adaptable and Multistandard Mobile Radio Terminals (Ref. No. 1998/406), IEE Colloquium, pp.5/1-5/7, 1998.

[42] A.S. Margulies, J. III Mitola, "Software defined radios: a technical challenge and a migration strategy," Spread Spectrum Techniques and Applications, 1998. Proceedings., 1998 IEEE 5th International Symposium, vol. 2, pp.551-556, 1998.

[43] A.Wiesler, F. Jondral, "Software radio structure for second generation mobile communication systems," Vehicular Technology Conference, 1998. VTC 98. 48th, vol. 3, pp.2363-2367, 1998.

[44] H. Erben, K. Sabatakakis, "Advanced software radio architecture for 3rd generation mobile systems," Vehicular Technology Conference, 1998. VTC 98. 48th IEEE, vol. 2, pp.825-829, 1998.

[45] R. Kohno, "Software antenna and its communication theory for mobile radio communications," Personal Wireless Communications, 1997 IEEE International Conference, pp.227-233, 1997.

[46] T. Turletti, D. Tennenhouse, "Complexity of a software GSM base station," IEEE Communications Magazine, vol.37, no.2, pp.113-117, Feb. 1999.

[47] F. Riera-Palou, C. Chaikalis, J.M. Noras, "Reconfigurable mobile terminal requirements for third generation applications ", UMTS Terminals and Software Radio (Ref. No. 1999/055), IEE Colloquium, pp. 9/1-9/6, 1999.

[48] A.Wiesler, H. Schober, R. Machauer, F. Jondral, "Software radio structure for umts and second generation mobile communication systems," Vehicular Technology Conference, 1999. VTC 1999 - Fall. IEEE VTS 50th, vol. 2, pp.939-942, 1999.

[49] J. Razavilar, F. Rashid-Farrokhi, K.J.R. Liu, "Software radio architecture with smart antennas: a tutorial on algorithms and complexity," Selected Areas in Communications, IEEE Journal, vol.17, no.4, pp.662-676, April 1999.

[50] J. Kennedy, M.C. Sullivan, "Direction finding and smart antennas using software radio architectures," IEEE Communications Magazine, vol.33, no.5, pp.62-68, May 1995. 
[51] Shihe Li, "Key technologies in SCDMA wireless access," Microwave Conference Proceedings, 1997. APMC '97, Asia-Pacific, vol.1, pp.169-172, 1997.

[52] R. Kohno, "Spatial and temporal communication theory using adaptive antenna array ," IEEE Personal Communications, vol.5, no.1, pp.28-35, Feb. 1998.

[53] R. Kohno, "Software antenna and its communication theory for mobile radio communications," Personal Wireless Communications, 1997 IEEE International Conference, pp.227-233, 1997.

[54] J.-J. Monot, J. Thibault, P. Chevalier, F. Pipon, S. Mayrargue, A. Levy, "A fully programmable prototype for the experimentation of the SDMA concept and use of smart antennas for UMTS and GSM/DCS1800 networks," Personal, Indoor and Mobile Radio Communications, 1997. Waves of the Year 2000. PIMRC '97., The 8th IEEE International Symposium, vol. 2, pp.534-538, 1997.

[55] R.Comitangelo, D. Minervini, B.Piovano, "Beam forming networks of optimum size and compactness for multibeam antennas at $900 \mathrm{MHz}$ " Antennas and Propagation Society International Symposium, 1997. IEEE., 1997 Digest, vol. 4 , pp.2127-2130, 1997.

[56] Y.S. Song, H.M. Kwon, "Analysis of a simple smart antenna for CDMA wireless communications," Vehicular Technology Conference, 1999 IEEE, vol. 1, pp.254$258,1999$.

[57] S. S. Haykin, “Adaptive Filter Theory,” Englewood Cliffs, NJ: Prentice-Hall, 1991

[58] Adrian Boukalov, Sven-Gustav Haggman, "System aspects of smart antennas technology in cellular wireless communications, an overview," Wireless Communications, 1999

[59] “Multifunction phased array," http://www.i-a-i.com/projects/array/

[60] K. Murota and K. Hirade, "GMSK modulation for digital mobile radio telephony," IEEE Transaction Communications, 29(7):1044-1050, July 1981.

[61] P.A. Larurent. "Exact and approximate construction of digital phase modulation by superposition of amplitud modulated pulses," IEEE Vehicular Technology Conference, COM-34: 150-160, February 1986.

[62] P.Jung. "Laurent's representation of binary digital continuous phase modulated signals with modulation index 1/2 revisited," IEEE Transaction Communications, 42:221-224, 1994.

[63] GSM 05.05 (ETS 300 577). Radio transmission and reception. ETSI. 